\title{
Tumor cell survival pathways activated by photodynamic therapy: a molecular basis for pharmacological inhibition strategies
}

\author{
Mans Broekgaarden ${ }^{1}$ Ruud Weijer ${ }^{1}$ - Thomas M. van Gulik ${ }^{1}$. \\ Michael R. Hamblin ${ }^{2,3,4}$. Michal Heger ${ }^{1}$
}

Published online: 29 October 2015

(C) The Author(s) 2015. This article is published with open access at Springerlink.com

tumor microenvironment in favor of tumor survival. In this review, the molecular mechanisms are elucidated that occur post-PDT to mediate cancer cell survival, on the basis of which pharmacological interventions are proposed. Specifically, pharmaceutical inhibitors of the molecular regulators of each survival pathway are addressed. The ultimate aim is to facilitate the development of adjuvant intervention strategies to improve PDT efficacy in recalcitrant solid tumors.

Keywords Apoptosis signaling kinase $1 \cdot$ Heat shock factor 1 . ER stress $\cdot$ Antioxidant response $\cdot$ Inflammatory response $\cdot$ Proteotoxic stress nism, helps cancer cells to cope with the PDT-induced oxidative stress and cell damage. These survival pathways are mediated by the transcription factors activator protein 1 (AP-1), nuclear factor E2-related factor 2 (NRF2), hypoxia-inducible factor 1 (HIF-1), nuclear factor $\mathrm{kB}$ $(\mathrm{NF}-\kappa \mathrm{B})$, and those that mediate the proteotoxic stress response. The survival pathways are believed to render some types of cancer recalcitrant to PDT and alter the

Michal Heger

m.heger@amc.uva.nl

Department of Experimental Surgery, Academic Medical Center, University of Amsterdam, Meibergdreef 9, 1105

AZ Amsterdam, The Netherlands

$\begin{array}{ll}\text { Abbreviations } \\ \text { 2-ME } & \text { 2-Methoxyestradiol } \\ \text { 3-AT } & \text { 3-Amino-1,2,4-triazole } \\ \text { 4EBP1 } & \text { 4E-binding protein 1 } \\ \text { ABCG2 } & \text { ATP-binding cassette subfamily G member 2 } \\ \text { ABL } & \text { Abelson murine leukemia viral oncogene } \\ \text { AK } & \text { Actinic keratoses } \\ \text { ALA } & \text { Aminolevulinic acid } \\ \text { AP-1 } & \text { Activator protein 1 } \\ \text { APAF1 } & \text { Apoptotic protease activating factor 1 } \\ \text { APO } & \text { Angiopoietin } \\ \text { ARE } & \text { Antioxidant response element } \\ \text { ARNT } & \text { Aryl hydrocarbon receptor nuclear } \\ & \text { translocator } \\ \text { ASK1 } & \text { Apoptosis signal-regulating kinase 1 } \\ \text { ATF } & \text { Activating transcription factor } \\ \text { PKD } & \text { Protein kinase D } \\ \text { BAX } & \text { BCL2-associated X protein } \\ \text { BAK } & \text { BCL2 homologous antagonist killer } \\ \text { BC } & \text { Bladder cancers } \\ \text { BCL } & \text { B-cell lymphoma protein }\end{array}$




\begin{tabular}{|c|c|c|c|}
\hline $\mathrm{BCR}$ & Breakpoint cluster region protein & GLUT & Glucose transporter \\
\hline bHLH & Basic helix-loop-helix & GM-CSF & Granulocyte-macrophage colony stimulating \\
\hline BID & $\mathrm{BH} 3$ interacting domain death agonist & & factor \\
\hline BIP & Binding immunoglobulin protein & gp130 & Glycoprotein 130 \\
\hline \multirow[t]{2}{*}{ BIRC } & Baculoviral inhibitor of apoptosis repeat- & GRP78 & Glucose-regulated protein 78 \\
\hline & containing & GST & Glutathione S-transferase \\
\hline \multirow[t]{2}{*}{ BNIP3 } & BCL2/adenovirus E1B 19 kDa protein- & HBEGF & Heparin-binding EGF \\
\hline & interacting protein 3 & HIF-1 & Hypoxia-inducible factor 1 \\
\hline BNIP3L & BNIP3 ligand & $\mathrm{HK}$ & Hexokinase \\
\hline $\mathrm{BSO}$ & L-Buthionine sulfoximine & $\mathrm{HO}-1$ & Heme oxygenase- 1 \\
\hline bZIP & Basic leucine zipper domain & HRE & Hypoxia responsive element \\
\hline $\mathrm{C} / \mathrm{EBP}$ & CCAAT-enhancer binding protein & HSF1 & Heat shock factor 1 \\
\hline CBP & CREB binding protein & HSP70 & Heat shock protein 70 \\
\hline CCR7 & C-C chemokine receptor 7 & HSP90 & Heat shock protein 90 \\
\hline CCL & Chemokine $\mathrm{C}-\mathrm{C}$ motif ligand & ICAM & Intracellular adhesion molecule \\
\hline CD40L & Cluster of differentiation 40 ligand & IFN & Interferon \\
\hline $\mathrm{CDC}$ & Cell division cycle protein & IGF & Insulin-like growth factor \\
\hline CES1A1 & Carboxylesterase 1A1 & IL & Interleukin \\
\hline \multirow[t]{2}{*}{ CFLAR } & Caspase 8 and frequently associated with death & IL-6R & IL-6 receptor \\
\hline & domain-like apoptosis regulator & IкB & Inhibitor of $\mathrm{\kappa B}$ \\
\hline CHOP & C/EBP homologous protein & IKK & IкB kinase \\
\hline cIAP & Cytosolic inhibitor of apoptosis protein & IRE1 & Inositol-requiring enzyme 1 \\
\hline $\mathrm{CO}$ & Carbon monoxide & JNK & c-Jun N-terminal kinase \\
\hline $\mathrm{COX}$ & Cyclooxygenase & KEAP1 & Kelch-like ECH-associated protein 1 \\
\hline CREB & Cyclic AMP response element & KGF & Keratinocyte growth factor \\
\hline CRT & Calreticulin & KRIBB & $N^{2}$-(1H-indazole-5-yl)- $N^{6}$-methyl-3- \\
\hline CXCL & Chemokine $\mathrm{C}-\mathrm{X}-\mathrm{C}$ motif ligand & & nitropyridine-2,6-diamine \\
\hline DCF & 2'-7'-Dichlorofluorescein & KRP1 & Kelch-related protein 1 \\
\hline $\mathrm{DCFH}_{2}$ & 2'-7'-Dichlorodihydrofluorescein & LDHA & Lactate dehydrogenase A \\
\hline DDC & Diethyl-dithiocarbamate & LUBAC & Linear ubiquitin assembly chain \\
\hline DHMEQ & Dehydroxymethylepoxyquinomicin & MAF & Musculoaponeurotic fibrosarcoma oncogene \\
\hline ECLC & Early central stage lung cancers & & homologue \\
\hline EDN1 & Endothelin 1 & MAPK & Mitogen-activated protein kinase \\
\hline EGFR & Epidermal growth factor receptor & MCL-1 & Myeloid leukemia cell differentiation protein \\
\hline EH-1 & Microscomal epoxide hydroxylase & MCT & Monocarboxylate transporter \\
\hline EIF & Eukaryotic translation initiation factor & MEF2 & Myocyte-specific enhancer factor \\
\hline ELK-1 & ETS domain containing protein & MK & MAPK-activated protein kinase \\
\hline EM & Esophageal malignancies & MKK & MAPK kinase \\
\hline EPO & Erythropoietin & MMP & Matrix metalloproteinase \\
\hline ERAD & ER-associated degradation & MNK & MAPK interacting kinase \\
\hline ERK & Extracellular signal regulated kinase & MRP & Multidrug resistance protein \\
\hline EZR & Ezrin & MSK & Mitogen- and stress-activated protein kinase \\
\hline FADD & FAS-associated with death domain & MTS1 & Methionyl-tRNA synthetase 1 \\
\hline FASR & FAS receptor & $\mathrm{NC}$ & Nasopharyngeal carcinoma \\
\hline FGF & Fibroblast growth factor & NEMO & NF- $\kappa \mathrm{B}$ essential modulator \\
\hline FIH & Factor inhibiting HIF-1 & $\mathrm{NF}-\kappa \mathrm{B}$ & Nuclear factor $\kappa \mathrm{B}$ \\
\hline FOS & FBJ murine osteosarcoma viral oncogene & NIK & NF- $\kappa B$ inducing kinase \\
\hline & homologue & NOS2 & (Inducible) nitric oxide synthase 2 \\
\hline FRA & FOS-related antigen & NQO1 & NAD(P)H:quinine oxidoreductase 1 \\
\hline FRP & Ferritin repressor protein & NRF2 & Nuclear factor E2-related factor 2 \\
\hline GADD & Growth arrested and DNA damage induced & NSAIDs & Nonsteroidal anti-inflammatory drugs \\
\hline & protein & ODD & Oxygen-dependent degradation domain \\
\hline GCL & Glutamate-cysteine ligase & PAI1 & Plasminogen activator inhibitor 1 \\
\hline
\end{tabular}




\begin{tabular}{ll} 
PERK & Protein kinase RNA-like ER kinase \\
PDH & Pyruvate dehydrogenase \\
PDT & Photodynamic therapy \\
PDGFRA & Platelet-derived growth factor receptor A \\
PDI & Protein disulfide isomerase \\
PFKL & Phosphofructokinase L \\
PG & Prostaglandin \\
PGK1 & Phosphoglycerate kinase 1 \\
PHD & Prolyl hydroxylase domain protein \\
PI3K & Phosphatidyl inositol 3 kinase \\
PKC & Protein kinase C \\
PKM & Pyruvate kinase (muscle) \\
PPIX & Protoporphyrin IX \\
PTGS2 & Prostaglandin synthase 2 \\
RAR $\alpha$ & Retinoic acid receptor $\alpha$ \\
RAS & Rat sarcoma protein \\
REL & Reticuloendotheliosis \\
RIPK & Receptor-interacting serine/threonine protein \\
& kinase \\
ROS & Reactive oxygen species \\
SC & Skin cancers \\
Ser & Serine \\
sgp130 & Soluble gp130 \\
SHIP & SH2-containing inositol 5'-phosphatase \\
sIL-6R & Soluble IL-6R \\
SIR2 & Sirtuin \\
SMAC & Second mitochondria-derived activator of \\
& caspases \\
SnPP & Tin PPIX \\
SOD & Superoxide dismutase \\
SRC & Sarcoma protein \\
SRXN1 & Sulfiredoxin \\
STAT3 & Signal transducer and activator of transcription 3 \\
TAK1 & Transforming growth factor $\beta$-activated kinase 1 \\
TBA 2 & Thromboxane A \\
TGF- $\beta$ & Transforming growth factor $\beta$ \\
Thr & Threonine \\
TNF- $\alpha$ & Tumor necrosis factor $\alpha$ \\
TNFR & TNF receptor \\
TPM & Tropomyosin \\
TRADD & TNFR associated with death domain \\
TRAF & Tumor necrosis factor receptor associated factor \\
TRAIL & TNF-related apoptosis-inducing ligand \\
TRX & Thioredoxin \\
uPA & Urokinase-type plasminogen activator \\
uPAR & uPA receptor \\
UPR & Unfolded protein response \\
VCAM & Vascular cell adhesion molecule \\
VEGF & Vascular endothelial growth factor \\
VHL & Von Hippel-Lindau protein \\
XBP1 & X-box binding protein 1 \\
XIAP & X-linked inhibitor of apoptosis \\
ZnPP & Zinc PPIX \\
& \\
\hline
\end{tabular}

\section{Introduction}

The standard treatments for solid tumors include surgery, chemotherapy, and/or radiotherapy. However, these treatments are often associated with high morbidity and are often unsuccessful. Consequently, alternative modalities must be devised to treat solid tumors with equal or improved clinical outcomes but in a more patient-friendly manner. Photodynamic therapy (PDT) is an alternative treatment modality that entails the systemic or topical administration of a photosensitizing agent followed by local irradiation of the photosensitizer-loaded tumor tissue with light of the appropriate wavelength to match the photosensitizer absorption. Irradiation causes the photosensitizer to first enter a short-lived excited singlet state that can transition to a long-lived excited triplet state [1]. Triplet state photosensitizers can transfer energy to molecular oxygen to yield singlet oxygen $\left({ }^{1} \mathrm{O}_{2}\right)$ by electron transfer electrons to form superoxide anion $\left(\mathrm{O}_{2}{ }^{\circ}\right)$ and hydroxyl radicals $\left(\mathrm{HO}^{\circ}\right)$. These reactive oxygen species (ROS) and their derivatives (such as lipid peroxides) subsequently oxidize biomolecules in the photosensitized tissue, causing cellular oxidative stress, tissue anoxia and tumor starvation due to ROS-mediated shutdown of tumor vasculature, and an antitumor immune response. Collectively these events contribute to cellular demise and removal of the tumor [2]. PDT provides important benefits compared to surgery, radiotherapy, and chemotherapy in that it is minimally invasive or even noninvasive and can be performed locally causing only minor damage to healthy tissue [3-5]. Moreover, PDT has been associated with increased life expectancy in cancer patients [6], is cost-effective $[4,7,8]$, generally does not require extended therapeutic follow-ups, and can easily be repeated in case of cancer recurrence. The latter is often difficult or impossible with the conventional therapies.

PDT has proven to be highly effective in the treatment of various types of cancer (Fig. 1a) [9-11, 13]. However, bladder and nasopharyngeal tumors exhibit poor complete response rates following PDT (Fig. 1a) [14-16]. For a variety of esophageal lesions and early-stage central lung cancers, the results differ greatly depending on the center administering the treatment and the exact type of PDT procedure performed [10,11]. With respect to the treatment of nonresectable extrahepatic cholangiocarcinomas, PDT has shown promising results by considerably improving the median survival of patients (Fig. 1b) [12], but the therapy is currently palliative and not curative.

The therapeutic failure in some of these cancer types likely stems from the use of photosensitizers with suboptimal optical and biochemical properties, inferior photosensitizer pharmacokinetics and/or pharmacodynamics, and variations in the tumor phenotype and genotype, which may positively influence tumor cell survival following PDT-induced oxidative damage [17]. While many investigators are looking at improving or developing new PDT strategies using chemistry or 

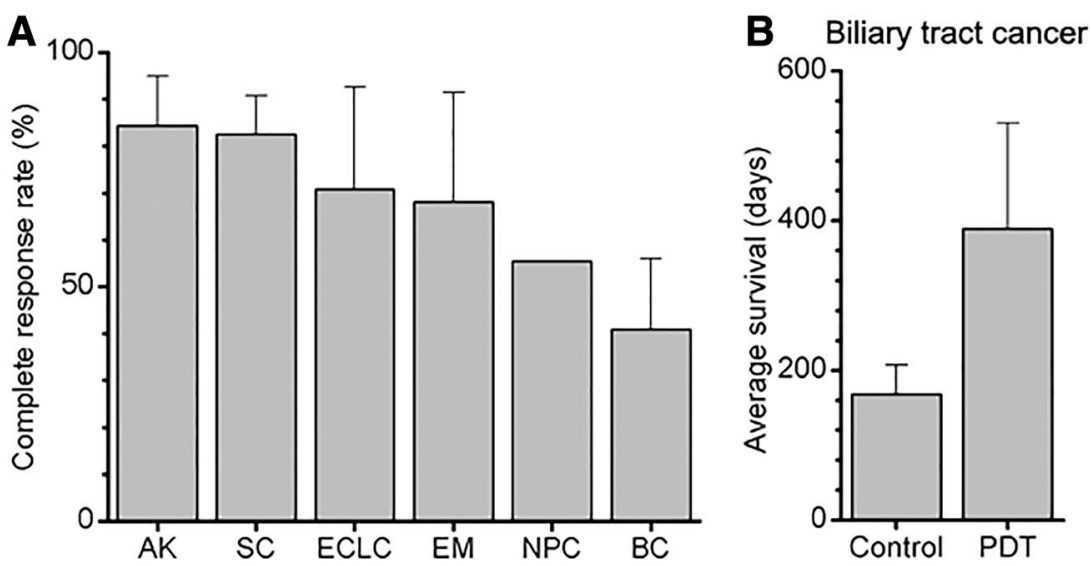

Fig. 1 a Overview of clinically obtained complete response rates with PDT of actinic keratoses $(A K)$, skin cancers $(S C)$, early stage central lung cancers $(E C L C)$, esophageal malignancies $(E M)$, nasopharyngeal carcinoma $(N P C)$, and bladder cancer $(B C)$. SC included (nodular) basal cell carcinomas and squamous cell carcinomas [9]. EM included Barrett's esophagus, low-grade dysplasia, high-grade dysplasia, and esophageal cancer [10]. BC included carcinoma in situ, recurrent superficial bladder cancer, and early stage lesions [11]. Complete response rates were averaged using the longest time interval in each study. b Average of the median survival time postdiagnosis of extrahepatic cholangiocarcinoma patients treated with PDT or left untreated (control) [12]. Adjuvant treatments, type of photosensitizer, light source, and light dose were not taken into account, as a result of which no statistical analyses were performed engineering approaches, relatively little research has been performed on the biology behind the therapeutic resistance, including the survival mechanisms that are triggered in cells to cope with the consequences of PDT. Several transcription factors have been identified that mediate cell survival following PDT (or approaches with similarities to PDT such as ultraviolet light irradiation). These include the members of the activating protein 1 (AP-1) transcription factor family, nuclear factor E2-related factor 2 (NRF2), hypoxia-inducible factor 1 (HIF-1), nuclear factor $\mathrm{\kappa B}(\mathrm{NF}-\mathrm{kB})$, heat shock factor 1 (HSF1), and transcription factors associated with the unfolded protein response (UPR).

In this review, a complete overview is provided of these pathways in terms of the activation mechanism, downstream biochemical and (patho)physiological effects, current state of knowledge regarding the involvement of these pathways in promoting tumor cell survival before and after PDT, as well as potential inhibition strategies for these pathways that can be used to increase the therapeutic efficacy of PDT.

\section{Photodynamic and biochemical activation of survival pathways}

\subsection{ROS production through photosensitizer excitation}

PDT encompasses laser or light irradiation of the tumorlocalized photosensitizer at a wavelength that corresponds to the photosensitizer's main absorption peak in the longer wavelength range of the visible spectrum (typically red light that is able to deeply penetrate tissue). Irradiation of a photosensitizer with light of a resonant frequency leads to photon absorption by the photosensitizer, resulting in the transition of an electron from the ground state $\left(\mathrm{S}_{0}\right)$ to an energetically higher but unstable first excited state $\left(\mathrm{S}_{1}\right)$ [18]. In most molecules, the $S_{1}$ electron rapidly (typically in the order of a few nanoseconds) undergoes vibrational relaxation and, in some instances, molecular relaxation during its decay to $S_{0}[18]$, producing heat and emission of a photon (fluorescence), respectively. However, $\mathrm{S}_{1}$ electrons in photosensitizers generally exhibit a strong tendency to undergo intersystem crossing, in which the energy of the photon is redistributed over two unpaired electrons with the same spin orientation. From this lower energy yet longer lived triplet $\left(\mathrm{T}_{1}\right)$ state, electrons can react with molecular oxygen $\left(\mathrm{O}_{2}\right)$ in their decay to $\mathrm{S}_{0}$. Two types of photochemical reactions can proceed from the $T_{1}$ state: type I reactions are characterized by electron transfer from the photosensitizer to $\mathrm{O}_{2}$, yielding $\mathrm{O}_{2}{ }^{--}[18-20] . \mathrm{O}_{2}{ }^{--}$has a relatively low reactivity but a long lifetime (several seconds) [21] and mainly acts as a precursor radical from which secondary and tertiary radicals are formed in biological systems [22]. Type II reactions are the result of energy transfer from the $\mathrm{T}_{1}$ electrons to $\mathrm{O}_{2}$, resulting in the production of highly reactive ${ }^{1} \mathrm{O}_{2}[18,23]$. The strong reactivity of ${ }^{1} \mathrm{O}_{2}$ toward lipids, nucleic acids, proteins, and other biochemical substrates is reflected by its short biological half-life $\left(3 \times 10^{-9} \mathrm{~s}\right)$ and the small area of effect in viable cells $\left(2-4 \times \times^{\circ} 10^{-6} \mathrm{~cm}^{2}\right)$ [24]. Additionally, since the ground state of $\mathrm{O}_{2}$ is the triplet state, only a minor amount of energy $\left(94.5 \mathrm{~kJ} \mathrm{~mol}^{-1}\right)$ is required for excitation to the singlet state, equivalent to the energy of a photon with a wavelength of $850 \mathrm{~nm}$ or shorter [18]. 


\subsection{Mechanisms of cytotoxicity}

\subsubsection{PDT-induced oxidative stress}

The production of ROS occurs during irradiation of the photosensitizer. Although these primary ROS are short-lived, there is ample evidence that PDT induces prolonged oxidative stress in PDT-treated cells $[25,26]$. The post-PDT oxidative stress stems from (per)oxidized reaction products such as lipids [26] and proteins [27] that have a longer lifetime and, in addition to acutely generated ROS, depletion of intracellular antioxidants [28] and, hence, further exacerbation of already perturbed intracellular redox homeostasis.

The generation of ROS and oxidative stress by PDT leads to the activation of three distinct tumoricidal mechanisms. The first mechanism is based on the direct toxicity of photoproduced ROS, which oxidizes and damages biomolecules and affects organelle and cell function. For example, 8hydroxydeoxyguanosine is a reaction product of ROS with guanosine [29] and may contribute to the induction of DNA damage by PDT [30-38]. Furthermore, 8-oxo-7,8-dihydro-2'guanosine is a product of RNA oxidation reactions that leads to impaired RNA-protein translation [39, 40].

With respect to phospholipids, linoleic acids are prominent targets for ROS-mediated peroxidation [41], yielding 9-, 10-, 12-, and 13-hydroperoxyoctadecadienoic acids as specific products of ${ }^{1} \mathrm{O}_{2}$-mediated linoleic acid oxidation [42]. Other membrane constituents such as cholesterol, $\alpha$-tocopherol, aldehydes, prostanes, and prostaglandins are susceptible to oxidation by type I and type II photochemical reaction-derived ROS [41, 43-46]. The (per)oxidative modifications of phospholipids and membrane-embedded molecules by ROS lead to changes in membrane fluidity, permeability, phasetransition properties, and membrane protein functionality [47-60]. Since many photosensitizers are lipophilic, the oxidation of membrane constituents by PDT is likely a prominent cause of cell death.

In addition to nucleic acids and lipids, most protein residues are also susceptible to oxidation by type I and type II photochemical reaction-derived ROS, which can potentially lead to rupture of the polypeptide backbone as a result of peptide bond hydrolysis, main chain scission, or the formation of protein-protein cross-links [61]. Specific amino acids such as histidine, tryptophan, tyrosine, cysteine, and methionine that may be involved in the active sites of enzymes can be oxidized. Proteins that are most abundantly modified by PDTgenerated ROS include proteins involved in energy metabolism (e.g., $\alpha$-enolase, glyceraldehyde-3-phosphate dehydrogenase), chaperone proteins (e.g., heat shock proteins (HSP)70 and 90), and cytoskeletal proteins (e.g., cytoplasmic actin 1 and filamin A $\alpha$ ) [62]. Besides detrimental effects on protein function, oxidative modification of these biologically essential substrates disrupts the normophysiological redox state of cells, leading to oxidative stress and, in case of excessive damage or stress, cell death via necrosis, apoptosis (reviewed in [63]), or necroptosis [64], depending on which intracellular substrates are most affected by ROS (reviewed in [65]).

Surviving cells may activate adaptation mechanisms in order to (1) restore the intracellular redox homeostasis (antioxidant response), (2) activate a stress response that aids in survival or stimulates apoptosis (immediate early stress response), and (3) facilitate in refolding or degradation of carbonylated proteins (proteotoxic stress response). Autophagy as a result of mitochondrial or ER stress may prevent apoptotic cell death and thereby constitutes a survival mechanism in sublethally damaged tumor cells following PDT [66].

\subsubsection{PDT-induced hypoxia}

The second tumoricidal mechanism of PDT involves the induction of local hypoxia in the irradiated tumor bulk. The acute induction of hypoxia is a result of $\mathrm{O}_{2}$ depletion in consequence to the $\mathrm{O}_{2} \rightarrow{ }^{1} \mathrm{O}_{2}$ or $\mathrm{O}_{2}{ }^{-}$conversion and subsequent oxidation of biomolecules during PDT [67] and the shutdown of tumor vasculature after PDT [68]. The majority of systemic first- and second-generation photosensitizers localize primarily in endothelial cells as well as tumor cells that line the tumor vasculature after short drug-light intervals [69, 70], defined as the time between photosensitizer administration and light delivery. Endothelial photosensitization in particular is associated with vasculature-damaging effects [71-74] that translate to a favorable therapeutic outcome. Prolonged hypoxia due to the destruction of intratumoral vasculature was found to be crucial in the massive induction of cell death following PDT as a result of thrombosis, hemostasis, and cessation of oxygen and nutrient supply (reviewed in [68]). A state of hypoxia or even anoxia reduces the ability of cells to generate ATP by oxidative phosphorylation [75]. As will be reviewed here, hypoxia causes cells to resort to ATP production through anaerobic metabolism to sustain cell function and restore homeostasis and promote angiogenesis to resolve the hypoxic conditions. Cells that are incapable of sustaining ATP production anaerobically due to extensive oxidative stress undergo necrotic cell death (an ATP-independent mode of cell death), which is the strongest trigger for the third tumoricidal mechanism: the antitumor immune response.

\subsubsection{PDT-induced antitumor immune response}

The antitumor immune response, which is triggered by a form of sterile inflammation, constitutes an important process in the post-PDT removal of the treated malignancy. Various studies in mice have shown that activation of the immune system after PDT is necessary for complete eradication of the tumor [76, 77]. The tumor cell death that occurs directly from 
photochemical damage or as a result of vascular shutdownmediated hypoxia/anoxia and hyponutrition is the key precursor event for the antitumor immune response.

The PDT-treated cancer cells die as a result of necrosis, apoptosis [78], necroptosis [64], and/or autophagy [79]. In all modes of cell death, intracellular molecules are released that, following their release, act as so-called damage-associated molecular patterns (DAMPs) [80]. The released molecules also comprise tumor-associated antigens (TAAs) that are otherwise shielded from recognition by immune cells and hence are nonimmunogenic until released [81]. Accordingly, the extracellular DAMPs and TAAs alert cells of the innate and adaptive immune system of impending cellular demise and the presence of malignant tissue, respectively, and consequently trigger a sterile immune response aimed at removing the PDT-treated tumor [82]. A major advantage of the PDTtriggered oncoimmunological pathways is that these pathways can trigger an antitumor immune response mediated by antigen-specific T-cells against distant tumor cells that were not subjected to PDT (referred to as abscopal effects) [83, 84].

\section{Survival pathways activated in tumor cells post-PDT}

The tumor cells that are subjected to sublethal oxidative damage or that are located in tumor regions not affected by vascular shutdown can activate cell survival mechanisms that have been proposed to lie at the basis of therapeutic recalcitrance [17]. We postulate that tumor cell survival following
PDT is attributable to at least five interconnected pathways. These pathways include (1) an antioxidant response mediated by NRF2; (2) a hypoxic survival response mediated by HIF-1; (3) a proinflammatory and angiogenic response mediated by $\mathrm{NF}-\mathrm{KB}$; (4) a proteotoxic stress response mediated by transcription factors HSF1, X-box binding protein 1 (XBP1), activating transcription factor (ATF) 6, and ATF4; and (5) an acute stress response mediated by apoptosis signalregulating kinase 1 (ASK1), its downstream mitogenactivated protein kinase (MAPK) that targets c-Jun N-terminal kinase (JNK) and $\mathrm{p} 38^{\mathrm{MAPK}}$, and the transcription factors of the activator protein 1 (AP-1) family. An overview of the survival pathways is provided in Fig. 2. The following sections will address each of these pathways individually and discuss their potential activation mechanism by PDT, their downstream effects and function, their participation in the PDT response, as well as possible inhibition strategies to reduce their cytoprotective effects and improve the tumoricidal efficacy of PDT.

Some of the survival mechanisms operate by their constitutive activation in cancer cells before PDT, which then prevent cell death following PDT. In other cases, the activation of the survival mechanisms is induced by PDT and may consequently translate to prolonged survival in cells that were subjected to sublethal oxidative damage. Despite the fact that the ROS produced by PDT are generally shortlived (Section 2.1), their secondary metabolites (e.g., (per)oxidized proteins, protein residues, and lipids) can sustainably disrupt cellular redox states in the tumor tissue $[26,28,62]$. This may cause a second wave of cell

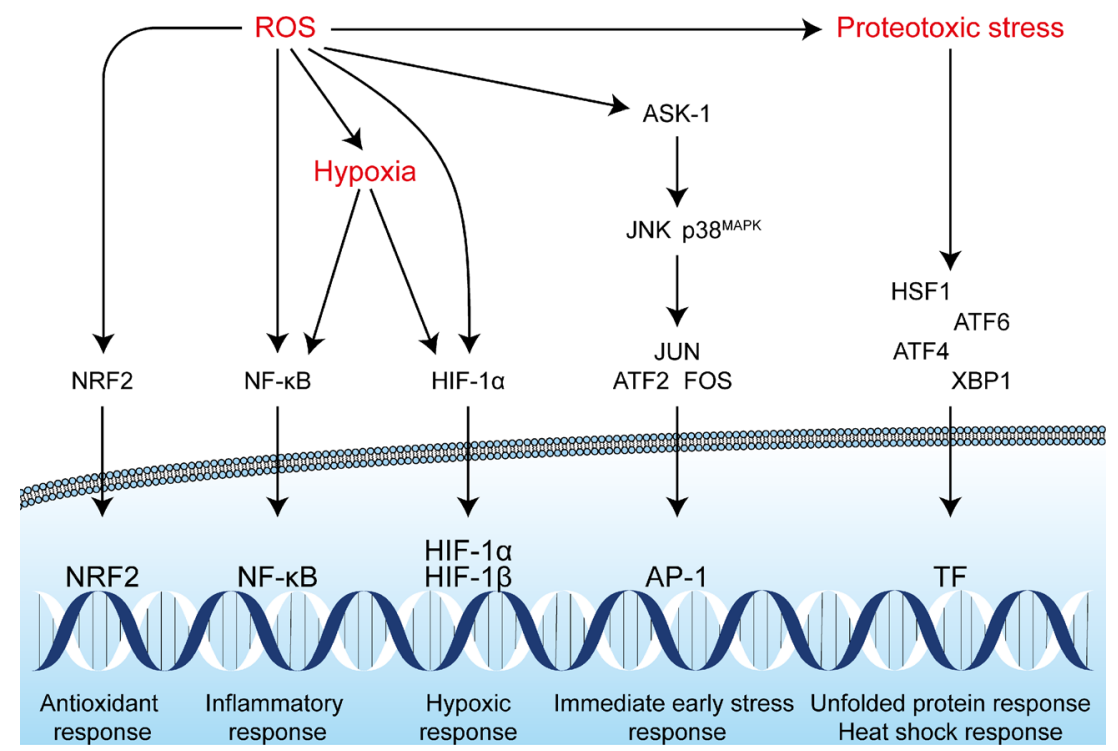

Fig. 2 Reactive oxygen species (ROS)-induced activation of cell survival-related signal transduction pathways in cancer cells following photodynamic therapy (PDT). PDT induces vascular shutdown and oxidation of proteins, which results in hypoxia and proteotoxic stress, respectively. ROS directly trigger the NRF2-mediated antioxidant

response and the ASK1-induced immediate early stress response. Hypoxia and ROS are both involved in the activation of the NF-kB inflammatory response and the HIF-1 hypoxic response. The proteotoxic stress response is characterized by the activation of several transcription factors (TF), including HSF1, ATF4, ATF6, and XBP1 
death, whereby the oxidatively stressed but still viable tumor cells ultimately perish via programmed mechanisms due to the inability to restore cell function and homeostasis [85].

The molecular pathways discussed in this chapter are generally involved in shifting the balance toward cell survival, although in some contexts, these pathways may also stimulate cell death. It should be pointed out that the exact activation mechanisms of the signaling pathways have generally not been studied in the context of PDT, but rather in the context of oxidative stress, ROS, hypoxia, or other pathways. However, since many of these activators have also been implicated in PDT, we propose that these activation mechanisms can also be applied to PDT-treated cells to explain various experimental findings that support a survival-promoting role for these pathways.

\subsection{The NRF2 pathway}

During PDT, ROS are formed that oxidize a plethora of biomolecules and lead to their structural modification and dysfunction. When this occurs on an extensive scale, the oxidative stress culminates in acute cell death. However, when insufficient ROS are produced to induce acute cellular demise, cells will suffer from prolonged oxidative stress whereby the intracellular antioxidative capacity is reduced in the absence of full execution of cell death pathways. Upon exposure to sublethal oxidative stress, cells attempt to restore redox homeostasis through the upregulated production of antioxidants, detoxifying enzymes, as well as phase III drug transporters to mediate the efflux of potentially harmful oxidation products $[86,87]$.

NRF2 is the transcription factor that initiates this antioxidant response, a process that may be important in PDTsurviving tumor cells since it enables the cells to restore intracellular redox homeostasis in a post-PDT microenvironment and enhances the chances for long-term survival. Although NRF2 is a putative repressor of tumorigenesis by protecting cells by detoxifying ROS and ameliorating other stressors that cause malignant transformation [88], the cytoprotective effects of NRF2 are likely to contribute to reduced apoptosisand therapy resistance in tumor cells. Moreover, NRF2 and its downstream gene products are constitutively overexpressed in many tumor types [89], especially in malignant tissues that had been exposed to the carcinogenic effects of oxygen, air pollution, and tobacco smoke [90], thereby predisposing tumor cells to tolerate PDT-induced oxidative stress to a greater extent. In a review on the role of NRF2 in oncogenesis, Gañán-Gómez et al. proposed that NRF2 deregulation in tumor tissue could be attributed to mutations and loss of heterogeneity; hormonal and onocogenic signaling; epigenetic, posttranscriptional, and posttranslational abnormalities; deregulation of autophagy, as well as induction by drugs [90]. Consequently, tumorigenesis is stimulated by aberrant NRF2 signaling that translates to enhanced cell growth, promotion of metastasis, increased survival, and chemoresistance [90]. Accordingly, the following sections discuss the activation mechanism of NRF2 by ROS (Section 3.1.1), the downstream gene targets of NRF2 and their function (Section 3.1.2), the evidence for the participation of the NRF2 pathway in the survival of tumor cells following PDT (Section 3.1.3), as well as potential NRF2 inhibition strategies to reduce tumor cell survival following PDT (Section 3.1.4).

\subsubsection{Activation mechanism of NRF2}

NRF2 is a bZIP transcription factor that is constitutively expressed in most cells and tissue types [91-93]. Under normoxic conditions, NRF2 associates with Kelch-like ECH-associated protein 1 (KEAP1) that is bound to the cytoplasmic cytoskeleton and therefore sequesters NRF2 in the cytosol $[94,95]$. Moreover, KEAP1 binds Cullin-3 that forms a scaffold for E3 ubiquitin ligases to facilitate polyubiquitination and subsequent proteasomal degradation of NRF2. Thus, under normoxic conditions, the antioxidant stress response is inactivated by high levels of cytosolic retention and degradation of NRF2 (reviewed in [86]). During oxidative stress, the NRF2-binding domain of KEAP1 is oxidized at Cys273 and Cys288, resulting in impaired KEAP1 binding to NRF2 [96]. Consequently, free NRF2 accumulates in the cytoplasm where it is activated by oxidation at Cys 183 , after which it is able to translocate to the nucleus [86]. Additional phosphorylation of NRF2 at serine (Ser) 40 by $\mathrm{p} 38 \alpha / \beta$ and/or JNK1, which are also induced by PDT (Section 3.4), may also play a role in the dissociation of the NRF2-KEAP1 complex or the prevention of NRF2-KEAP1 binding [97-99]. Once in the nucleus, NRF2 dimerizes with members of the AP-1 family, such as JUN and musculoaponeurotic fibrosarcoma oncogene homologue (MAF) subfamily proteins $[100$, 101], and binds to antioxidant response element (ARE) sequences to induce the transcription of antioxidant genes. An overview on the activation mechanisms of NRF2 and downstream effects is presented in Fig. 3. An elaborate review on the activation mechanisms of NRF2 is provided in [86].

\subsubsection{Downstream effects of the NRF2 pathway}

The products of NRF2 target genes are involved in the synthesis and redox cycling of antioxidants as well as the removal of potentially harmful oxidation products. The NRF2/AP-1 target genes include $\mathrm{NAD}(\mathrm{P}) \mathrm{H}$ :quinone oxidoreductase 1 (NQO1) and NQO2, heme oxygenase-1 (HO-1, HMOX1), glutamate-cysteine ligase (GCL), microsomal epoxide hydroxylase (EH-1), glutathione S-transferases (GSTs), sulfiredoxin 1 (SRXN1), and carboxylesterase 1A1 (CES1A1) [102]. EH-1 neutralizes epoxides, whereas NQO1 and $\mathrm{NQO} 2$ reduce oxidized quinones to prevent further cell 


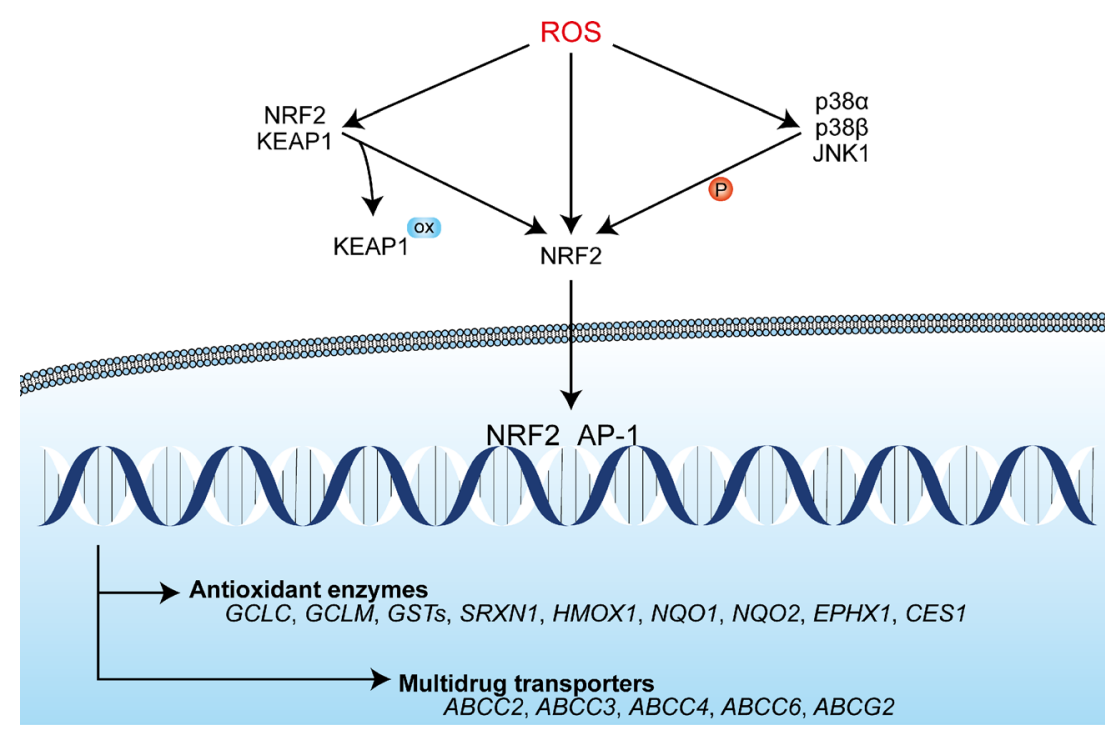

Fig. 3 The activation mechanism of NRF2 and downstream transcription events. Under normophysiological conditions, NRF2 is sequestered in an inactive cytoplasmic complex with KEAP1. Under oxidative stress conditions, ROS mediate the oxidation (ox) of essential cysteines in the NRF2-binding domain of KEAP1, which deters complex formation. NRF2 can be additionally oxidized at Cys 183 by ROS under prooxidative conditions, which enables its nuclear translocation.
Moreover, ROS can activate the ASK1 pathway, in which the MAPKs JNK1 and $\mathrm{p} 38 \alpha / \beta$ phosphorylate (P) NRF2 at Ser40, leading to its activation. Subsequently, NRF2 translocates to the nucleus where it dimerizes with AP-1 transcription factors (Section 3.4.2) and initiates the transcription of antioxidant enzymes (e.g., glutathione synthesis) and multidrug transporters $(A B C C 2, A B C C 3, A B C C 4, A B C C 6$ and $A B C G 2)$ damage by these reactive species [103, 104]. CES1A1 hydrolyzes esters and thioesters [105]. HO-1 neutralizes certain types of ROS directly as well as oxidized metabolites (lipid radicals) indirectly by producing the antioxidant molecule bilirubin from heme [106, 107]. Moreover, proteins involved in the reduction and reactivation of radical scavengers such as glutathione (GSH) and peroxiredoxins are upregulated by NRF2, including GCL (subunits GCLC and GCLM), GSTs, and SXRN1 [108, 109]. NRF2 further upregulates ATPbinding cassette subfamily $\mathrm{C}$ (ABCC) 2, 3, 4, and 6 (also known as multidrug resistance proteins (MRPs)) and the multidrug efflux pump ATP-binding cassette subfamily G member 2 (ABCG2), which mediate efflux of organic anions and glutathionylated, glucuronidated, and/or sulfated (bio)molecules (reviewed in [102, 110-112]). Such biomolecules are the products of oxidative stress that are potentially harmful by themselves [102]. MRPs may therefore be crucial for the detoxification of tumor cells that have survived the initial PDT-induced ROS attack and can aid in restoring the intracellular redox balance.

HO-1 in particular has been linked to cancer cell survival following PDT. Besides being upregulated by NRF2, HO-1 (encoded by the HMOXI gene) is upregulated by HIF-1 [113], which is also induced by PDT (Section 3.3). The function of HO-1 is to convert mitochondrially produced heme into carbon monoxide (CO) and biliverdin, of which the latter is reduced by biliverdin reductases to bilirubin [114]. Bilirubin scavenges peroxidized lipids $[115,116]$ and may significantly contribute to tumor cell survival following PDT by terminating lipid oxidation chain reactions. Furthermore, at low concentrations, $\mathrm{CO}$ possesses vasodilating, proangiogenic, anti-inflammatory, and antiapopotic properties, which can contribute to angiogenesis, tumor survival, and tumor regeneration in vivo $[117,118]$. Although the degradation of heme to bilirubin also liberates $\mathrm{Fe}^{2+}$ that contributes to a prooxidant state, the release of $\mathrm{Fe}^{2+}$ by $\mathrm{HO}-1$ was found to concomitantly increase the transcription of ferritin [119], which chelates and neutralizes free $\mathrm{Fe}^{2+}[120]$.

Another major pathway augmented by NRF2 is the GSH synthesis pathway, which yields an effective redox machinery aimed at scavenging ROS and neutralizing reactive intermediates such as oxidized protein residues (by glutathionylation) [121]. Synthesis of the GSH tripeptide occurs by ligation of Lglutamate and L-cysteine by GCL and addition of glycine by GSH synthetase. GSH can reduce ROS through oxidation of its thiol moiety ( $\mathrm{GSH} \rightarrow \mathrm{GS} \bullet$ ), after which the reactive thiol is neutralized by GS-GS homodimerization (GSSG) with another GS• through disulfide bridge formation. Recycling of GSSG to GSH is catalyzed by GSSG reductase (reviewed in [121]). GSH can also react with oxidized cysteine residues, resulting in protein glutathionylation and subsequent cellular efflux via proteins of the MRP family [110]. Moreover, GSTs of different classes are upregulated by NRF2, which are responsible for the glutathionylation of oxidized proteins resulting in increased MRP transporter-mediated efflux of glutathionylated peptides [122]. Another role for GSTs is to inhibit molecular constituents in the ASK1 pathway, including ASK1 (by GSTM), JNK (by GSTP/GSTA), and tumor 
necrosis factor receptor associated factor 2 (TRAF2) (by GSTP), although the inhibitory efficacy decreases upon oxidative stress [122]. This may prevent prolonged activation of the ASK1 pathway and could stimulate cell survival as is discussed in Section 3.4. In sum, the activation of NRF2 is essential for the production of proteins involved in GSH synthesis and redox regulation, as well as the neutralization of oxidative compounds and their cellular efflux.

\subsubsection{Role of the NRF2 pathway in PDT}

Although NRF2 activation by ROS is well-established, its activation by PDT has been sparsely investigated. Nuclear translocation and thus activation of NRF2 was observed by Kocanova et al. in human bladder cancer (T24) cells and human cervical cancer (HeLa) cells following hypericin-PDT [85]. Furthermore, NRF2 target genes were overexpressed in various cancer cells after PDT, which include HO-1 [123], GCLC and GCLM subunits of GCL [124], NQO1 [124, 125], and ABCG2 [111]. The inhibition of $\mathrm{p} 38^{\mathrm{MAPK}}$ (p38 $\alpha$ and $\mathrm{p} 38 \beta$, Section 3.4.2) with PD169316 reduced HO-1 messenger RNA (mRNA) levels and increased the susceptibility of T24 cells to PDT [85]. These findings indicate that NRF2 is activated following PDT, that $\mathrm{p} 38^{\mathrm{MAPK}}$-mediated phosphorylation enhances the activity of NRF2 post-PDT, and that the expression of HO-1 by NRF2 is cytoprotective. Several reports have corroborated HO-1-mediated cytoprotection following PDT [123, 126, 127]. However, HO-1 was also found to be induced by aminolevulinic acid (ALA) prior to PDT [111], and targeted knockdown of HO-1 has been related to reduced intracellular protoporphyrin IX (PPIX) accumulation [128], indicating that HO-1 can both inhibit PS accumulation as well as reduce the PDT response. Interestingly, of the MDR proteins induced by NRF2, at least ABCG2 has been confirmed to facilitate cytoprotection against PDT by mediating the cellular efflux of photosensitizers PPIX, pyropheophorbide A, and benzoporphyrin derivative monoacid ring A [129] but not mesotetrahydroxyphenylchloride and porfimer sodium [130].

\subsubsection{Inhibition strategies for NRF2 and its downstream targets}

Retinoic acid has been identified as an inhibitor of NRF2 in human mammary carcinoma (MCF-7) cells transfected with an ARE-luciferase reporter construct. Retinoic acid abolished the expression of genes with ARE sequences in their promoter regions [131] but did not affect the nuclear translocation or degradation of NRF2. Rather, retinoic acid inhibited the function of NRF 2 by activating retinoic acid receptor $\alpha(\mathrm{RAR} \alpha)$ in the nucleus. RAR $\alpha$ sequesters NRF2 in the nucleus, thereby inhibiting the association between NRF2 and ARE sequences
[131] (Table 1). Unfortunately, not much is known about the binding specificity of retinoic acid, nor has retinoic acid or any of its analogs been studied in the context of PDT. Nevertheless, retinoic acid and its analogs are also involved in the inhibition of AP-1 transcription factors (Section 3.4.2.2 Prolonged downstream effects of ASK1 activation), which constitute the main dimerization partners for NRF2. Thus, PDT with RAR $\alpha$ activators may potentially enhance the cytotoxic effects of PDT by inhibiting both AP-1 and NRF2 survival signaling.

In addition to inhibiting NRF2-mediated gene expression, the downstream gene products of NRF2, such as HO-1 and members of the GSH antioxidant machinery, may also be successfully inhibited by small molecular compounds (e.g., Zn-protoporphyrin IX (ZnPP) [132, 165], Table 1). Inasmuch as HO- 1 catalyzes the degradation of heme into the antioxidants bilirubin and $\mathrm{CO}$, inhibition of HO-1 with $\mathrm{ZnPP}$ during PDT is expected to enhance tumoricidal efficacy. Indeed, HO-1 inhibition with $2.5 \mu \mathrm{M}$ ZnPP considerably reduced cell viability following porfimer sodium-PDT in both human (MDAH2774) and murine (C26) colon carcinoma cell lines. The addition of bilirubin or $\mathrm{CO}$ could not rescue cells from PDT-induced cell death upon HO-1 inhibition, suggesting a more elaborate role of HO-1 in the survival of tumor cells than merely the synthesis of antioxidants [123]. Similar results with HO-1 inhibition were obtained in WM541Lu human melanoma cells subjected to ALA-PDT, where the addition of anti-HO-1 siRNA (24 h prior to PDT) or tin-PPIX (SnPP [133]) increased the susceptibility of these cells to PDT [126]. SiRNA-mediated knockdown of HO-1 also increased the susceptibility of UM-UC-3 (but not T24, KU7, UM-CU-2, and UM-CU-4) human urothelial carcinoma cell lines to ALA-PDT [128]. With respect to other NRF2-upregulated antioxidants, Kimani et al. tested several inhibitors of the glutathione redox system and ROS scavenging enzymes (superoxide dismutases (SODs) and catalase) to increase the efficacy of disulfonated aluminum-phthalocyanine-PDT of MCF-7 breast cancer cells [166]. Diethyl-dithiocarbamate (DDC) and 2-methoxyestradiol (2-ME) were used as inhibitors of $\mathrm{Cu}-\mathrm{SOD} / \mathrm{Zn}-\mathrm{SOD}$ [167] and Mn-SOD (although 2-ME has been shown not to inhibit Mn-SOD [134]), respectively (Table 1). L-Buthionine sulfoximine (BSO) was used as an inhibitor of GCL (glutathione synthesis [135]) and 3-amino1,2,4-triazole (3-AT) as an inhibitor of catalase [136] (Table 1). All inhibitors were efficient in exacerbating PDT-induced apoptosis. The strongest effects were observed when a combination of BSO with either 3-AT or 2-ME was used, which achieved similar results as when all the inhibitors were combined. This suggests that the inhibition of $\mathrm{H}_{2} \mathrm{O}_{2}$ scavenging (by inhibition of catalase) and $\bullet \mathrm{OH}$ scavenging (by inhibition of GCL and glutathione synthesis) and inhibition of the enzymatic dismutation of $\mathrm{O}_{2}{ }^{-}$to $\mathrm{H}_{2} \mathrm{O}_{2}$ (by SODs) significantly increase the susceptibility of cells to 
Table 1 Overview of pharmacological agents that are used for the inhibition of select targets in PDT-induced cytoprotective pathways

\begin{tabular}{|c|c|c|c|c|}
\hline Pathways & Target & Inhibitor & Mechanism & Reference \\
\hline NRF2 & NRF2 & Retinoic acid (and analogs) & Inhibition of DNA- binding due to sequestration by RAR $\alpha$ & [131] \\
\hline NRF2 & $\mathrm{HO}-1$ & $\mathrm{ZnPP}$ & Nonreversible antagonist & [132] \\
\hline NRF2 & HO-1 & SnPPIX & Nonreversible antagonist & [133] \\
\hline NRF2 & $\mathrm{Cu}-\mathrm{SOD} / \mathrm{Zn}-\mathrm{SOD}$ & $\mathrm{DDC}$ & Chelation of $\mathrm{Cu}(\mathrm{II})$ and $\mathrm{Zn}(\mathrm{II})$ & [167] \\
\hline NRF2 & Mn-SOD & 2-ME & Does not inhibit Mn-SOD but increases superoxide levels & {$[134]$} \\
\hline NRF2 & GCL & BSO & Nonreversible antagonist & {$[135]$} \\
\hline NRF2 & Catalase & 3-AT & Nonreversible antagonist & {$[136]$} \\
\hline NF-kB & RELA (NF-kB) & Parthenolide & Alkylation at Cys 38 , inhibition of DNA binding & {$[137]$} \\
\hline $\mathrm{NF}-\mathrm{kB}$ & NF- $\mathrm{kB}$ & Panepoxydone & Inhibition of I $\mathrm{kB}$ phosphorylation & [138] \\
\hline $\mathrm{NF}-\mathrm{kB}$ & NF- $\kappa \mathrm{B}$ & Bay 11-7082 & Inhibition upstream of IKK & [139] \\
\hline$N F-\kappa B$ & NF- $к B$ & DHMEQ & Inhibition of DNA binding and nuclear localization & {$[140]$} \\
\hline NF-kB & $\mathrm{NF}-\mathrm{kB}$ & $\alpha$-Ketoglutarate & Reactivation of PHD proteins & {$[141]$} \\
\hline NF-kB & $\mathrm{COX}-2$ & NSAIDs (e.g., ibuprofen, celecoxib) & Reversible antagonist & [142] \\
\hline NF-kB & STAT3 & STA-21 & Inhibition of DNA binding & [143] \\
\hline$N F-\kappa B$ & STAT3 & WP1066 & Dephosphorylates STAT3 and causes nuclear export & [144] \\
\hline NF- $k \mathrm{~B}$ & Survivin & LY218130B & RNAi & [145] \\
\hline NF-kB & Survivin & YM155 & Inhibition of transcription & {$[145]$} \\
\hline NF-kB & Survivin & Terameprocol (EM1421) & Inhibition of transcription & {$[145]$} \\
\hline NF- $k \mathrm{~B}$ & IL-6/sIL-6R & sgp130Fc & Sequestration of sIL-6R & [146] \\
\hline NF-kB & MMP (broad spectrum) & Prinomastat & Chelation of $\mathrm{Zn}(\mathrm{II})$ in the catalytic domain & {$[147]$} \\
\hline HIF-1 & HIF-1 & Amphotericin B & Increased activity of FIH & [148] \\
\hline HIF-1 & HIF-1 & Echinomycin & Inhibition of DNA binding & [149] \\
\hline HIF-1 & HIF-1 & $\alpha$-Ketoglutarate & Reactivation of PHD proteins & {$[141]$} \\
\hline HIF-1 & HIF-1 & Curcumin & Oxidation and proteasomal degradation of HIF- $1 \beta$ & {$[150]$} \\
\hline HIF-1 & HIF-1 & Acriflavine & Binding to HIF- $1 \alpha$ dimerization domain & {$[151]$} \\
\hline ASK1 & AP-1 & Retinoic acid (and analogs) & Inhibition of DNA binding (does not involve RAR $\alpha$ ) & {$[152]$} \\
\hline ASK1 & JNK1 & SP600125 & Reversible ATP antagonist & {$[153]$} \\
\hline ASK1 & $\mathrm{p} 38 \alpha / \beta$ & SB202190 & Reversible ATP antagonist & {$[154]$} \\
\hline ASK1 & $\mathrm{p} 38 \alpha / \beta$ & $\mathrm{Sb} 203580$ & Reversible ATP antagonist & [154] \\
\hline ASK1 & $\mathrm{p} 38 \alpha / \beta$ & PD169316 & Reversible ATP antagonist & {$[154]$} \\
\hline UPR & HSP90 & Geldanamycin (17-AAG) & ATP antagonist & {$[155]$} \\
\hline UPR & HSP90 & CNF2024/BIIB021 & ATP antagonist & {$[156]$} \\
\hline UPR & HSP90 & NVP-AUY922 & Complex formation with HSP70 & [157] \\
\hline UPR & HSP90 & SNX-5422 & ATP antagonist & {$[158]$} \\
\hline UPR & HSP90 & STA-9090 & ATP antagonist & [159] \\
\hline UPR & HSP70 & SubA & Antagonist & {$[160]$} \\
\hline UPR & HSP70 & VRS-155008 & ATP antagonist & {$[161]$} \\
\hline UPR & HSF1 & KRIBB11 & Inhibition of transcriptional activity & {$[127]$} \\
\hline UPR & Proteasome & Bortezomib & Antagonist of catalytic site & [162] \\
\hline UPR & IRE1/ATF6 & 4-Phenylbutyric acid (and analogs) & Unknown & {$[163]$} \\
\hline UPR & PERK & GSK-2656157 & APP antagonist & [164] \\
\hline
\end{tabular}

PDT [166]. In conclusion, the preemptive inhibition of both the bilirubin and glutathione synthesis pathways revealed a protective effect of these pathways on the survival of tumor cells following PDT, altogether indicating that the NRF2 pathway counteracts the cytotoxicity of PDT.

\subsubsection{Concluding remarks}

NRF2 is the main trigger for the antioxidant stress response that restores the intracellular redox status toward normophysiological levels in PDT-surviving cells. The 
antioxidant stress response is activated by oxidative stress (Section 3.1.1) and culminates in the neutralization, modification, and cellular export of oxidized/oxidizing compounds and/or potentially hazardous products of oxidation reactions (Section 3.1.2). Given the experimental evidence that NRF2 is activated following PDT (Section 3.1.3) and that inhibition of NRF2-upregulated processes potentiates the efficacy of PDT (Section 3.1.4), NRF2 seems to be an important mediator of tumor cell survival following PDT.

It is important to realize that the short-lived ROS produced during PDT cannot be scavenged by antioxidants produced downstream of the NRF2 signaling pathway since these are produced long past the half-lives of these ROS, unless there is constitutive overexpression of this pathway. Rather, NRF2 may act as an essential factor for PDT-surviving tumor cells to restore the redox imbalance and promote prolonged survival in a post-PDT microenvironment. Moreover, since NRF2upregulated proteins HO-1, MDR1, and ABCG2 are often upregulated in many cancer types, NRF2 is likely constitutively active in tumor cells, potentially desensitizing these cells to PDT and thereby playing an instrumental role in also neutralizing the first wave of ROS directly produced by PDT. Therefore, NRF2 inhibition strategies aimed at preventing NRF2 activity prior and/or post-PDT may prove to be beneficial for the enhancement of PDT efficacy as a result of impaired tumor cell adaptation to oxidative stress.

\subsection{The NF-KB pathway}

The NF- $\mathrm{KB}$ transcription factor family is mainly involved in the communication between tissue cells and the immune system. Both intracellular and extracellular signals are translated by NF-KB into transcriptomic responses that ultimately enable tumor cells to attract and support immune cells. NF- $\mathrm{kB}$ plays a role in apoptosis, inflammation, proliferation, and activation of the HIF-1 response [168]. Therefore, the activation of this pathway after PDT supports the survival of tumor cells by preventing apoptosis and promoting angiogenesis [169]. However, PDT may also repress NF-kB activity through redox modifications under severe oxidative stress as well as tumor necrosis factor $\alpha$ (TNF- $\alpha)$ signaling, which is one of the main transcriptional targets of NF- $\mathrm{KB}$, that is concurrently triggered after PDT [170]. As such, NF- $\mathrm{KB}$ represents a complicated survival pathway that may be both activated and repressed by PDT, depending on the severity of the oxidative insult and the interaction with additional signaling pathways.

The following sections will discuss the potential activation and repression mechanisms of NF-KB (Section 3.2.1), its downstream transcriptional effects after activation, and the function of several of the upregulated proteins (Section 3.2.2). After a brief summary of the available evidence for the participation of NF-KB in the response of tumor cells to PDT
(Section 3.2.3), an outline of possible inhibition strategies for NF- $\mathrm{kB}$ and its downstream gene products is provided (Section 3.2.4).

\subsubsection{Activation mechanisms of $N F-\kappa B$}

NF-KB comprises a family of proteins that include reticuloendotheliosis (REL) A, RELB, and c-Rel, as well as NF-kB1 and NF-kB2 [171, 172]. Two types of heterodimeric complexes can be formed from these proteins, each induced by different stimuli. NF-KB transcription factors composed of RELA, c-REL, and NF-kB1 are activated in the presence of proinflammatory cytokines and/or hypoxia. NF- $\mathrm{KB}$ complexes composed of RELB and NF- $\mathrm{KB} 2$ are induced solely by TNF- $\alpha$. Both complexes mediate the transcription of similar target genes that contain $\mathrm{KB}$ elements in their promoter region and thus initiate an inflammatory response to, e.g., ROS and TNF- $\alpha$ [172]. Under normal conditions, NF- $\mathrm{KB}$ transcription factors are retained in the cytosol by inhibitors of $\mathrm{KB}$ (IKB) [168]. NF- $K B$ is activated when I $K B$ is phosphorylated by the IKB kinase (IKK) complex at Ser32 and Ser36, which results in the ubiquitination and degradation of IKB and corollary release and nuclear translocation of NF-KB [172]. Accordingly, the IKK complex plays a major role in the activation of NF-kB. The IKK complex is able to deactivate the IKB protein in response to three independent factors, namely in response to ROS, hypoxia, and TNF- $\alpha$ (Fig. 4).

NF- $K B$ activation by ROS ROS is a primary activator of NF-KB via oxidation of the IKK complex. IKK is composed of two subunits with kinase activity, termed IKK $\alpha$ and IKK $\beta$, which are held together by one or two regulatory subunits called IKK $\gamma$ (or NF-kB essential modulator, NEMO) [172]. The exact mechanism underlying IKK activation by ROS is relatively unclear and appears to be cell type-specific. In CME and Jurkat T-lymphocytes, $\mathrm{H}_{2} \mathrm{O}_{2}$ treatment induced phosphorylation of IKB via IKK, of which the activity was dependent on SH2-containing inositol 5'-phosphatase 1 (SHIP-1) [173]. However, in various human cancer cell lines and ROSinducing treatments, the ROS-dependent phosphorylation of IKK involves protein kinase D (PKD), sarcoma (SRC), and Abelson murine leukemia viral oncogene (ABL) [174, 175], c-SRC [176], MAPK kinase (MKK)3 [177] (downstream of ASK1, Section 3.2.4), or NF-kB inducing kinase (NIK) [178]. Additionally, IKK $\gamma$ contains two redox-sensitive cysteines (Cys54 and Cys347) that may be instrumental in the formation of IKK $\gamma$ dimers in the presence of ROS, leading to enhanced complex formation and IKK $\alpha / \beta$ phosphorylation [179] (reviewed in [180]). 


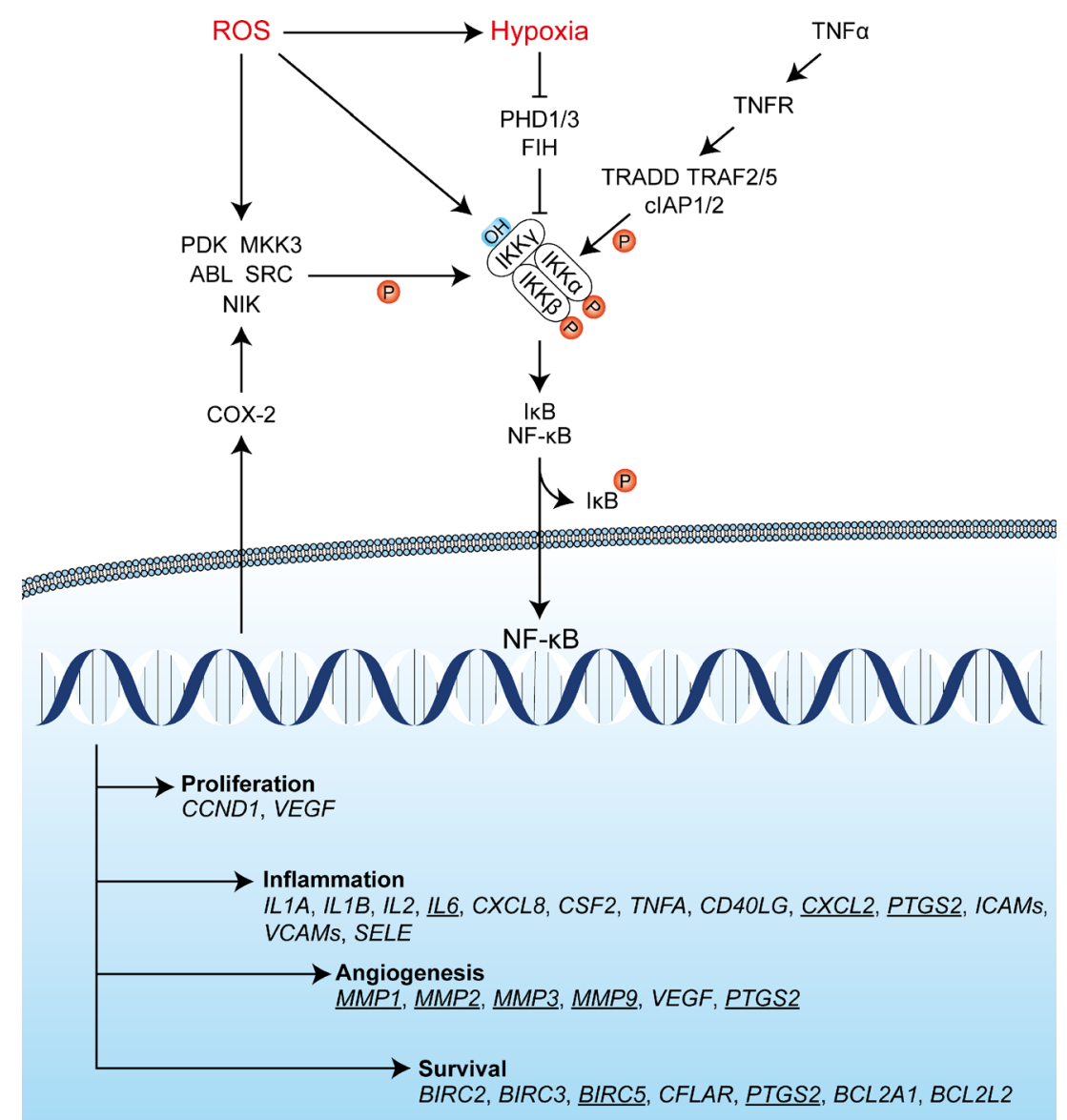

Fig. 4 Potential activation mechanisms of NF-kB in response to PDT. ROS may activate IKK directly by oxidizing redox-sensitive cysteines on $\mathrm{IKK} \gamma$. Alternatively, IKK $\alpha / \beta$ may be phosphorylated by kinases such as $\mathrm{PKD}, \mathrm{ABL}, \mathrm{SRC}$, NIK, and/or MKK3 in response to oxidative stress. Hypoxia is likely a coactivator of the IKK complex, since depletion of oxygen $\left(\mathrm{O}_{2} \rightarrow\right.$ ROS $)$ renders PHD1/3 and FIH dysfunctional, as a result of which hydroxylation of IKK $\gamma$ cannot occur and IKK $\gamma$ is no longer targeted for proteasomal degradation by VHL-mediated polyubiquitination. Finally, in the presence of TNF- $\alpha$, the TNFR becomes activated and triggers the assembly of a complex in which TRADD, TRAF2 $/ 5$, and cIAP1/2 promote the phosphorylation of $\mathrm{IKK} \alpha / \beta$. A stabilized and activated IKK complex phosphorylates IKB,

NF- $\kappa$ B activation by hypoxia A second mechanism that may contribute to NF- $\mathrm{KB}$ activation following PDT is hypoxia. The hypoxic response leading to increased NF- $\mathrm{kB}$ activity is quite similar to the activation of HIF-1 in that the inhibition of the hydroxyl transferase activity of prolyl hydroxylase domain proteins (PHD) 1 and PHD3 and possibly, but less prominently, factor inhibiting HIF-1 (FIH) play a role in the activation of IKK (reviewed in [181]). Although unequivocal experimental evidence is currently lacking, NF- $\mathrm{kB}$ activation by hypoxia is most likely facilitated by the hydroxylation of IKK. Similar hydroxylation-sensitive sites that were found on HIF- $1 \alpha$ have been identified on IKK $\alpha$ and IKK $\beta$ [182], which interact with Von Hippel-Lindau (VHL) protein and subsequently promote polyubiquitination and proteasomal degradation [183]. Thus, it appears that PHD1 and PHD3 which dissociates from the NF- $\mathrm{kB}$ complex and relieves its sequestration in the cytoplasm. Upon release, NF-KB translocates to the nucleus to induce a transcriptional response that promotes proliferation, inflammation, angiogenesis, and survival. Via COX-2, EGFR signaling activates of a variety of kinases (e.g., PKD, MKK3, ABL, SRC, NIK) that in turn phosphorylate and activate the IKK complex. Active NF- $\mathrm{BB}$ transcription factors induce the transcription of genes involved in proliferation, inflammation, angiogenesis, and survival. The underlined genes have been extensively investigated in relation to PDT and are discussed in detail in the main text. CCND1 encodes cyclin D1, PTGS2 encodes $\mathrm{COX}-2$, and BIRC5 encodes survivin

are able to hydroxylate and inactivate IKK complexes during normoxia and that this inactivation capacity is lost during hypoxia, potentially resulting in reduced proteasomal degradation of IKK. It should be noted that reduced degradation of the IKK complex may not be sufficient to confer NF-KB activation and that it has been proposed that hypoxia does not play a major role in the activation of NF-kB [168]. However, hypoxia may act in a costimulatory manner for the ROS(Section 3.2.1.1 NF-kB activation by ROS) or TNF- $\alpha$ mediated activation of NF-KB [181] (Section 3.2.1.3 NF-KB activation by TNF- $\alpha$ ).

NF- $\kappa$ B activation by TNF- $\alpha$ Thirdly, NF- $\kappa B$ can be induced via paracrine/autocrine signaling by TNF- $\alpha$ from tumor cells and chemoattracted immune cells. TNF- $\alpha$ is a downstream gene 
target of both AP-1 and NF- $\mathrm{KB}$ and thus provides a positive feedback loop for NF- $\mathrm{KB}$ activation as well as activation of the ASK1 pathway (Section 3.4). TNF- $\alpha$ binds the TNF receptor (TNFR) that subsequently forms activated homodimers. A signaling complex is formed by the recruitment of TNFR associated with death domain (TRADD), TRAF2/5, and cytosolic inhibitor of apoptosis (cIAP) $1 / 2$. The complex associates with a linear ubiquitin assembly complex that polyubiquitinates RIP1 and IKK $\gamma$, forming an active IKK complex. The active IKK complex is further activated by ubiquitinated RIP1, which in turn leads to the activation of TGF- $\beta$-activated kinase 1 (TAK1) and consequent phosphorylation of IKK $\alpha$ and IKK $\beta$. The activated IKK complex also phosphorylates and inactivates I $\mathrm{B}$, triggering the release and nuclear translocation of the NF- $\mathrm{KB}$ transcription factor complex (reviewed in [184]).

\section{NF- $k B$ inhibition by ROS and TNF- $\alpha$ during severe oxi-} dative stress In contrast to the activatory capacity of ROS and TNF- $\alpha$ described above, severe forms of oxidative stress and/ or the combination of oxidative stress and TNF- $\alpha$ signaling inhibit the activity of NF-kB and promote cell death. Whereas minor or moderate levels of oxidative stress lead to NF- $\mathrm{kB}$ activation (Section 3.2.1.1 NF- $\mathrm{kB}$ activation by ROS), severe oxidative stress has a detrimental effect on NF- $\mathrm{KB}$ activity [185]. Critical cysteines in NF-KB complexes, such as Cys62 on RELA, are susceptible to oxidation and subsequent glutathionylation or nitrosylation, which impairs DNA binding and transcriptional activity [186, 187]. Additionally, IKK $\alpha$ and IKK $\beta$ contain redox-sensitive Cys179, which can be oxidized by $\mathrm{H}_{2} \mathrm{O}_{2}$ and reduce IKK kinase activity [188]. These findings suggest that antioxidants produced de novo via e.g., the NRF2 pathway may facilitate NF-KB activation following a severe prooxidative insult such as PDT by ameliorating the oxidative stress, although more research is required to corroborate this claim. TNF- $\alpha$ exerts its anti-NF-KB effects primarily via mitochondrial ROS production, which may elevate the extent of preexisting moderate oxidative stress to severe oxidative stress and consequent NF- $\mathrm{KB}$ inhibition via the abovementioned processes. For example, TNF- $\alpha$ treatment was shown to cause oxidative stress, the cytotoxicity of which could be repressed by the addition of antioxidants [189]. Inhibition of NF-KB by TNF- $\alpha$-induced oxidative stress stimulates cell death via prolonged activation of JNK1, given that NF-KB target gene products such as A20 and growth arrested and DNA damage (GADD) $45 \beta$ typically inhibit JNK1 activity. As such, ROS have been considered to act as a secondary messenger in TNF- $\alpha$-induced cell death (reviewed in [185]).

The ROS-dependent activation of the NF-KB pathway has several important biological and clinical implications for PDT. Laser irradiation of tissue is characterized by light intensity attenuation with increasing depth as a result of light scattering and absorption [190], resulting in fluence gradients during
PDT. Inasmuch as the extent of ROS production is proportional to the fluence [78], the cancer cells in the more distally located regions of the tumor may exhibit less ROS generation during PDT and hence are subject to a lower degree of oxidative stress than the tumor cells most proximal to the light source. Accordingly, irradiation of bulky tumors may yield a fraction of cancer cells that undergoes cell death without the activation of ROStriggered survival pathways, whereas another fraction of cancer cells, located mainly at the deep periphery of the target tissue, may suffer from oxidative stress but survive as a result of ROSmediated activation of e.g., NF-kB-mediated survival pathways. The latter fraction of cancer cells is particularly important therapeutically inasmuch as these cells may cause tumor regrowth and metastasis after PDT.

\subsubsection{Downstream effects of the $N F-\kappa B$ pathway}

The different NF- $\mathrm{KB}$ transcription factor complexes essentially share the same target genes that are associated with cell proliferation, inflammation, angiogenesis, and survival [172] (Fig. 4). NF-kB transcription factors induce cell proliferation (upregulation of cyclin D1 (encoded by $C C N D 1$ ) and VEGF); cause inflammatory cells to be recruited toward the tumor site (via the production and secretion of interleukin (IL)- $1 \alpha / \beta$, IL2, IL-6, IL-8 (CXCL8), granulocyte-macrophage colony stimulating factor (GM-CSF, CSF2), TNF- $\alpha$, cluster of differentiation 40 ligand $(C D 4 O L G)$, chemokine $\mathrm{C}-\mathrm{X}-\mathrm{C}$ motif ligand (CXCL) 2, and cyclooxygenase 2 (COX-2, encoded by prostaglandin synthase 2 (PTGS2)); trigger angiogenesis by upregulation of matrix metalloproteinases (MMPS), VEGF, and PTGS2; and facilitate inflammatory cell binding via selectin E (SELE), intercellular adhesion molecule (ICAMS), and vascular cell adhesion molecule (VCAMs) [168, 172, 191-193]. The role of NF-KB target gene products ICAM and VCAM appears to be controversial insofar as PDT reduced gene and protein expression levels despite activation of NF-KB [194, 195]. Of the inflammation-associated proteins, IL-6 plays an important role in tumor cell survival following PDT, as discussed in Section 3.2.2.4 IL-6, whereas TNF- $\alpha$ is also directly responsible for inducing cell death via apoptosis and necrosis pathways, as discussed in Section 3.2.2.3 TNF- $\alpha$. To ensure survival of immune cells in a hypoxic environment, NF-KB desensitizes cells to apoptosis through the upregulation of cIAP1 (baculoviral inhibitor of apoptosis repeatcontaining 2, BIRC2), cIAP2 (BIRC3), and survivin (BIRC5) as well as CFLAR, COX-2, and antiapoptotic members of the BCL2 family (BCL2A1, BCL2L1) [192, 196]. Especially survivin and COX-2 have been implicated in cell survival following PDT (Sections 3.2.2.1 COX-2 and 3.2.2.2 Survivin). In addition to these antiapoptotic proteins, NF-kB triggers HIFlA transcription that promotes immune and tumor cell survival in a hypoxic environment as a result of the upregulated production of HIF- 
1 transcription factor [197] (Section 3.3). NF-kB further initiates a negative feedback loop toward its own activity by inducing the expression of IKB subunits and the NF-KB inhibitor A20 [172, 198].

Overall, NF- $\mathrm{KB}$ stimulates tumor cell survival by inhibiting apoptosis and recruiting the immune system to facilitate angiogenesis and promote cell proliferation. The induction of $\mathrm{NF}-\mathrm{KB}$ and the consequent production of cytokines may also be essential to the antitumor immune response (Section 2.2.3), which is essential for complete tumor eradication $[76,77]$ and long-term deterrence of tumor regrowth [199].

COX-2 COX-2 (encoded by PTGS2) is overexpressed in many types of cancer and is generally associated with reduced patient survival [200]. The promoter sequence of COX-2 contains binding sites for NF- $\mathrm{kB}$, HIF-1, ATF2, FBJ murine osteosarcoma viral oncogene homologue (FOS), and JUN [201-203], making it a downstream target of three major survival pathways that are induced by PDT. The main function of COX-2 is to convert arachidonic acid to prostaglandin $\mathrm{H}_{2}$ $\left(\mathrm{PGH}_{2}\right)$, which is further metabolized into $\mathrm{PGE}_{2}, \mathrm{PGF}_{2 \alpha}$, $\mathrm{PGI}_{2}$, and thromboxane $\mathrm{A}_{2}\left(\mathrm{TBA}_{2}\right)$ [204]. $\mathrm{PGE}_{2}$ induces growth of tumor epithelial cells by binding the $\mathrm{PGE}_{2}$ receptor and activating rat sarcoma protein (RAS) and phosphatidyl inositol 3 kinase (PI3K), which activate signaling pathways that ultimately lead to proliferation and cell division [205-207]. In addition, prostaglandins induce SRC, epidermal growth factor receptor (EGFR), MMP2, and C-C chemokine receptor 7 (CCR7) to stimulate cell migration [208-210]. Prostaglandins also stimulate angiogenesis by facilitating the production of VEGF, fibroblast growth factor (FGF)2, and molecules involved in immune cell chemotaxis and adhesion, including chemokine $\mathrm{C}-\mathrm{X}-\mathrm{C}$ motif ligand (CXCL)1, integrin $\alpha \mathrm{V} \beta 3$, chemokine $\mathrm{C}-\mathrm{C}$ motif ligand (CCL)2, and CXC receptor 4 [207, 211-213].

Survivin Survivin is a member of the inhibitor of apoptosis protein (IAP) family, which also comprises NLR family apoptosis-inhibitory protein, cIAP1, cIAP2, X-linked IAP (XIAP), and livin [214]. The expression of the genes that encode these proteins (BIRC1-4 and BIRC7) is generally induced by transcription factor 4 , signal transducer and activator of transcription 3 (STAT3), as well as the PDT-induced transcription factors NF-KB and HIF-1 (reviewed in [215]). Survivin is considered a nodule protein; a protein that stands at the center of many signaling pathways and plays a role in many cellular processes. In general, survivin stimulates cell division in the mitotic phase of the cell cycle and suppresses apoptosis (reviewed in [145]). Survivin also partakes in a chromosomal passenger complex that binds kinetochores and stimulates spindle formation to facilitate chromosome segregation during mitosis. The antiapoptotic role of survivin is reflected by its inhibition of caspase 9 [216] and prevention of XIAP degradation [145, 217]. Furthermore, alternatively spliced variants of survivin have been reported to interact with BCL2 and inhibit caspase 3 and BCL2-associated X protein (BAX) activity [218]. These proliferative and cytoprotective capacities of survivin make it a strong inducer of tumor cell survival in a post-PDT environment.

TNF- $\alpha$ In addition to activating the NF- $\mathrm{KB}$ response that stimulates survival, TNF- $\alpha$ is known as a potent trigger of apoptosis via the extrinsic pathway as well as necrosis via programmed necrosis or necroptosis. When it binds TNF- $\alpha$, TNFR1 homodimerizes and recruits TRADD and TRAFs to its cytoplasmic domain. In turn, TRADD activates FASassociated with death domain (FADD) and RIP1, which cleaves procaspase 8 to its active form. Subsequently, caspase 8 cleaves BH3 interacting domain death agonist (BID), yielding truncated BID (tBID) that forms a pore in the mitochondrial membrane and allows cytochrome c leakage. Cytochrome $\mathrm{c}$ leakage results in its binding to apoptotic protease activating factor 1 (APAF-1); activation of caspases 9, 3, and 7; and the subsequent activation of the caspase cascade and corollary execution of apoptosis (reviewed in [184]).

Programmed necrosis is the result of RIP1 activation (by e.g., TNF- $\alpha$ ), which forms an autophosphorylating complex with RIP3. This complex activates mixed lineage kinase domain-like protein that interacts with members of the phosphoglycerate mutase family, culminating in the dephosphorylation of dynamin-related protein 1 and the execution of necrosis [184, 219]. The inhibitor of apoptosis proteins (IAPs) constitute the inhibitors of these cell death pathways, which are also upregulated by the NF-kB-TNF- $\alpha$ signaling loop (Section 3.4.2). IAPs have a plethora of functions, and only a brief summary of the most relevant functions is given here. cIAP1/2 act as ubiquitin ligases for RIP1, thereby inhibiting the apoptotic and necroptotic pathways orchestrated by TNF- $\alpha$ while also stimulating RIP1-mediated IKK activation (reviewed in [220]). Additionally, cIAP1/2 is capable of inhibiting the functions of caspases 3, 7, and 9 and therefore of preventing the execution of apoptosis (reviewed in [221]). cIAP $1 / 2$ also inhibits TNF- $\alpha$ signaling by polyubiquitination of NIK and activates JNK and $\mathrm{p} 38^{\mathrm{MAPK}}[222,223]$ to regulate survival, apoptosis, inflammation, and proliferation, which is covered in greater detail in the context of the ASK1 survival pathway (Section 3.4). As stated previously (Section 3.2.2.2 Survivin), survivin is also an IAP family member that inhibits apoptosis and regulates mitosis (reviewed in [145]).

IL-6 One of the most abundant cytokines released by PDTtreated tumor cells is IL-6, which is upregulated by NF- $\mathrm{kB}$ and AP-1 transcription factors [224]. IL-6 functions as a proinflammatory cytokine that binds to the IL-6 receptor (IL-6R) expressed predominantly by immune cells and hepatocytes, or to soluble IL-6R (sIL-6R), which is formed via alternative 
splicing of IL-6R mRNA. The IL-6-IL6R and IL-6/sIL-6R complexes can heterodimerize with glycoprotein 130 (gp130) that is ubiquitously expressed by most cell types, including tumor cells [225]. Stimulated gp130 autophosphorylates its intracellular tyrosine kinase domain [225], leading to activation of Janus kinase proteins and the phosphorylation and subsequent nuclear translocation of STAT3 [226]. Moreover, IL-6 triggers proliferation by activating the RAS-MAPK and PI3K-protein kinase B pathways, resulting in the expression of WNT and COX-2 [226]. Via these pathways, IL-6 trans-signaling induces the epithelial-mesenchymal transition of tumor cells that promotes invasion, metastasis, and disease progression [227-229]. STAT3 is regarded as the main effector of IL-6 signaling and plays an important role in the survival and proliferation of tumor cells and immune cells [230]. Moreover, STAT3 enhances angiogenic signaling and regulates the production of chemoattractants for neutrophils and macrophages [231]. Upon dimerization, STAT3 binds to interferon (IFN) $\gamma$-activated sequence elements to promote survival by upregulating $B C L 2 L 1$, myeloid leukemia cell differentiation protein (MCL1), BIRC4, and BIRC5 (survivin) while downregulating TP53 [231]. Survival is additionally stimulated through upregulation of HSP70, regenerating islet-derived protein III $\beta$ and $\gamma$, trefoil factor 3 , as well as the antioxidant enzymes Mn-SOD, ferritin, and catalase (reviewed in [231]). Proliferation is induced via STAT3 by upregulation of c-JUN, c-FOS, c-MYC, as well as cyclins D and B that mediate cell cycle progression through the G1/S and S/G2 phases, respectively. STAT3 also promotes angiogenesis by facilitating the production of VEGF, HIF- $1 \alpha$, and basic FGF. Besides its role in tumor (re)growth, STAT3 also prompts the immune system by assisting in the production of a wide array of proinflammatory cytokines and chemokines that includes, but is not limited to, CCL2, CXCL2, IL-1 $\beta$, IL- $1 \alpha$, TNF- $\alpha$, and IFN $\gamma$ (the role of STAT3 in conjunction with NF- $\mathrm{kB}$ is comprehensively reviewed in [231]).

Matrix metalloproteinases Remodeling of the tumor microenvironment is essential for cancer progression, and NF- $\mathrm{KB}$ stimulates the expression of enzymes that facilitate extracellular matrix remodeling. MMPs are a family of proteins that cleave matrix peptides to facilitate extracellular matrix remodeling, cell migration, and angiogenesis [232]. These proteins are abundantly expressed by tumor cells, tumor-associated fibroblasts, endothelial cells, and tumor-infiltrated immune cells [233]. MMPs also act as signaling molecules that inhibit apoptosis [232]. By contrast, MMPs have been associated with reduced angiogenesis due to the generation of the antiangiogenic compounds angiostatin and endostatin during the degradation of plasminogen (MMPs 2, 3, 7, 9, and 12) and collagen XVIII (MMPs 3, 9, 12, 13, and 20), respectively [234]. The exact role of MMPs in tumor biology and responsiveness to PDT is currently elusive and deserves further context-dependent investigation.

\subsubsection{Role of the NF- $\kappa B$ pathway in PDT}

NF- $\mathrm{KB}$ is one of the major transcription factors induced by PDT [194, 195, 235-239], although in some instances NF-kB was also found to be downregulated following PDT, such as in nasopharyngeal carcinoma (hypericin as photosensitizer) and breast cancer cell lines (C-phycocyanin as photosensitizer) [240, 241]. Despite the elusive NF-KB activation mechanism(s) in case of PDT, it is clear that NF-KB activation does occur after PDT on the basis of findings concerning at least two downstream targets of the NF- $\mathrm{KB}$ transcription factor, namely COX-2 and survivin. COX-2 mRNA and protein levels as well as COX-2 activity were increased after PDT in a multitude of studies [202, 239, 242-246], albeit COX-2 activity was not necessarily attributed to NF- $\mathrm{KB}$ activation [247] but rather to IL- 6 or $\mathrm{p} 38^{\mathrm{MAPK}}$ signaling [243, 244, 248]. Similarly, survivin (Section 3.2.2.2 Survivin) was upregulated and phosphorylated after PDT in a number of studies [249-253]. This upregulation was most likely mediated by E2F and STAT3 transcription factors [254], which are indirectly activated by PDT through growth factors (e.g., epidermal growth factor (upregulated via the ASK1-AP-1 pathway, Section 3.4.2.2 Prolonged downstream effects of ASK1 activation) and VEGF) and cytokines (IL-6) downstream of the HIF-1 and NF-KB pathways (Section 3.2.2). IL-6 functions as a survival factor and also as a regulator of the antitumor immune response after PDT by activating STAT3 and COX-2. Presently, it is unclear whether inhibition of IL- 6 signaling by for example blocking AP-1 and/or NF-KB is beneficial or detrimental to tumor response. Several studies have explored the function of IL-6 following PDT, but the investigations have yielded contradictory results. First, expression levels of IL-6 vary depending on the cell line, at least in case of nasopharyngeal cancer cell lines. Whereas CNE-2 cells showed a 13-fold increase in IL-6 mRNA levels compared to untreated cells, HK-1 cells exhibited only a 1.4-fold increase in IL-6 mRNA levels $6 \mathrm{~h}$ post-PDT. The effect of IL- 6 overexpression on the response to PDT was not investigated [255]. Secondly, the outcomes regarding the prosurvival or prodeath role of IL6 are conflicting. On the one hand, IL-6 stimulated tumor cell survival and negatively regulated the antitumor immune response in mice bearing Colo26 xenografts [256]. Similarly, IL-6 induction by PDT was associated with cell death inhibition and enhanced tumor growth in human basal cell carcinoma (BCC-1/KMC) cells [247] and mice bearing subdermal Co26 murine colon carcinomas or 4T1 mammary carcinomas [256]. On the other hand, a beneficial effect of IL-6 overexpression for PDT has been reported. Tumor growth in mice was reduced by IL-6 in human prostate cancer (LnCAP) xenografts [257] and human neuroblastoma (WAC2) xenografts 
[258]. Similarly, mice bearing Lewis lung carcinomas were more susceptible to PDT when the cells overexpressed IL-6 [259]. In a clinical setting, high levels of IL-6 following PDT of cholangiocarcinomas correlated positively with increased tumor mass, indicating that elevated IL-6 levels enhance tumor growth and/or recurrence following PDT [260]. With respect to transcriptional regulation of MMPs after PDT, the AP-1 transcription factors FOS and ATF2 that are activated in the ASK1 survival pathways (Section 3.4.2.1 Acute downstreameffects of ASK1 activation) as well as NF-KB are able to upregulate the expression of MMP1, MMP2, MMP3 [261, 262], and MMP9 [263]. However, the regulation of MMPs following PDT is ambivalent. For example, MMPs 1-3 were upregulated or activated in HK-1 nasopharyngeal carcinoma cells, MCF-7 mammary carcinoma cells and xenografts, in a Walker carcinosarcoma model, and in keratinocyte-associated fibroblasts that were subjected to PDT [240, 264-266]. Furthermore, long-term upregulation of MMP9 but not MMP1, MMP3, MMP7, and MMP12 was observed in actinic keratosis patients treated by PDT [267]. Conversely, several studies have reported the downregulation of MMPs after PDT, including MMP2 and MMP9 in human nasopharyngeal carcinoma, oral cancer, medulloblastoma, and glioma cell lines (HK-1; UP and VB6; MED TE-671; and U87 and GBM6840 cells, respectively) [240, 268-270]. The reduction in MMP2 and MMP9 levels was associated with retarded tumor cell migration $[268,269]$. Furthermore, human glioma spheroids treated with PDT exhibited reduced MMP7 and MMP8 levels, a depolarized morphology, and a reduced migration and invasion capacity compared to untreated spheroids [271].

\subsubsection{Inhibition strategies for $N F-\kappa B$ and its downstream targets}

Inhibition of NF- $\mathbf{k B}$ Terpenoids are NF- $\mathrm{kB}$ inhibitors with a variety of structures and various modes of action [272] (Table 1). The sesquiterpene parthenolide has proven particularly useful for promoting the alkylation of Cys38 in the RelA subunit of $\mathrm{NF}-\mathrm{kB}$, thereby preventing DNA binding [137, 272]. Alternatively, panepoxydone is an inhibitor of NF- $\mathrm{kB}$ that blocks phosphorylation of IKB by TNF- $\alpha$ without coincident activation of AP-1 in COS-7 monkey kidney cells [138]. However, neither parthenolide nor panepoxydone has been employed in studies combined with PDT. The small molecule Bay 11-7082 is frequently used as a chemical inhibitor of IKK to prevent NF-KB activation both in vitro and in vivo. This compound effectively inhibited TNF- $\alpha$-mediated phosphorylation and activation of IKK in lipopolysaccharide-treated murine RAW264.7 macrophages, which translated to impaired phosphorylation and cytosolic retention of NF-KB [139]. However, as shown by Lee et al., the main target of Bay 11-7082 does not appear to be IKK since it also inhibits AKT phosphorylation upstream of IKK activation in the TNF- $\alpha$ signaling pathway [139]. The activity of c-FOS and c-JUN was also diminished by Bay 11-7082 in COS-7 cells
[139]. Thus, the inhibitory mechanism of Bay 11-7082 on $\mathrm{NF}-\mathrm{KB}$ stems from its action at sites upstream of IKK rather than direct modulation of NF-KB activity (Table 1).

The precise mechanism notwithstanding, Coupienne et al. demonstrated that LN18 human glioblastoma cells were significantly more sensitive to ALA-PDT following incubation with $10 \mu \mathrm{M}$ of Bay 11-7082 for $30 \mathrm{~min}$ prior to PDT [273]. Contrastingly, we have recently demonstrated that siRNA-mediated inhibition of the RelA subunit of NF- $\mathrm{KB}$ resulted in reduced susceptibility of murine mammary carcinoma (EMT-6) cells to ZnPC-PDT. While cell viability post-PDT increased in RelA-inhibited cells, the PDT-treated cells released increased levels of TNF- $\alpha$, CCL2, and IL-6. Accordingly, the supernatant isolated from these cells exerted enhanced immunogenicity on RAW264.7 murine macrophages [274]. In agreement with these results, Chen et al. found that Bay 11-7082 and also SP600125, an inhibitor of JNK (Table 1), greatly reduced the amount of apoptotic human Ca9-22 oral cancer cells following ALA-PDT, suggesting that $\mathrm{NF}-\mathrm{KB}$ and JNK jointly regulate apoptotic signaling following PDT [275]. However, given the multitude of inhibitory effects of Bay 11-7082, it is difficult to ascertain whether the increased or reduced sensitization to PDT is the result of NF-KB inhibition or of impaired AP-1 activity. Rapozzi et al. reported that pheophorbide A-PDT in combination with the NF- $\mathrm{KB}$ inhibitor dehydroxymethylepoxyquinomicin (DHMEQ, Table 1) [140] promoted cell death in B78-H1 murine amelanotic melanoma cells compared to PDT without DHMEQ [276], which is in support of PDT-induced NF-KBmediated survival signaling.

Since NF-kB and HIF-1 share a similar activation mechanism following PDT (Sections 3.2.2 and 3.3.2), $\alpha$ ketoglutarate may serve as an inhibitor of both signaling cascades (Table 1). PHD1 and 3 lose their HIF-1 and NF-KB inhibitory capacity under hypoxic conditions, but the activity of PHDs can be restored by increasing intracellular $\alpha$ ketoglutarate levels, even under low oxygen tensions [277]. Moreover, the activation pathways of NF-kB and HIF-1 are highly interconnected due to transcriptional upregulation of HIF- $1 \alpha$ mRNA by NF-kB and also the HIF-1-mediated production of cytokines, such as TNF- $\alpha$, that can activate NF-kB. Since hypoxia does not play a major role in the activation of NF-KB [168], NF-KB activation is more likely to result from TNF- $\alpha$ production downstream of the HIF-1 and AP-1 pathways. However, studies in our lab with liposomal zinc phthalocyanine-PDT have shown that incubation of tumor cells with free or liposome-delivered $\alpha$-ketoglutarate does not enhance PDT efficacy (Broekgaarden, M. et al., Nano Research, in resubmission; Weijer, R. et al., Oncotarget, in resubmission), which is further discussed in Section 3.3.4.

Inhibition of COX-2 COX-2 is an important regulator of post-PDT survival [278] insofar as inhibition of COX-2 prior 
or during PDT has consistently yielded increased tumor cell death after PDT [242, 244, 245, 251, 279-281]. Since COX-2 is under the control of both NF-KB and ATF2, inhibition of NF-kB (with, e.g., Bay 11-7085) and also $\mathrm{p} 38 \alpha$ (with, e.g., PD169316, SB202190, or SB203580, Table 1) indeed reduced COX-2 protein levels and increased the responsiveness to PDT in human ovarian (HeLa) and bladder cancer (T24) cells as well as radiation-induced mouse fibrosarcoma (RIF-1) cells [202, 239, 244]. In addition, suppression of the AP-1 activators protein kinase $\mathrm{C}$ (PKC) and MKK1 and 2 led to decreased COX-2 levels in hypericin-PDT-treated T24 cells and porfimer sodium-PDT-treated RIF-1 cells [202, 239]. These results further attest to the importance of the AP-1 and NF- $\mathrm{kB}$ signaling pathways in terms of COX-2 activation and the survival response that ensues after PDT. The most commonly used COX inhibitors are nonsteroidal antiinflammatory drugs (NSAIDs), which bind to Arg120 of COX-1 and COX-2 to subsequently block the conversion of arachidonic acid to $\mathrm{PGH}_{2}[142,282,283]$ (Table 1). Some NSAIDs bind only to COX-1 (e.g., flurbiprofen), whereas others bind to both COX-1 and COX-2 (e.g., naproxen, indomethacin, ibuprofen, and aspirin) [284] or inhibit COX-2 directly, including celecoxib, rofecoxib, nimesulide, diclofenac, meloxicam, and the related compound NS-398 [142, 284, 285]. The latter two groups of inhibitors are suitable for use in PDT because they target COX-2. Accordingly, inhibition of COX-2 prior or during PDT with NSAIDs decreased tumor cell survival in a variety of (tumor) cell lines [242, 245, 251, 279-281, 286], which coincided with a reduction in levels of $\mathrm{PGE}_{2}[244,280]$ and the proangiogenic factors MMP9, TNF- $\alpha$, IL-1 $\beta$, IL-10, and VEGF [280]. Moreover, inhibition of COX-2 with NSAIDs caused a reduction in the levels of the antiapoptotic protein survivin [251].

One of the concerns of inhibiting COX-2 activity is that the consequent reduction in cytokine production may abolish the antitumor immune response necessary for long-term protection against tumor recurrence [169] and removal of residual or non-PDT damaged tumor cells in immunocompetent hosts $[83,84]$. However, blocking of COX-2 with celecoxib, NS398 , or nimesulide showed considerably increased survival of immunodeficient mice in which various tumor cell lines were xenografted $[242,245,251,280]$. Thus, the inhibition of COX-2 activity with NSAIDs could be a valuable intervention strategy for PDT to reduce tumor cell survival and potentially reduce the proangiogenic effects induced by $\mathrm{PGE}_{2}$.

Inhibition of survivin Inhibition of survivin, which is upregulated by activation of NF-KB following PDT (Section 3.2.2.2 Survivin), may reduce antiapoptotic signaling and therefore could result in increased PDT efficacy. Several different compounds that inhibit survivin are available that either block upstream activators such as HSP90 (17-AAG) and STAT3 (STA-21 [143] or WP1066 [144]) or inhibit survivin directly via antisense RNA interference (LY218130B) and/or transcriptional repression (YM155 and EM1421) [145] (Table 1), although the specificity of the latter compounds may not be restricted to survivin [287].

Some investigations studying the inhibition of survivin during PDT have employed geldanamycin (17-AAG) to inhibit HSP90-induced survivin expression [250, 252], celecoxib or 2,5-dimethyl celecoxib [251] for direct inhibition of survivin (although the mechanism by which these compounds inhibit survivin remains elusive), or have applied gene knockdown strategies [249]. Regardless of the inhibition strategy, all these studies point toward an increased tumoricidal effect of survivin inhibition during PDT, making survivin an important target for PDT enhancement strategies.

Inhibition of IL-6 Unequivocal evidence for the prosurvival role of IL-6 in PDT-subjected tumor cells is lacking since both beneficial and detrimental effects of IL-6 signaling in terms of cell survival have been observed after PDT (Section 3.2.2.4 IL-6). Although the use of IL-6 inhibitors has not been explored in PDT research, cancer-related studies in which IL-6 signaling was inhibited may provide clues as to the potential (neo)adjuvant efficacy of IL-6 inhibitors for the enhancement of PDT. A specific blocker of IL-6/sIL-6R transactivation has been developed by fusing the extracellular domain of human gp130 to a human IgG1 antibody (sgp130Fc, Table 1). The molecule was shown to effectively block IL-6 signaling in mouse and rat models of autoimmune disease (reviewed in [146] and [288]). For example, sgp130Fc significantly prevented disease progression in inflammation-associated mouse cancer models. Thus, blocking of IL-6 transactivation with sgp $130 \mathrm{Fc}$ after PDT could increase the therapeutic potential and may be instrumental in elucidating the role of the IL-6 signaling pathway in tumor cell survival.

Inhibition of matrix metalloproteinases Inhibitors for MMPs are readily available, and most agents inhibit multiple MMP isoforms. This is particularly important since the antitumor effects of MMP inhibitors are not confined to a single isozyme but are mediated by, e.g., MMP3, MMP8, and MMP12 [232]. Ferrario et al. investigated the broad spectrum MMP inhibitor prinomastat [147] (Table 1) in combination with PDT in mouse BA mammary carcinoma xenografts [289] after observing increased levels of MMP2 and MMP9 expression after porfimer sodium-PDT. Long-term cures were found in $46 \%$ of mice treated with prinomastat and PDT versus only $20 \%$ in mice treated with PDT alone, although the enzymatic activity in the presence of prinomastat was not assayed. Accordingly, the inhibition of MMPs during PDT holds potential for the enhancement of therapeutic efficacy. Despite the positive results, caution should be exercised when designing an MMP inhibitor-based 
combinatorial treatment in light of the variable regulation of different MMP isozymes and their ambivalent biological effects (tumor suppressing and tumor promoting). For example, wound healing relies on MMPs, and it is possible that pharmacological inhibition may interfere with the recovery of PDT-treated tissues.

\subsubsection{Concluding remarks}

The contribution of NF- $\mathrm{kB}$ to the cell survival response appears to be well-established based on the studies that have demonstrated NF-KB activation following PDT (Section 3.2.3). At least three possible mechanisms are responsible for NF- $\mathrm{kB}$ activation after PDT (Section 3.2.1) and pharmacological interventions in the NF- $\mathrm{kB}$ survival pathway are possible to improve PDT outcomes (Section 3.2.4). However, such interventions may present a therapeutic quagmire. On the one hand, the downstream targets of NF- $\mathrm{kB}$ are instrumental for tumor cell survival following PDT, such as COX-2 and survivin, of which the inhibition results in increased tumor cell death and better tumor control (sections 3.2.4.2 Inhibition of COX-2 and 3.2.4.3 Inhibition of survivin). On the other hand, many proinflammatory cytokines are upregulated by NF-KB that can attract cells of the innate and adaptive immune system to mediate an antitumor immune response. Interfering with the capability of the treated cancer cells to produce a variety of cytokines and chemokines may therefore inhibit the antitumor immune response and reduce long-term therapeutic efficacy. In contrast to the postulations, it was recently shown that inhibition of NF- $\mathrm{KB}$ resulted in increased cytokine release and immunogenicity of PDT-treated tumor cells in vitro [274] and suggested that NF-KB may not be a suitable target for pharmacological inhibition in conjunction with PDT. These contrasting results demonstrate that further research on the in vitro and in vitro consequences is pivotal to understand the complex functions of NF-kB in a post-PDT tumor microenvironment.

\subsection{The HIF-1 pathway}

Tumor growth frequently leads to hypoxia since the tumor tissue tends to outgrow its immature blood supply, as a result of which a hypoxia-induced inflammatory response is triggered to stimulate angiogenesis and increase metastasis. Tumor cells cope with mildly hypoxic conditions by constitutively activating HIF-1, leading to the transcription of genes involved in anaerobic metabolism, inflammation, and antioxidant responses [290]. Under conditions of acute severe hypoxia or anoxia, tumor cells hyperactivate HIF-1 and its downstream responses for purposes of survival (Broekgaarden, M. et al., Nano Research, in resubmission; Weijer, R. et al., Oncotarget, in resubmission). HIF-1 activation has been observed in many PDT studies, and HIF-1 has been accepted as one of the main molecular effectors induced by PDT [246, 250, 291-294]. The remainder of this section will address the four main activation mechanisms of HIF-1 (Section 3.3.1) and the most important downstream effects that may play a role in tumor cell survival post-PDT (Section 3.3.2). Evidence for its activation after PDT is addressed in Section 3.3.3, and the potential HIF-1 intervention strategies to enhance PDT efficacy are discussed in Section 3.3.4.

\subsubsection{Activation mechanisms of HIF-1}

The HIF-1 transcription factor is a basic helix-loop-helix (bHLH) heterodimeric protein composed of an $\alpha$ subunit (HIF- $1 \alpha$ or HIF- $2 \alpha$ ) and a $\beta$ subunit (HIF-1 $\beta$ ) subunit [295]. HIF- $1 \alpha$ is constantly transcribed but retained in the cytosol and rapidly degraded under normophysiological conditions. HIF-1 $\beta$ is constitutively expressed in the nucleus, where it is separated from its dimerization partner HIF- $1 \alpha$ in the cytosol and thus kept inactive. Upon stabilization, HIF-1 $\alpha$ translocates to the nucleus, dimerizes with HIF- $1 \beta$, and binds DNA at hypoxia responsive elements (HREs) to initiate target gene expression [296, 297]. The effects of HIF-1 activation are profound, since over 500 genes are known to be a direct target of HIF-1. Moreover, HIF-1 is involved in chromatin remodeling complexes and microRNA expression that regulate gene expression at an epigenetic level [298-301]. There are at least four different mechanisms by which HIF-1 $\alpha$ may become activated after PDT, namely hypoxia, ROS, NF-kB, and COX-2. The pathways are summarized in Fig. 5.

HIF-1 activation by hypoxia HIF- $1 \alpha$ acts as an oxygen sensor in that it is constantly targeted for proteasomal degradation under normoxic conditions as a result of hydroxylation and subsequent polyubiquitination [295, 297, 302-305]. Hydroxylation of HIF- $1 \alpha$ by PHD2/ 3 and FIH leads to HIF- $1 \alpha$ recognition and binding by VHL proteins, which act as a scaffold for E3 ubiquitin ligase that polyubiquitinates HIF- $1 \alpha$ as a signal for proteasomal degradation [306, 307]. During hypoxia, which occurs after PDT (Section 2.2.2), HIF-1 $\alpha$ hydroxylation by PHDs and FIH ceases because the hydroxylation reaction requires $\mathrm{O}_{2}$ [308]. This causes HIF- $1 \alpha$ to become stabilized, move to the nucleus, complex with HIF- $1 \beta$, and activate gene transcription through HREs.

HIF-1 activation by ROS HIF- $1 \alpha$ stabilization by hypoxiamediated PHD and FIH inactivation can also proceed through ROS-mediated deactivation of PHDs and FIH in a manner that is not necessarily dependent on intracellular oxygen tension [309-311]. PHDs and FIH require $\mathrm{Fe}^{2+}$ as cofactor in their conversion of $\alpha$-ketoglutarate, $\mathrm{O}_{2}$, and proline to succinate, $\mathrm{CO}_{2}$, and hydroxyproline, respectively. It should be noted that succinate is an important electron donor in the citric acid cycle [312]. Oxygen radicals, which are abundantly produced during PDT (Section 2.2.1), are able to oxidize $\mathrm{Fe}^{2+}$ to $\mathrm{Fe}^{3+}$, thereby inhibiting the enzymatic activity of PHDs and FIH 


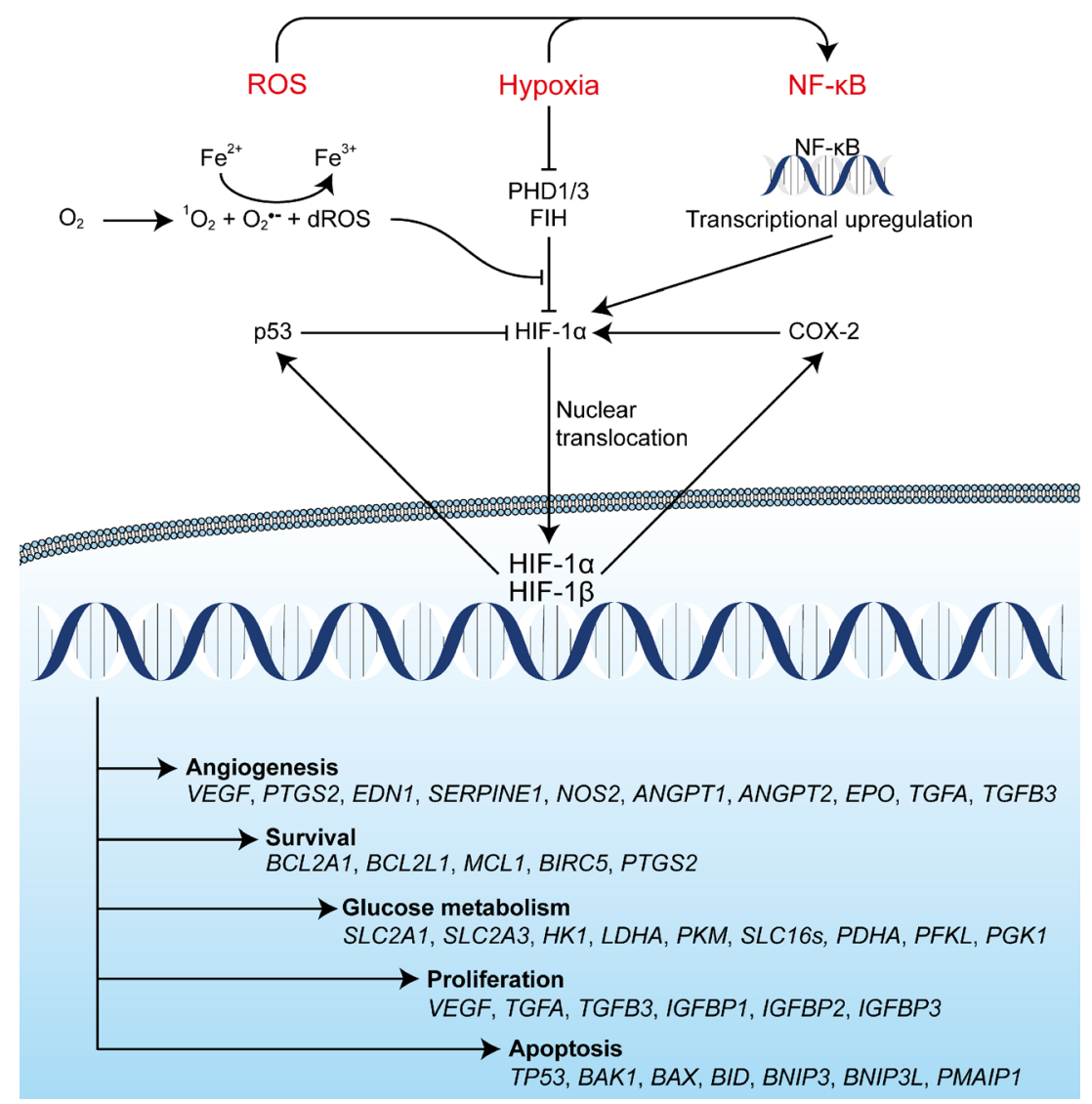

Fig. 5 Activation of HIF- $1 \alpha$ after PDT is mediated by several pathways. PDT-induced hypoxia due to immediate $\mathrm{O}_{2}$ depletion as well as vascular shutdown prevents HIF- $1 \alpha$ hydroxylation by PHDs and FIH, which is an $\mathrm{O}_{2}$-dependent process. Furthermore, ROS-mediated oxidation of $\mathrm{Fe}^{2+}$ in the catalytic center of PHDs and FIH disables the enzymatic activity of these proteins. Both events lead to HIF-1 $\alpha$ stabilization, translocation from the cytosol to the nucleus, complexation with HIF-1 $\beta$, and transcriptional upregulation of numerous target genes containing an HRE in the promoter region. HIF- $1 \alpha$ transcription is also upregulated by PDT-activated NF- $\mathrm{KB}$, which increases $\mathrm{HIF}-1 \alpha$ protein levels. A

and reducing hydroxylation-mediated degradation of HIF- $1 \alpha$, even under normoxic conditions [313]. The ROS-driven activation of HIF-1 may also be significant under hypoxic conditions. Hypoxia induces mitochondrial ROS release that was shown to be responsible for an initial burst of HIF-1 activation, whereas hypoxia-driven, NADPH-oxidase-induced ROS induced a second burst of HIF-1 activation in human HS29-4T melanoma cells [314]. Thus, the activation mechanisms of HIF-1 by ROS and hypoxia appear to be interrelated.

HIF-1 activation by NF- $\kappa$ B In addition to modulation by ROS and hypoxia, HIF- $1 \alpha$ expression is at least partially under the control of NF-KB since the promoter of the HIF $1 A$ gene contains an NF-kB binding site [197]. Cultured pulmonary artery smooth muscle cells incubated with $\mathrm{H}_{2} \mathrm{O}_{2}$ under normoxic conditions exhibited NF- $\mathrm{kB}$ activation that was responsible for transcriptional upregulation of HIF-1 $\alpha$ and the HIF-1 target gene plasminogen activator inhibitor 1 (PAI1) negative feedback loop for HIF-1 $\alpha$ exists via the upregulation of p53 by activated HIF-1, which targets HIF-1 $\alpha$ for proteasomal degradation in the presence of DNA damage. A positive feedback loop exists via the upregulation of COX-2 by activated HIF-1 and NF- $\mathrm{KB}$. COX-2 is involved in the production of $\mathrm{PGE}_{2}$ that plays a role in the transactivation of HIF-1 $\alpha$. After nuclear translocation, dimerization, and DNA binding to HRE sequences, HIF-1 transcription factors facilitate the upregulation of a plethora of genes involved in angiogenesis, survival, glucose metabolism, proliferation, and apoptosis. Other pathways are affected as well, but only those most relevant for PDT are depicted

[315]. Given that NF- $\mathrm{BB}$ is activated by inflammation, hypoxia, and ROS (Section 3.4.1), transcriptional upregulation of HIF $1 A$ by NF-KB and subsequent translation to a functional unit may be one of the most important regulatory mechanisms that influences HIF-1 signaling.

HIF-1 activation by COX-2 The intricate relationship between NF-KB and HIF-1 signaling is further exemplified through $\mathrm{COX}-2$. In addition to transcriptional regulation by NF-KB, the PTGS2 gene that encodes COX-2 contains four HRE sequences in its promoter region, explaining its upregulation by HIF-1 under hypoxic or prooxidative conditions [201]. Interestingly, COX-2 amplifies the HIF-1 pathway. COX-2 facilitates the production of $\mathrm{PGE}_{2}$ (Section 3.2.2.1 COX-2), which can subsequently stimulate the transcriptional activity of the HIF-1 complex [201]. As such, there is a positive feedback loop for COX-2 and HIF-1 activity, although the exact molecular interactions have not been fully elucidated. 


\subsubsection{Downstream effects of the HIF-1 pathway}

When the growth of the tumor parenchyma is more extensive than the formation of new blood vessels, the deficiency in oxygen supply will trigger HIF-1 activation that in turn signals a metabolic switch to glycolysis and a consequent decrease in oxygen demand. The consequent decrease in $\mathrm{O}_{2}$ consumption expands the area of $\mathrm{O}_{2}$ availability within the tumor, allowing distally situated tumor cells (relative to the vasculature) to proliferate, which benefits tumor growth as a whole [297]. Inasmuch as persistent hypoxia can only be resolved by the formation of new blood vessels, HIF-1 signaling is programmed to stimulate angiogenesis [316] (Fig. 5). The vascularization of a tumor requires degradation of the extracellular matrix to enable vessel sprouting, migration, and maturation of mesenchymal cells into endothelial cells; tube formation; and pericyte recruitment to endothelialize the newly formed lumens (reviewed in [317]). Therefore, a hypoxic tumor microenvironment and the HIF-1 transcription factor are important mediators of cell survival and tumor regrowth following therapy.

With respect to glucose metabolism, tumor cells and tumorassociated cells become less dependent on oxygen during hypoxia by reducing oxidative phosphorylation and increasing anaerobic respiration (i.e., glycolysis; Warburg effect) [318]. HIF-1 is instrumental in this transformation by initiating the transcription of genes involved in glucose metabolism. The target gene products include glucose transferases 1 and 3 (GLUT1/3, SLC1A1/3), hexokinase (HK, HK1), lactate dehydrogenase A (LDHA), monocarboxylate transporters (MCTs, SLC16As), pyruvate dehydrogenase (PDH), pyruvate kinase (PKM), phosphofructokinase L (PFKL), and phosphoglycerate kinase I (PGK1) (reviewed in [297] and [296]) (Fig. 5). Despite the prevailing state of hyponutrition as a result of PDT-induced vascular shutdown, residual viable tumor cells may scavenge glucose from the tumor microenvironment to support anaerobic respiration. This glucose may have been released from tumor cells immediately killed by PDT to support anaerobic respiration. Intratumoral angiogenesis, endothelial cell proliferation, and matrix and vascular remodeling are modulated by HIF-1 via upregulation of VEGF, endothelin 1 (EDN1), plasminogen activator inhibitor 1 (PAI1, SERPINE1), (inducible) nitric oxide synthase 2 (NOS2), angiopoietin $(A N G P T) 1$ and 2, erythropoietin $(E P O)$, and transforming growth factor (TGF)- $\beta 3$ (TGFB3) [299, 319] (Fig. 5). Proliferation of tumor and tumor-associated cells is stimulated by HIF-1 through the induction of genes encoding insulin-like growth factor (IGF) 2 as well as IGF binding proteins 1 , 2, and 3; TGF- $\alpha$ and TGF- $\beta 3$; and VEGF [296, 297] (Fig. 5). In this process, COX-2, which is a target gene of HIF-1 (Section 3.3.1.4 HIF-1 activation by COX-2), orchestrates a positive feedback loop that reinforces the activity of both COX-2 and HIF-1 [201] (Fig. 5). PGE 2 is produced by COX-2 and enhances HIF1A transcription and induction of
HIF-1, which subsequently binds the COX-2 promoter to upregulate its expression [201]. Taken altogether, HIF-1 potentiates numerous critical biological responses to PDT that revolve around tumor cell survival and enables cells to cope with and recover from the damage caused by PDT. Lastly, HIF-1 has been shown to have notable effects on cell death pathways. In addition to transcriptionally upregulating survivin (BIRC5) (Section 3.2.2.2 Survivin) and HO-1 (Section 3.1.2), HIF-1 regulates prosurvival proteins of the BCL2 family (BCL2 (BCL2A1), BCL-XL (BCL2L1), BID, and MCL-1 (MCL1)) (Fig. 5), although proapoptotic members of the same family have also been reported to be upregulated by HIF-1, including BCL2-homologous antagonist killer (BAK), BAX, BCL2/adenovirus E1B $19 \mathrm{kDa}$ protein-interacting protein 3 (BNIP3), BNIP3 ligand (BNIP3L), and NOXA (phorbol-12-myristate-13-acetate-induced protein 1, PMAIP1) [320]. However, HIF-1-mediated induction of BNIP3 and BNIP3L may also be essential for hypoxia-driven cytoprotective autophagy and facilitate hypoxic survival, at least in human prostate cancer (PC-3) cells [321]. Furthermore, HIF-1 has been implicated in the stabilization of tumor suppressor protein $\mathrm{p} 53$ [322], which promotes apoptosis upon oncogenic stress and negatively regulates HIF-1 $\alpha$ stability (Fig. 5) [323]. Although HIF-1 predominantly stimulates survival through various biological processes, proapoptotic signaling may also occur in the presence of DNA damage via p53-mediated activation of proapoptotic BCL2-family members that are upregulated by HIF-1.

\subsubsection{Role of the HIF-1 pathway in PDT}

Although HIF-1 is considered an important transcription factor in the context of PDT [17], very few studies have investigated HIF1 activity following PDT. Chemical induction of HIF-1 by preincubating human Het-1 esophageal cells with $500 \mu \mathrm{M} \mathrm{CoCl}$ desensitized cells to ALA-PDT [324]. Mitra et al. demonstrated that HIF-1 is activated by porfimer sodium-PDT in murine breast cancer (EMT-6) cells transfected with a gene encoding green fluorescent protein (GFP) under the control of a promoter sequence with five HREs [293]. The expression of GFP after PDT occurred under normoxic conditions, underscoring the relevance of ROS-mediated activation of HIF-1 in the absence of hypoxia (Sections 3.3.1.2 HIF-1 activation by ROS and 3.3.1.3 HIF-1 activation by NF-kB). The authors argued that $\mathrm{PGE}_{2}$ synthesized by COX-2 (Section 3.3.1.4 HIF-1 activation by COX-2) may be an important mediator of HIF-1 activity, although no corroborative evidence was obtained in COX-2 inhibition experiments [293]. The technical difficulties in studying HIF-1 in an in vitro PDT setting result from the requirement for hypoxic culture conditions and the short half-life of HIF- $1 \alpha$ under normoxic conditions (5-8 $\mathrm{min}$ ) [325]. Despite these difficulties, Krieg et al. showed increased HIF-1 $\alpha$ protein expression following ALA-PDT in UROtsa, RT112, and J82 (but not RT4) human 
bladder cancer cells under normoxic conditions using reversed phase protein arrays [292]. Stabilization and activation of HIF-1 under hypoxic conditions was recently demonstrated in human epidermoid carcinoma (A431) and human extrahepatic cholangiocarcinoma (Sk-Cha1) cells after PDT with liposomal zinc phthalocyanine. In line with HIF- $1 \alpha$ stabilization, VEGF, $P T G S 2$, and HMOX-1 mRNA were upregulated to a greater extent after PDT than in untreated hypoxic cells (Broekgaarden, M. et al., Nano Research, in resubmission; Weijer, R. et al., Oncotarget, in resubmission). Additional evidence for the prominent role of HIF-1 in PDT was provided in a mouse model of Kaposi's sarcoma using porfimer sodium-PDT. Tumors collected $1 \mathrm{~h}$ after PDT exhibited increased HIF-1 $\alpha$ protein levels compared to untreated tumors. The HIF- $1 \alpha$ protein levels in PDTtreated tumors were comparable to those in tumors of which the blood supply had been clamped for $30 \mathrm{~min}$ [326]. Similar results regarding HIF-1 activation were obtained in human nasopharyngeal carcinoma (CNE-2) xenografts in mice that had been subjected to hypericin-PDT [246] and in rat chorioretinal tissue treated with verteporfin-PDT [294]. The increased mRNA expression and protein levels of HIF-1 $\alpha$ were associated with increased protein levels of VEGF, as was demonstrated in a murine model of mammary (BA) carcinoma treated with porfimer sodium-PDT [291], indicating that post-PDT HIF-1 signaling induces angiogenic remodeling of the affected tissue (Section 3.3.2 and Fig. 5). Moreover, a clinical study by Koukourakis et al. revealed that esophageal tumors with high intratumoral protein levels of HIF-1 were more resistant to PDT compared to tumors with low HIF-1 protein levels [327], attesting to the involvement of HIF-1mediated survival pathways following PDT (Section 3.3.2 and Fig. 5). Increased HIF-1 $\alpha$ protein levels were also observed in mouse porfimer sodium-PDT-treated murine BA mammary carcinoma tumors, but this was not reported for porfimer sodiumPDT-treated BA cells in vitro [250].

\subsubsection{Inhibition strategies for HIF-1 and its downstream targets}

Due to the importance of HIF-1 in tumor survival, therapeutic interventions for cancer encompass the inhibition of HIF-1 [290]. However, most HIF-1 inhibitors are rather unspecific and also target the upstream modulators of HIF- $1 \alpha$ protein synthesis, of which imatinib (an inhibitor of breakpoint cluster region protein (BCR)-ABL [328]), gefitinib, erlotinib, and cetuximab (an inhibitor of EGFR [329]), and everolimus (an inhibitor of mTOR [330]) are well-known examples [290] (Table 1). Another combination strategy is to interfere with the stabilization of HIF-1 by inhibition of chaperone binding using geldanamycin (an inhibitor of HSP90 [331]) or increasing the affinity for natural inhibitors of HIF-1 (e.g., amphothericin B [148]) (Table 1). Interfering with HIF-1 DNA binding is another approach to reduce HIF-1 signaling. For example, echinomycin competes with HIF-1 to bind to HREs and can therefore be used to reduce transcriptional activity of HIF-1 [149] (Table 1). As mentioned previously, these inhibitors are rather unspecific, which may be valuable in the development of a combinatorial cancer therapy. However, a more specific inhibitor of HIF-1 would be desirable when investigating the mechanism of HIF-1 on tumor cell survival following PDT.

$\alpha$-Ketoglutarate may be a useful drug as a specific inhibitor of HIF-1 (Table 1). Under normophysiological conditions, PHDs are the major inhibitors of HIF-1 activity during normoxia but are rendered dysfunctional during hypoxia [332] (Section 3.3.1 and Fig. 5). The endogenous molecule $\alpha$-ketoglutarate is a selective PHD substrate and agonist [312], and it is able to reactivate PHDs to inhibit HIF-1 regardless of intracellular oxygen tension [141]. Under normoxic conditions, PHDs facilitate the conversion of $\alpha$-ketoglutarate and oxygen to succinate and carbon dioxide, respectively, but also transfer oxygen to prolyl residues in the HIF-1 $\alpha$ oxygen-dependent degradation domain (ODD) [312]. Increasing the activity of PHDs after PDT with $\alpha$ ketoglutarate may therefore render cells less susceptible to HIF1-mediated survival. Studies by Mackenzie et al. have shown that, despite hypoxia, the activity of PHD2 and 3 and the concurrent destabilization of HIF-1 in various tumor cell lines and murine xenografts could be induced by the administration of $\alpha$ ketoglutarate esters (esterification allows passage through the membrane into the cell) [141]. The inhibition of HIF-1 by $\alpha$ ketoglutarate was associated with decreased tumor growth and increased apoptosis [277, 333]. Based on these investigations, HIF-1 inhibition by $\alpha$-ketoglutarate may be a valuable strategy in potentiating the effects of PDT. However, recent studies by our group revealed that $\alpha$ ketoglutarate did not increase the efficacy of PDT, but rather reduced PDT-induced oxidative stress as measured $4 \mathrm{~h}$ post-PDT in A431 cells. It was hypothesized that $\alpha$-ketoglutarate was used as an energy source to fuel antioxidant responses or that it functions as an antioxidant itself and thus is not a suitable agent to enhance the PDT response (Broekgaarden, M. et al., Nano Research, in resubmission; Weijer, R. et al., Oncotarget, in resubmission).

Another nonspecific inhibitor of HIF-1 is the naturally occurring diphenolic compound curcumin (Table 1), which was found to promote proteasomal degradation of HIF-1 $\alpha$ and HIF-1 $\beta$ (also known as aryl hydrocarbon receptor nuclear translocator, ARNT) [150, 334, 335]. Moreover, Choi et al. [150] showed that HIF-1 activity was reduced as a result of curcumin-stimulated oxidation and proteasomal degradation of HIF-1 $\beta$. The authors further showed that the inhibition of HIF-1 $\beta$ was dependent on oxidation, as addition of the antioxidant $\mathrm{N}$-acetylcysteine prevented HIF- $1 \beta$ inactivation by curcumin. However, it should be noted that curcumin exerts many other mainly cytostatic/toxic effects on tumor cells, including the inhibition of EGFR tyrosine kinase activity and downstream signaling; inhibition of protein kinase $\mathrm{C}, \mathrm{COX}-2$, and $\mathrm{NF}-\mathrm{kB}$; and induction of DNA 
damage [336]. These effects tend to increase PDT efficacy, as exemplified by the finding that curcumin increases ROS production, mitochondrial membrane permeabilization, mitochondrial cytochrome c release, and caspase activation after porfimer sodium-PDT, which exacerbated cell death in human head and neck cancer (AMCNH3) cells [337]. In contrast, curcumin is also a potent antioxidant [336], a property that was found to reduce the extent of ROS production and thereby the degree of cell death in A431 human epidermoid carcinoma cells treated with rose bengal-PDT [338].

A recently discovered and rather specific inhibitor of HIF-1 activity is acriflavine (Table 1 ), which inhibits HIF-1 $\alpha$ /HIF$1 \beta$ and HIF- $2 \alpha /$ HIF- $1 \beta$ dimerization by binding the Per-ArntSim B domain of HIF- $1 \alpha$ and HIF-2 $\alpha$ [151]. The binding of acriflavine to HIF-1 blocks DNA binding and reduces transcriptional activity, tumorigenicity, and angiogenic signaling, as was demonstrated in xenotransplanted human prostate (PC3 ) and hepatoma (Hep3B) tumors in mice [151]. Results from our lab confirm the feasibility of employing acriflavine to improve PDT efficacy, as the sensitivity of A431 cells to PDT increased as a result of reduced glycolytic activity; downregulation of the HIF-1 target genes VEGF, PTGS2, and PAII; and upregulation of EDN1. Taken together, these results illustrate the importance of HIF-1 in the protection of tumor cells from PDT (Broekgaarden, M. et al., Nano Research, in resubmission; Weijer, R. et al., Oncotarget, in resubmission).

\subsubsection{Concluding remarks}

The HIF-1 transcription factor is essential for the survival of cells in a hypoxic environment and under conditions of acute stress, such as oxidative stress induced by PDT. The survival signaling manifests itself through direct intracellular events (e.g., by the production of prosurvival proteins and the shift to oxygen-independent metabolism) as well as through tissue processes (e.g., angiogenesis and proliferation) (Section 3.3.2). In case of PDT, it has been shown that HIF-1 activation causes the release of proangiogenic factors (Section 3.3.3).

Since malignant tumors proliferate faster than the rate of neovascularization, most tumors are hypoxic in nature and constitutively activate HIF-1 [316]. Poorly vascularized tumors may therefore be more resistant against PDT due to hypoxic preconditioning (in addition to the suboptimal accumulation of systemically administered photosensitizer molecules as a result of the poor blood supply). Tumors that overexpress HIF-1 are less sensitive to therapy and are associated with poor survival in patients. Accordingly, the coadministration of HIF1 inhibitors as neoadjuvants increases the efficacy of PDT, as has been demonstrated in several studies (Section 3.3.4).

\subsection{The ASK1 pathway}

The immediate early stress response is a mechanism in cells that encompasses the rapid transcription and translation of a set of genes coding for protein products that enable cells to adequately adapt to extra- or intracellular stress. Although the exact activation trigger fueling this response is somewhat elusive in relation to PDT, this section reviews the activation of ASK1 in response to generic oxidative stress, similar to that induced by PDT, and to TNF- $\alpha$ signaling (Section 3.4.1). ASK1 relays its signal via MAPKs (JNK and $\mathrm{p} 38^{\mathrm{MAPK}}$ ) to the AP-1 transcription factor family (Section 3.4.2.1.1 JNK and $\mathrm{p} 38$ proteins) that is responsible for the rapid induction of immediate early gene transcription. As a whole, the ASK1 signaling pathway exerts both cytoprotective as well as cytodestructive effects, depending on the balance between the activation of the ASK1 pathway and the NF-kB-TNF- $\alpha$ pathway that seem to chiefly govern cell fate (Section 3.2). The available literature on the participation of the ASK1 pathway in the post-PDT response (Section 3.4.5) and inhibition of MAPK activity (Section 3.4.4) are summarized, and possible inhibition strategies for this survival pathway are proposed.

\subsubsection{Activation mechanisms of ASK1}

ASK1 activation by ROS The activation of JNK, p38 ${ }^{\mathrm{MAPK}}$, and AP-1 transcription factors following oxidative stress is preceded by the activation of the mitogen-activated protein kinase kinase kinase ASK1 [339]. ASK1 forms homooligomers in its inactive state, comprising a complex that is referred to as the signalosome, in which multiple ASK1 proteins are bound at their C-terminal coiled-coil domains [340]. Thioredoxin (TRX) binds ASK1 subunits of the signalosome that shield the N-terminal transactivation domain, thereby inhibiting autophosphorylation of threonine (Thr) 845 that is required for signalosome activation [341]. Under oxidative stress (e.g., after TNF- $\alpha$-induced ROS formation), ROS (and oxidized substrates such as proteins and GSSG) mediate the oxidation of TRX [342]. TRX is oxidized at cysteine residues in the active site, leading to its dissociation from the signalosome, subsequent autophosphorylation of ASK1 subunits, and activation of the complex [339, 341, 343] (Fig. 6). Activated ASK1 phosphorylates MAP kinase kinases (MKK3), MKK4, MKK6, and MKK7 at conserved residues within the kinase domain, leading to their activation [344, 345]. MKK4 and MKK7 phosphorylate and activate JNK at Thr183 and Thr185, whereas MKK3 and MKK6 phosphorylate and activate the different $\mathrm{p} 38^{\mathrm{MAPK}}$ isoforms (Section 3.4.2.1 Acute downstreameffects of ASK1 activation) at Thr180 and Tyr182 [346, 347]. In addition to direct activation via oxidized TRX, ASK1 signaling may be enhanced via paracrine signaling through $\mathrm{TNF}-\alpha$, as is described in the following section. 


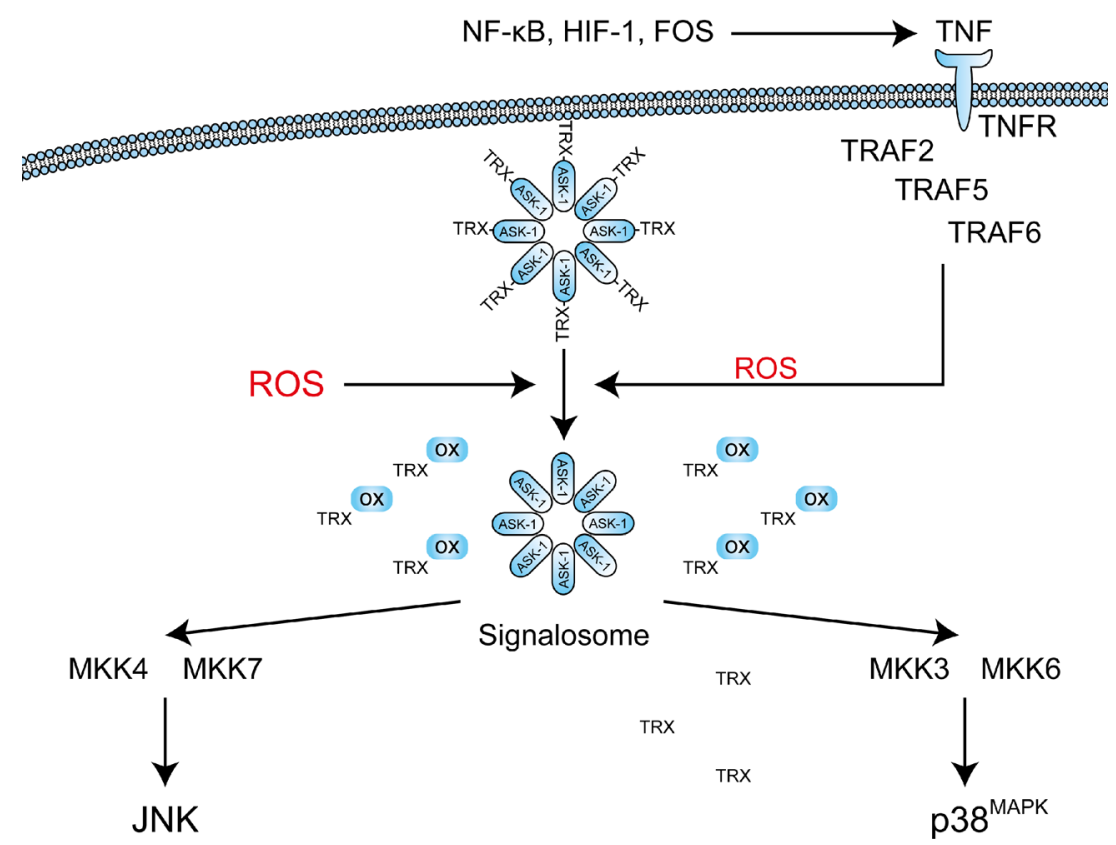

Fig. 6 Activation mechanisms of the ASK1 signaling pathway leading to JNK and $\mathrm{p} 38^{\mathrm{MAPK}}$ phosphorylation. ROS can directly or indirectly (via GSH) oxidize the TRX subunits (TRX-ox) of the inactivated signalosome complex. Upon oxidation and subsequent dissociation of TRX, the ASK1 heteromer autophosphorylates and initiates downstream signaling. MKK4/7 are kinases responsible for the activation of JNK proteins, whereas MKK3/6 phosphorylate and activate the $\mathrm{p} 38^{\mathrm{MAPK}}$.

ASK1 activation by TNF- $\alpha$ The ASK1 pathway is also stimulated by TNF- $\alpha$ (Fig. 6), which is expressed as a result of PDTactivated AP- 1 and NF- $\mathrm{kB}$ transcription factors, and TNF- $\alpha$ is a potent inducer of apoptosis and programmed necrosis (reviewed in [184]). TNF- $\alpha$ binds to the TNFR, which in turn mobilizes TRAFs 2, 5, and 6 to bind and activate the ASK1 signalosome [340]. ASK1 activation can also be triggered by TNF- $\alpha$ signaling alone [340] as a result of TNF- $\alpha$-mediated production of ROS by mitochondria and/or NADPH oxidase 1 [348, 349]. ROS production by TNF- $\alpha$ likely involves TRAF2 [350].

\subsubsection{Downstream effects of ASK1 activation}

Acute downstream effects of ASK1 activation Although the exact role of ASK1 itself is underinvestigated in the context of PDT, the ASK1 signaling cascade has been implicated in both increasing cell death and increasing cell survival in response to ROS or TNF- $\alpha$. Whereas a transient activation stimulus (i.e., short-lasting or mild oxidative stress) encourages survival and proliferation [351], a prolonged activating stimulus (i.e., severe and prolonged oxidative stress or prolonged TNF- $\alpha$ /TNFR signaling) promotes growth arrest and apoptosis [352] (Fig. 8). Since the MAPKs JNK and p38 ${ }^{\mathrm{MAPK}}$ can regulate both survival (via a plethora of AP-1-like transcription factors) and cause cell death (via the activation of proapoptotic BCL2 proteins), we postulate that these kinases
Downstream of the NF-kB, HIF-1, and FOS pathways, tumor cells and immune cells produce TNF- $\alpha$. TNF- $\alpha$ binds the TNFR, which activates intracellular TRAF. These TRAFs stimulate the production of mitochondrial ROS and NADPH oxidase 1-derived ROS that stimulate the dissociation of TRX from the signalosome, but also bind TRX to prevent reassociation of TRX with ASK1

are critical for this cell survival/death switch or checkpoint, as is discussed below and summarized in Fig. 7.

JNK and p38 proteins There are three JNK isozymes, namely JNK1, JNK2, and JNK3, whereby JNK3 is mainly expressed in neural tissue. JNK mRNA is prone to alternative splicing, giving rise to over 10 different isoforms, which explains the diverse biological functions in various tissues [353, 354]. Whereas JNK1 was found to be the most important inducer of downstream signaling and JNK2 is an antagonist that competes with JNK1 for ligand binding [355], a more activating role of JNK2 in downstream signaling induction has also been described [356]. Since most inhibition strategies for JNK signaling were performed with JNK1 inhibitors, the focus will be on this specific isozyme of the JNK family. Although there are four isoforms of $\mathrm{p} 38^{\mathrm{MAPK}}$ proteins, namely $\mathrm{p} 38 \alpha, \mathrm{p} 38 \beta, \mathrm{p} 38 \delta$, and $\mathrm{p} 38 \gamma$, the functions of $\mathrm{p} 38 \alpha$ and p38 $\beta$ are most extensively described [354]. Many inhibition strategies regarding $\mathrm{p} 38^{\mathrm{MAPK}}$ and downstream signaling events were performed with inhibitors for $\mathrm{p} 38 \alpha$ and $\mathrm{p} 38 \beta$, so the main focus for the remainder of this review will be on these specific isozymes of the $\mathrm{p} 38^{\mathrm{MAPK}}$ family.

AP-1 transcription factors JNK 1 and $\mathrm{p} 38 \alpha / \beta$ activate a multitude of AP-1-type transcription factors. The AP-1 transcription factor family consists of a large variety of dimers composed of basic leucine zipper domain (bZIP) proteins from the 


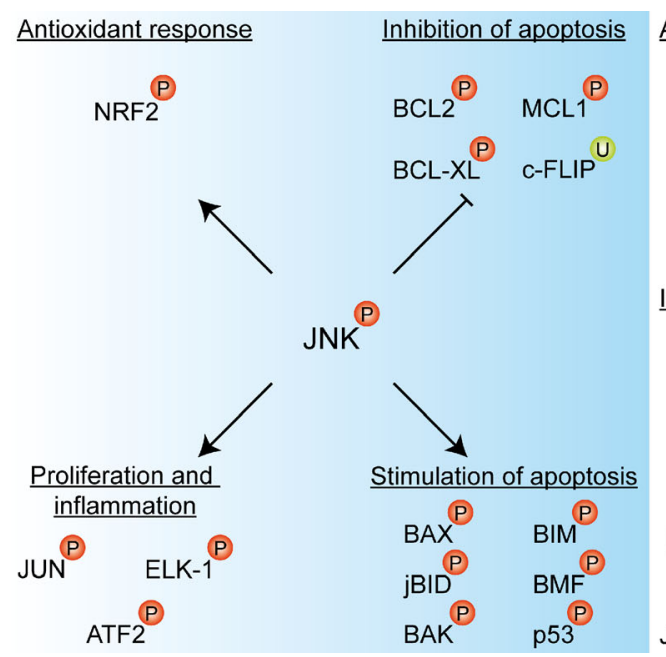

Fig. 7 JNK1 exerts kinase activity on several transcription factors and BCL2 family proteins through phosphorylation $(P)$ or ubiquitination $(U)$. Proliferation and inflammation are induced by phosphorylating members of the JUN and ATF2 protein family, as well as ELK-1. The NRF2 antioxidant response is triggered via phosphorylation of NRF2 at Ser40. Apoptosis is stimulated via the phosphorylation of BAX, BAK, BIM, $\mathrm{BID}$, and BMF proteins as well as via p53 activation. Antiapoptotic proteins are also phosphorylated (and inactivated) by JNK1, which include BCL2, BCL-XL, and MCL-1, or ubiquitinated (c-FLIP). The

JUN subfamily (JUN, JUNB, and JUND), the ATF2 subfamily (ATF2 and cAMP response element binding, CREB), and the FOS subfamily (FOS, FOSB, FOS-related antigen 1 (FRA1), and FRA2). Another subfamily exists, the MAF subfamily [262], but the function of these proteins in the cellular response to PDT or oxidative stress remains largely unexplored.

As addressed above, JNK1 specifically phosphorylates and activates AP-1 proteins from the JUN and ATF2 subfamilies as well as the non-AP-1 transcription factor ETS domaincontaining protein (ELK-1). Other transcription factors such as NRF2 and p53 are phosphorylated and activated by JNK1. p38 $\alpha / \beta$ also phosphorylates JUN and ATF2 proteins and also functions as a kinase for FOS proteins. Moreover, $\mathrm{p} 38 \alpha / \beta$ phosphorylates NRF2 [85] although the inhibitory effects of $\mathrm{p} 38 \alpha / \beta$ on NRF2 have been reported [357]. Phosphorylation of myocyte-specific enhancer factor 2 (MEF2) and CCAATenhancer binding protein (C/EBP) $\beta$ [354] has also been reported, thereby triggering the expression of a plethora of genes involved in cell type-specific differentiation. However, AP-1-type transcription factors constitute the main targets for these MAPKs, and a more elaborate description of the target genes of these transcription factors is provided below.

Proliferation JUN and ATF2 stimulate cell proliferation by upregulating the production of cyclins $\mathrm{D}, \mathrm{E}$, and $\mathrm{A}$; EGFR; heparin-binding EGF (HBEGF); and keratinocyte growth factor (KGF) [358-361]. Additionally, cell cycle inhibitors $\mathrm{p} 53, \mathrm{p} 19^{\mathrm{ARF}}$, and $\mathrm{p} 21^{\mathrm{CIP} 1}$ are downregulated by

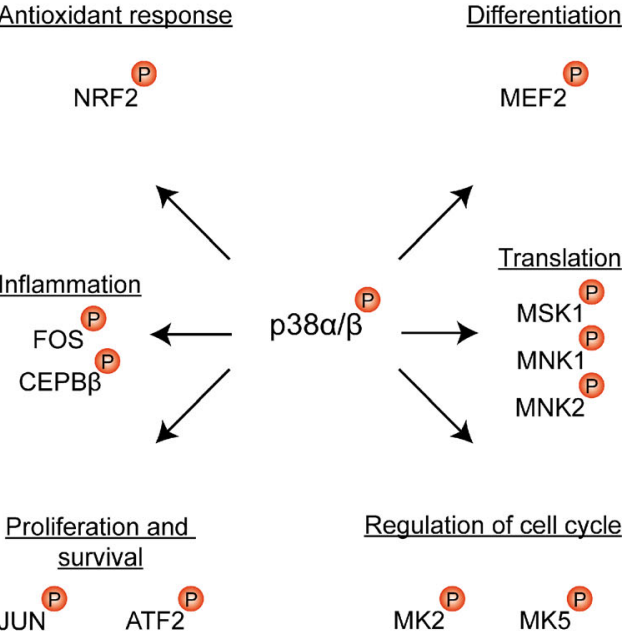

kinase functions of $\mathrm{p} 38 \alpha / \beta$ entail a similar effect on the NRF2 antioxidant response pathway as reported for JNK1. Inflammation is stimulated via phosphorylation of the AP-1 transcription factors of the FOS and C/EBP protein family. Proliferation and survival are promoted via activation of JUN and ATF2. Translation of newly transcribed genes is facilitated by phosphorylation of MSK1, MNK1, and MNK2. Differentiation is mediated via MEF2 activation by $\mathrm{p} 38 \alpha / \beta$. Additionally, p38 $\alpha / \beta$ regulate the cell cycle by phosphorylation of MK2 and MK5

JUN [362-365]. Contrastingly, JUN and ATF2 also upregulate several cell cycle inhibitors such as the retinoblastoma 1 protein (RB1), GADD $45 \alpha$ and $\beta$, and p16 ${ }^{\mathrm{INK} 4 \mathrm{~A}}[366,367]$.

Angiogenesis and invasion Stimulation of angiogenesis and invasion are the result of proinflammatory cytokines, growth factors, and extracellular matrix modifiers that ultimately stimulate tumor regrowth. ATF2 and FOS appear to play major roles in the production of proteins involved in these pathways. Moreover, $c-F O S$ is subject to upregulation by CREB, a member of the ATF2-like subfamily, which amplifies the FOS pathway [368]. FOS stimulates inflammation by facilitating the production of VEGF, urokinase-type plasminogen activator (UPA), uPA receptor (UPAR), MMP1, MMP3, methionyltRNA synthetase 1 (MTS1), Kelch-related protein 1 (KRP1), ferritin repressor protein (FRP), ezrin (EZR), and tropomyosin (TPM) 3 and 5b (reviewed in [262]). ATF2 further contributes to the generation of a proinflammatory state by mediating the production of platelet derived growth factor receptor A (PDGFRA) [369], MMP2 [370], TNF- $\alpha$ [371], IFN- $\gamma$ [372], and HSP90A5 [373]. In addition, CREB also induces many cytokines such as IL-2, IL-6, IL-10, and TNF- $\alpha$ to cause inflammation that in turn stimulates angiogenesis and invasion [374]. Besides directly stimulating apoptosis, many of the abovementioned cytokines are involved in stimulating immune cells to release a multitude of angiogenic factors via NF- $\mathrm{KB}$ (Section 3.2) and AP-1 transcription factors (Section 3.4). 
Apoptosis In addition to stimulating inflammation and proliferation, AP-1 transcription factors also regulate apoptosis following an oxidative insult. JUN regulates the transcription of antiapoptotic BCL2 family members BCL2, BCL3, BCL-XL, and the proapoptotic BIM [262], the eventual result depending on the extent of damage and the cross-talk between various pathways. Additionally, both JUN and FOS stimulate the extracellular apoptosis pathway by upregulating FAS ligand and FAS receptor (FASR) [262, 375], whereas ATF2 induces the production of TNF-related apoptosis-inducing ligand (TRAIL) [371]. Given the variety of different genes and processes influenced by the AP-1 transcription factor family and the overlap of genes that different family members can induce, the exact effects of AP-1 on overall tumor cell survival or cell death induced by PDT remain difficult to predict. This is because although AP-1 may stimulate tumor growth and survival by mediating cell cycle progression, inflammation, angiogenesis, and migration, AP-1 may also be instrumental in the induction of apoptosis via the upregulation of FAS, FASL, and TRAIL, as well the differential regulation of BCL2 protein family members.

Additional effects of p38 ${ }^{\text {MAPK }}$ To assist in transcription, p38 ${ }^{\mathrm{MAPK}}$ activates mitogen- and stress-activated protein kinases (MSK) 1 and 2 that phosphorylate histone $\mathrm{H} 3$ to enhance chromatin remodeling and transcription factor binding to DNA [376]. The activation of MAPK interacting kinases (MNK) 1 and 2 by $338^{\mathrm{MAPK}}$ further facilitates mRNA translation by phosphorylating the eukaryotic translation initiation factor (EIF)4E that binds RNA and targets it to ribosomes [377], whereas MSK1 contributes to mRNA translation by inactivating the EIF4E inhibitor 4E-binding protein 1 (4EBP1) [378]. Other functions of MSK1/2 include the phosphorylation and activation of transcription factors ATF1, CREB [379], as well as several other transcription factors (e.g., NF-kB, ETS variant 1, and high mobility group nucleosome binding domain 1). Through these transcription factors, MSKs upregulate the transcription of $J U N$ and FOS [379] and contribute to inflammation and survival by upregulating $I L-6$ and RELA (see NF-kB, Section 3.2) [376].

$\mathrm{p} 38 \alpha / \beta$ activity appears to stimulate cell motility by phosphorylation of MAPK-activated protein kinases 2 and 5 (MK2, MK5) [380]. When activated by $\mathrm{p} 38^{\mathrm{MAPK}}$, these kinases phosphorylate HSP27, causing HSP27 dimerization and consequent binding to the actin cytoskeleton - a phenomenon associated with heightened cell motility in human umbilical vein endothelial cells [381]. Thus, this activity of $\mathrm{p} 38 \alpha / \beta$ may stimulate tumor cell survival by promoting angiogenesis, invasion, and metastasis.

p38 $\alpha / \beta$ can have positive and negative effects on the cell cycle through the activation of MK2 and 5. MK2 halts the cell cycle by phosphorylating and activating the cell division cycle (CDC) proteins $\mathrm{CDC} 25 \mathrm{~B}$ and $\mathrm{CDC} 25 \mathrm{C}$, which can functionalize the G2/M checkpoint and arrest the cell cycle in the presence of
DNA damage. MK5 promotes senescence by phosphorylating p53 and inhibiting the expression of c-MYC, but also stimulates proliferation by sequestering ERK3 in the cytoplasm [382]. MK2 negatively regulates $\mathrm{p} 53$ by phosphorylating the $\mathrm{p} 53$ ubiquitin ligase MDM2 (mouse double minute 2, human homologue) and inhibits CDCs to stimulate proliferation despite DNA damage (reviewed in [383]). Other, but less studied effects of $\mathrm{p} 38^{\mathrm{MAPK}}$ include the upregulation of HIF-1 $\alpha$ [384] and COX-2 [385] (Sections 3.2 and 3.3), suggesting a survival-promoting role for $\mathrm{p} 38^{\mathrm{MAPK}}$. This has been corroborated in a recent study by Rubio et al., in which $\mathrm{p} 38^{\mathrm{MAPK}}$ was implicated in the removal of ubiquitin aggregates via autophagy and activation of NRF2 after hypericin-PDT that led to increased survival of fibroblasts [386].

Prolonged downstream effects of ASK1 activation Prolonged activation of JNK stimulates apoptosis. Prolonged JNK1 activation is a signal for extensive cell damage that triggers apoptosis via TNF- $\alpha$ and degradation of the caspase 8 inhibitor CFLAR [387, 388]. Apoptosis is further promoted through the inhibition of antiapoptotic BCL2 protein family members BCL2, BCL-XL, and MCL-1 [389, 390] in combination with activation of proapoptotic BAX, BAK, BIM, BCL2-modifying factor (BMF), and BID (yielding JNKcleaved BID or jBID) [391-393]. In addition, JNK1 stabilizes the tumor suppressor protein $\mathrm{p} 53$ to stimulate apoptosis and cell cycle arrest in response to DNA damage [345, 394]. Prolonged activation of JNK1 and consequent cell death signaling is induced by prolonged oxidative stress, depleted antioxidants and impaired survival responses (e.g., reduced activity of NRF2 and NF-kB), or TNF- $\alpha$ signaling combined with oxidative stress (Fig. 8). Similarly, in response to phorbol 12-myristate 13-acetate and ionomycin, transient activation of JNK1 was associated with survival of human Jurkat T-cells, whereas prolonged activation of JNK1 (phorbol 12-myristate 13-acetate, ionomycin, and UV-C irradiation) induced cell death [395]. In primary rat mesangial cells, TNF- $\alpha$ treatment alone induced transient JNK1 activation that did not result in loss of cell viability. Conversely, a combined treatment of TNF- $\alpha$ with either actinomycin D or cycloheximide resulted in prolonged JNK1 activation and major decreases in cell viability [396]. With the use of mouse embryonic fibroblasts (MEFs) derived from Traf $2^{-/-}$and $\mathrm{Traf6}^{-/-}$mice, Noguchi et al. revealed that Traf2 and Traf6 (typically activated via TNFR) were essential for the induction of $\mathrm{H}_{2} \mathrm{O}_{2}$-induced cell death [340], thereby indicating that simultaneous exposure of cells to TNF- $\alpha$ TNFR signaling and oxidative stress may facilitate prolonged ASK1 signaling with sustained activation of JNK1. ROS were an essential second messenger for TNF- $\alpha$ induced apoptosis in murine L929 cells, as the induction of apoptosis in murine $\mathrm{L} 929$ cells following combined $\mathrm{H}_{2} \mathrm{O}_{2}$ and TNF- $\alpha$ treatment could be completely prevented by the antioxidant N-acetylcysteine [341]. TNF- $\alpha$ inhibited ASK1/TRX interaction [341] — most likely via binding 
Acute activation mechanism

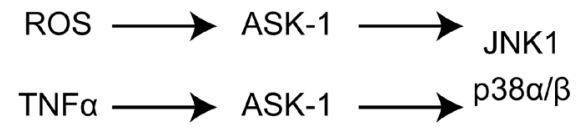

Transient repression mechanism

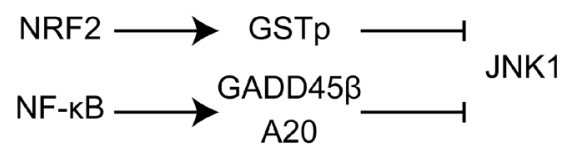

Prolonged activation mechanism

ROS +

TNFa

ASK-1

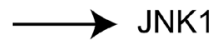

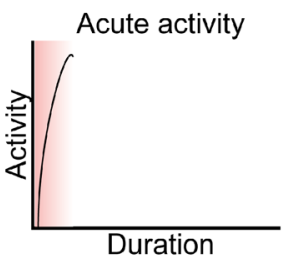

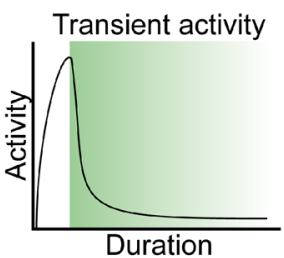

Duration

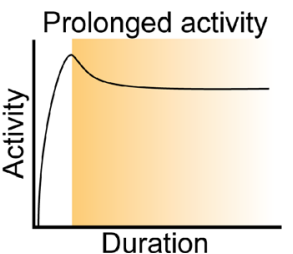

Survival

Survival

Apoptosis
Fig. 8 The ambivalent effects of the ASK1 pathway are dictated by the cross-talk between various pathways and the prevailing biochemical conditions. The primary activation mechanism of the ASK1 pathway by PDT emanates from oxidative stress or TNF- $\alpha$ signaling, leading to the acute, survival-promoting activity of JNK1 and p38 $\alpha / \beta$. Subsequently, downstream of the NRF2 and the NF- $\mathrm{kB}$ pathways, negative regulators

of TRAFs [340] - which could be reversed upon the addition of $\mathrm{N}$-acetylcysteine, suggesting an essential role for oxidants in the induction of TNF- $\alpha$-mediated apoptosis [341]. Downstream of TNF- $\alpha$, TRAF2 and RIP1 induced apoptosis in MEFs via JNK1 activation, although the involvement of ASK1 itself was not investigated [397]. The induction of cell death via TNF- $\alpha$ was prevented when NF- $\mathrm{KB}$ was activated in MEFs [350]. NF- $k B$ decreased TNF- $\alpha$-induced ROS formation, thereby preventing prolonged JNK1 activity and consequent cell death in MEFs [189]. A similar effect was found using PDT, in which inhibition of NF-kB with Bay 11-7082 in human glioblastoma cells augmented TNF- $\alpha$-induced tumor cell death following PDT [273]. However, the induction of necrosis by ALAPDT of human glioblastoma cells occurred via RIP1 and RIP3 in an NF-KB-independent manner [64].

Induction of transient JNK1 activity prevents apoptosis Several downstream genes regulated by NRF2 and $\mathrm{NF}-\mathrm{KB}$ in turn affect the transient activity of JNK1 by inhibiting its function to facilitate cell survival (Fig. 8). Since these gene products need to be transcribed and translated before being able to downmodulate $\mathrm{JNK} 1$, there is a time period during which JNK1 is active. GSTp is produced downstream of the NRF2 pathway and inhibits JNK1 to stimulate cell survival [398-402] or else transiently inactivates $\mathrm{p} 38^{\mathrm{MAPK}}$ to mediate survival of oxidatively stressed murine $3 \mathrm{~T} 3$ fibroblasts [402]. Moreover, the NF-KB and AP-1 target genes GADD $45 \alpha$ and $\beta$ limit the activity of JNK1 by binding and of JNK1 and $\mathrm{p} 38 \alpha / \beta$ are produced/activated that modulate the transient activation pattern of these kinases and thus promote cell survival. Whenever ROS and TNF- $\alpha$ signaling occur simultaneously, or whenever these stress signals endure, prolonged JNK1 and $\mathrm{p} 38 \alpha / \beta$ activation promotes apoptosis

inhibiting MKK 7 and 4 [403, 404]. Conversely, GADD45 $\alpha$ and $\beta$ increase the activity of $\mathrm{p} 38^{\mathrm{MAPK}}$, whereby the combined effects of JNK1 inhibition and p38 activation protected hematopoietic cells from UV-induced apoptosis [403]. Upregulation of A20 and XIAP by NF-kB leads to blocked JNK1 activity via an unknown mechanism (reviewed in [405]).

\subsubsection{Role of the ASK1 pathway in PDT}

Direct ASK1 activation following PDT has never been demonstrated, so the involvement of this pathway in response to PDT can only be deduced from the effects on downstream kinases and transcription factors. Considerable increases in $c-F O S$ and $c-J U N$ mRNA levels were found after porfimer sodium-PDT of RIF-1 cells. Levels of mRNA peaked 90 min post-PDT, after which mRNA levels gradually dropped to baseline during the subsequent $8 \mathrm{~h}$ [406]. Furthermore, protein kinase inhibitors such as staurosporine effectively blocked the synthesis of c-FOS mRNA, hinting toward the involvement of upstream kinases p $38^{\mathrm{MAPK}}$ and JNK [406]. Activation of JNK and $\mathrm{p} 38^{\mathrm{MAPK}}$, but not other MAPKs such as ERK1 or ERK2, in benzoporphyrin derivativePDT-subjected murine PAM212 keratinocytes confirmed the involvement of the AP-1 response. The activation of JNK and $\mathrm{p} 38^{\mathrm{MAPK}}$ was abrogated in the presence of antioxidants [407]. Activation of the AP-1 response in the PAM212 keratinocytes was further confirmed by ATF2 and JUN phosphorylation following PDT [407]. 


\subsubsection{Inhibition strategies of ASK1 and its downstream targets}

Since the discovery of their involvement in the response to PDT, the effects of the MAPKs and AP-1 proteins on the biological fate of PDT-affected cancer cells have been elusive or inconsistent. Two types of approaches can be used to inhibit the ASK1 pathway. The first approach is to inhibit the function of AP-1 transcription factors, while the other method prevents AP-1 activation through inhibition of the upstream kinases JNK and $\mathrm{p} 38^{\mathrm{MAPK}}$. Direct chemical inhibition of AP1 transcription factors is possible with retinoic acid and its analog SR11302 (Table 1). More specifically, retinoic acid or retinoic acid analogs are able to inhibit ATF2 [408] and cJun [409]. It was shown that these inhibitors were efficient in blocking AP-1 activity, which prevented tetradecanoyl phorbol acetate-induced papilloma formation in mice [152, 410]. This illustrates the potential for a treatment modality in which retinoic acid is used to sensitize tumor cells to PDT. However, to date no studies have employed direct inhibition of AP-1 in conjunction with PDT.

Indirect inhibition of AP-1 activation has been achieved with inhibitors of JNK and $\mathrm{p} 38 \alpha / \beta$. JNK1 can be inhibited by the anthrapyrazole SP600125 (Table 1), even though this compound also mildly impairs the function of $\mathrm{p} 38 \beta$ and ERK2 [153]. The pyridinyl imidazole derivatives SB202190, SB203580, and PD169316 have been used for the selective inhibition of $\mathrm{p} 38 \alpha / \beta$, but not the $\delta$ or $\gamma$ isozymes (Table 1$)$. The JNK and $338^{\mathrm{MAPK}}$ inhibitors all compete with ATP for the ATP-binding domain of the kinases, resulting in the reversible inhibition of kinase activity [154]. However, the roles of these kinases are rather unpredictable and the effects of inactivation are dependent on the cell type and the extent of damage. The contradictory effects that these MAPKs can have on tumor cell survival following PDT are shown in Table 2. The data reveal that $\mathrm{p} 38^{\mathrm{MAPK}}$ and JNK induce both survival and apoptosis but also suggest that the inhibition of $\mathrm{p} 38 \alpha / \beta$ augments tumor cell death, whereas inhibition of JNK either has no effect or impairs PDTinduced cell death. This is in line with the dichotomous effects of JNK1 activation and the cytoprotective function of $\mathrm{p} 38 \alpha / \beta$ as described in Section 3.4.2.2 Prolonged downstream effects of ASK1 activation, in which p38 $\alpha / \beta$ mainly functions as activator of AP- 1 and facilitators of transcription. Therefore, it can be deduced that JUN and ATF2 (both downstream of JNK) do not play a significant role in cell survival, but FOS (only activated by $\mathrm{p} 38^{\mathrm{MAPK}}$ ) may in fact be a decisive factor for cell survival. Alternatively, in a recent study by Rubio et al., $\mathrm{p} 38^{\mathrm{MAPK}}$ was implicated in the removal of ubiquitin aggregates via autophagy and activation of NRF2 after hypericin-PDT, which resulted in increased survival of fibroblasts [386].

\subsubsection{Concluding remarks}

The ASK1 pathway is one of the most complicated pathways activated by PDT. Although ASK1 itself has, to our knowledge, never been investigated in the context of PDT, many of its downstream targets have often been implicated in cellular responses to PDT. However, the effects of this pathway are highly divergent, ranging from stimulation of survival to causing inflammation and stimulation of cell death. It is arguable that JNK1 has a particularly important role in the stimulation of apoptosis in cells exposed to severe and prolonged oxidative stress or concomitant TNF- $\alpha$ signaling. Experimental evidence regarding the inhibition of JNK-1 and $\mathrm{p} 38 \alpha / \beta$ is in agreement with this hypothesis. Survival signaling as a result of transient JNK and $\mathrm{p} 38^{\mathrm{MAPK}}$ activity may arise from the phosphorylation of AP-1 transcription factors and the triggering of the immediate early survival response. Therefore, given the dichotomous nature of the ASK1-MAPK pathway, it is hypothesized that pharmacological inhibition of AP-1 transcription factor activation could improve PDT efficacy, while the function of the MAPKs JNK and p $38^{\mathrm{MAPK}}$ should remain intact.

\subsection{The proteotoxic stress response}

ROS production by PDT primarily results in the oxidation of lipids and proteins, leading to protein misfolding or formation of protein aggregates [27]. These forms of proteotoxic stress trigger transcriptional responses that are collectively known as the UPR, which is considered a form of ER stress. The UPR is mediated by several transcription factors that include inositolrequiring protein 1 (IRE1), ATF6, and protein kinase RNA-like ER kinase (PERK). Although generally not included as a part of the UPR, unfolded proteins generated as a result of oxidative and proteotoxic stress also activate HSF transcription factors, which aid in alleviating ER stress [419]. The HSF transcription factors facilitate an adaptive response that enables protein refolding and degradation of protein aggregates via the upregulation of chaperones and inhibition of protein neogenesis (reviewed in [420]).

It can be seen that, when the adaptive responses to unfolded proteins and ER stress are constitutively active in tumor cells, the threshold for the ROS-mediated induction of cell death will be higher. HSFs trigger the production of HSPs to assist in protein refolding, protein complex formation, or protein degradation to alleviate the proteotoxic stress [421, 422]. Clinical evidence has shown that HSF1 and its downstream products HSP27, 70, and 90 are often constitutively activated in tumors [423]. The same constitutive activation was found for components of the IRE1 and PERK pathways [424], thus pointing toward a predisposition of tumor cells to be able to cope with PDT-induced proteotoxic stress. 


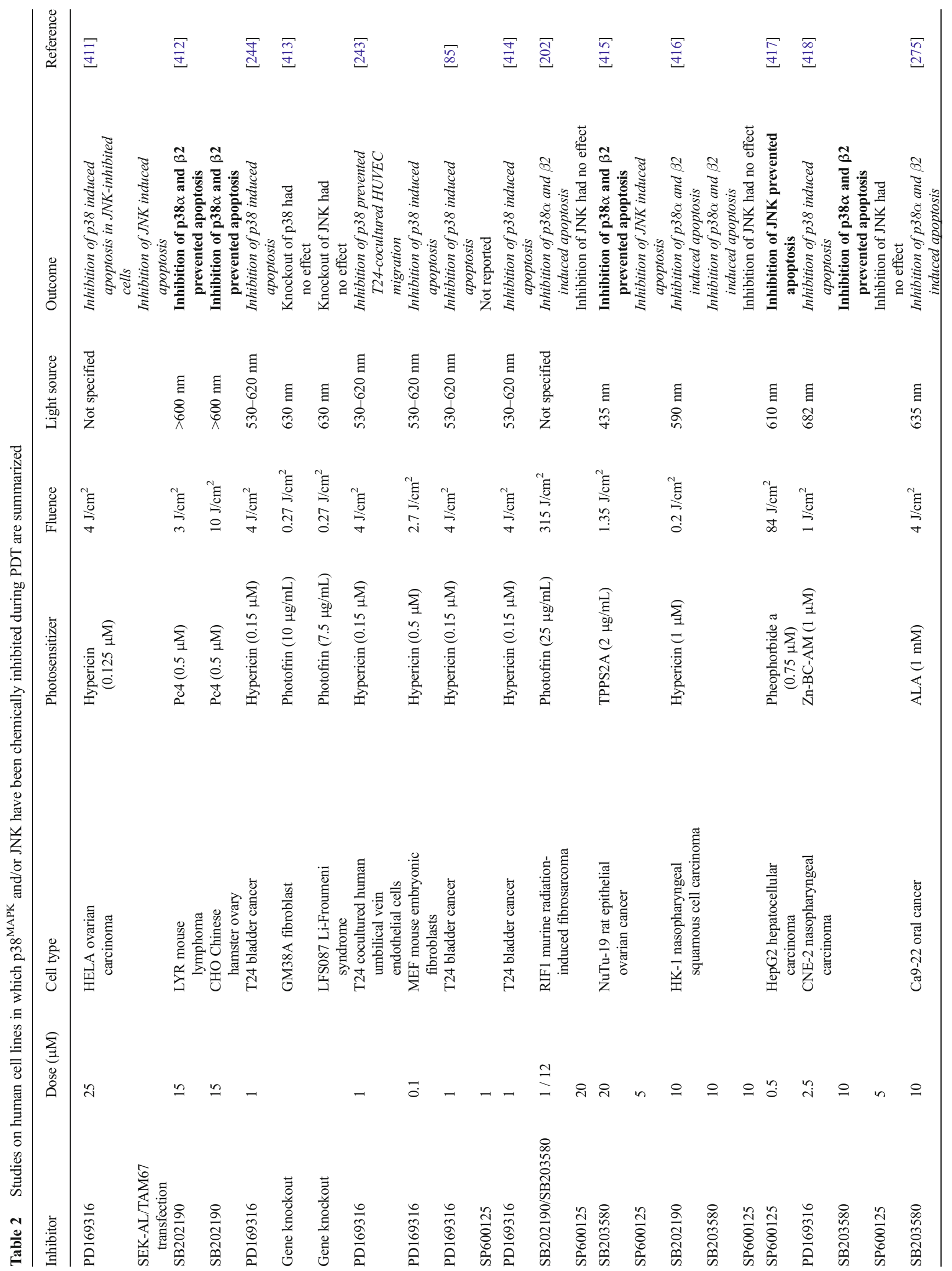




\subsubsection{Activation mechanisms of the proteotoxic stress response}

Of the four HSFs isoforms 1-4, HSF1 is the most important [421] and will be discussed in the context of the proteotoxic stress response. Under nonstressed conditions, HSF1 forms inactive monomers in the cytoplasm that are acetylated by p300/CREB binding protein (CBP). HSP40, 70, and 90 form complexes with HSF1 and negatively regulate either its transactivation mechanism (HSP90) or its DNA-binding capacity (HSP40 and HSP70) [421, 425]. Whenever HSPs need to be engaged to combat proteotoxic stress by ROS or hypoxia, HSPs dissociate from the HSF complex and bind to misfolded proteins. This binding relieves the negative regulation of HSF, leading to HSF homotrimerization and activation [421]. Moreover, stress-induced sirtuin (SIR2) deacetylates HSF and maintains the complex in a state capable of binding DNA [425] (Fig. 9). Additionally, JNK2 has been implicated in phosphorylating the transactivation domain of HSF1, contributing to its activation and subsequent binding to genomic

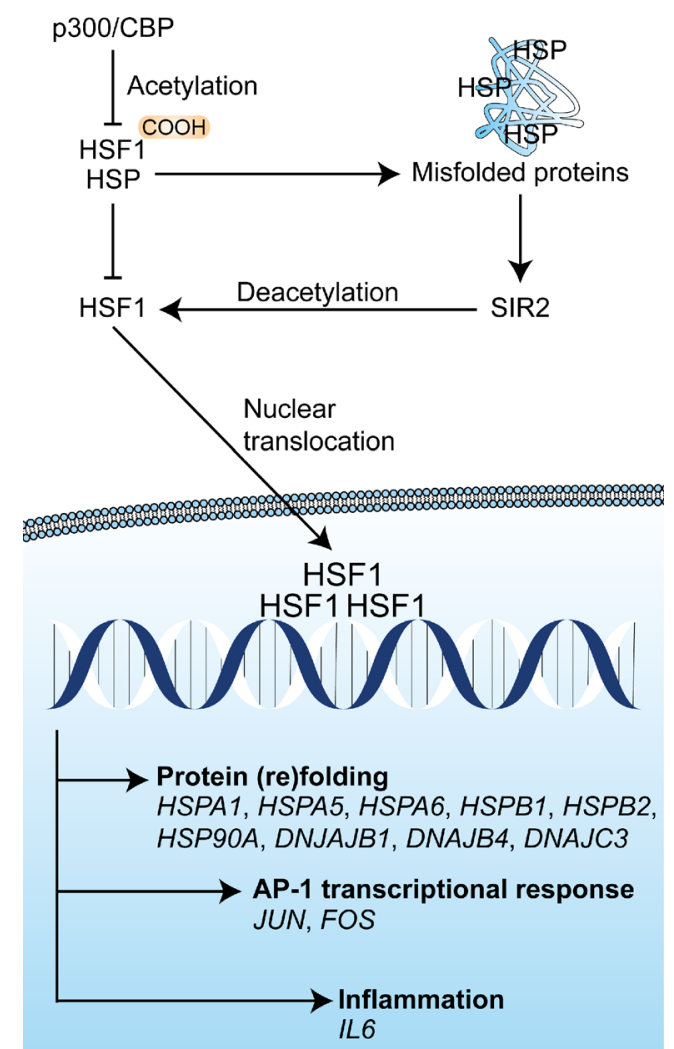

Fig. 9 Mechanism of HSF1 activation. Monomeric HSF1 is kept inactive in the cytosol via acetylation by $\mathrm{p} 300 / \mathrm{CREB}$ binding protein. The cytosolic monomers interact with HSP40, 70, or 90, which prevents DNA binding and transactivation. Proteotoxic stress titrates HSPs away from HSF1 and stress-induced SIR2 deacetylates the HSF1 monomers. Subsequently, HSF1 forms homotrimers, translocates to the nucleus, and binds genomic heat shock elements to facilitate target gene expression. The target gene products are mainly involved in protein (re)folding, but also enforce the immediate early stress response and inflammation 
heat shock elements [426]. Other sensors of the ER stress response include IRE1, ATF6, and PERK. These proteins are embedded in ER membranes and are activated when their chaperones (mainly HSP70A5, also known as glucoseregulated protein 78 (GRP78) and binding immunoglobulin protein (BIP)) are recruited away to bind to unfolded proteins (Fig. 10).

\subsubsection{Downstream effects of HSF1, IRE1, PERK, and ATF6}

HSF1 HSF1 induces the expression of a plethora of heat shock proteins (including but not limited to HSP27, HSP40 (DNAJ), HSP70, and HSP90 [427]), which are molecular chaperones that assist in the folding, translocation, and complexation of newly translated proteins (Fig. 9). Increased expression of HSPs enhances the survival of cells that have been stressed by prooxidative conditions, hypoxia, or chemotherapeutic agents [428]. Consequently, the constitutive activation of the heat shock response in tumors (as a result of constitutive mild stress) is frequently observed and protects tumor cells from the cytotoxicity involved in different kinds of cancer therapy (chemotherapy, radiotherapy, and PDT) [429, 430]. HSF 1 also induces $J U N$ and FOS (Fig. 9), thereby reinforcing the AP-1-induced immediate early stress response (Section 3.4.2.2 Prolonged downstream effects of ASK1 activation) [431, 432]. Other, less studied effects of HSF1 are the induction of $I L 6$ (Fig. 9) and the consequent transcriptional repression of TNFA, IL1B, NOS2, CCL5, IL8, C5, and ICAM1 by inhibiting NF-kB activation (reviewed in [433]). The HSF1 pathway most likely raises the level of intracellular stress necessary for IKK activation and IKB $\alpha$ degradation that are required to activate NF- $\mathrm{KB}$ [433]. The reduction of proteotoxic stress emanating from oxidatively modified proteins has been attributed to the chaperone function of HSPs, which tend to prevent protein denaturation and aggregation, assist in the correct refolding of affected proteins, promote GSH reduction, and maintain proteasome function (reviewed in [428, 429, 434]). Moreover, HSP70 prevents apoptosis by reducing JNK1 phosphorylation and BIDmediated mitochondrial membrane permeabilization; preventing caspase 8 activation by FADD; lessening the mitochondrial localization of BAX; inhibiting cleavage of procaspases 3, 7, and 9; and binding apoptosis-inducing factor (AIF) and a variety of other apoptotic effectors [429]. HSP27 is also an inhibitor of apoptosis by sequestering cytochrome $\mathrm{c}$ when released from the mitochondria, preventing the mitochondrial release of second mitochondria-derived activator of caspases (SMAC), blocking the activation of the proliferative PI3K-AKT pathway that enables cells to divert energy consumption toward relieving the ER stress, and stimulating prosurvival NF- $\mathrm{kB}$ signaling [430]. The latter effect is somewhat controversial given the consensus that downregulation of many NF- $\mathrm{KB}$ target genes can be attributed to the HSPdependent inhibition of NF-KB activation, suggesting a complicated relationship between the HSF pathway and the NF-kB pathway.

IRE1, ATF6, and PERK Besides HSF1, downstream events related to proteotoxic stress are also induced by the IRE1, ATF6, and PERK. IRE1 has kinase activity and RNAse activity via which it stimulates autophagy and apoptosis. The cytosolic domain of IRE1 complexes with TRAF2 to activate ASK1, resulting in prolonged, proapoptotic JNK1 activation. Autophagy is stimulated by IRE1 via the splicing of XBPI mRNA, resulting in the accumulation of an active XBP1 transcription factor. XBP1 upregulates the production of
Fig. 10 Activation mechanism of the ER stress response as a result of proteotoxic stress. Unfolded protein detectors IRE1, PERK, and ATF6 are sequestered by HSP70A5. Upon proteotoxic stress due to protein oxidation, misfolding, or formation of protein aggregates, HSP70A5 is recruited toward the misfolded proteins. The ER stress detectors are subsequently activated, leading to the initiation of XBP1, ATF4, and ATF6 transcription factor function

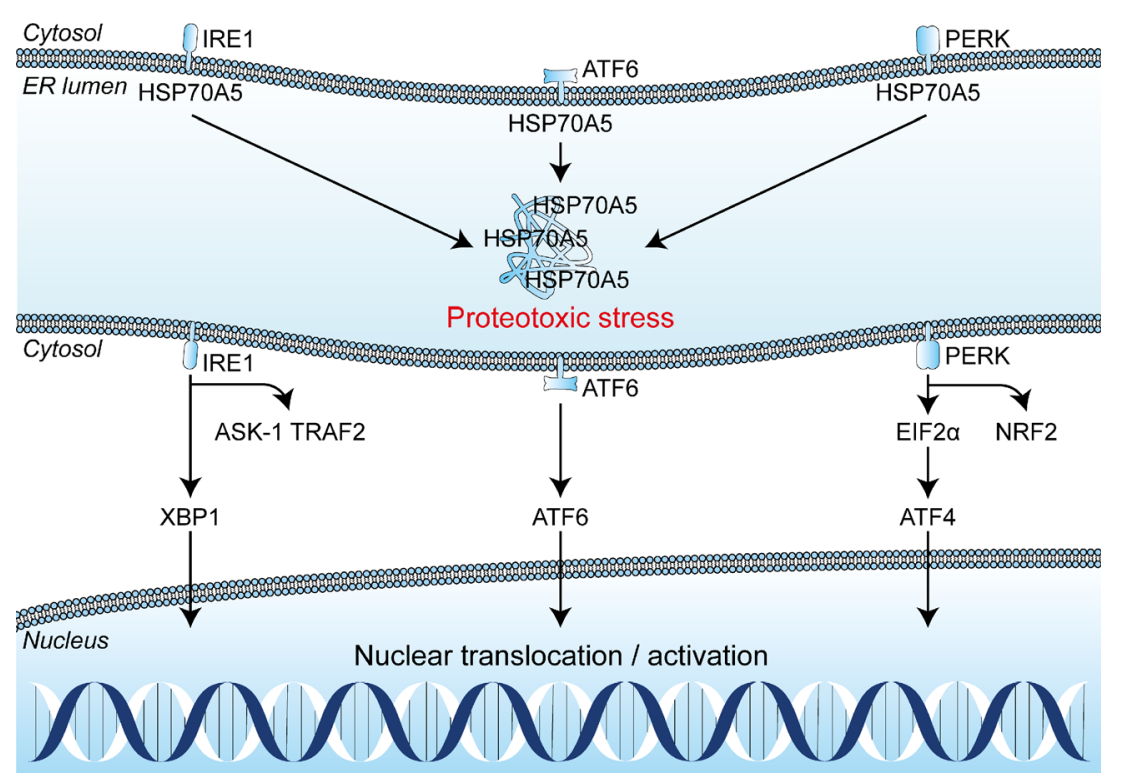


HSP70A5, protein disulfide-isomerase (PDI)P5, HSP40B9, ubiquitin-conjugating enzyme E2E1, and the ER degradation-enhancing $\alpha$-mannosidase-like protein 1 (EDEM1) [435] (Fig. 11) that all aid in refolding and degradation of misfolded proteins, a process termed ER-associated degradation (ERAD) [436]. ERAD is a form of autophagy through which terminally misfolded proteins and protein complexes are targeted for proteasomal degradation, eventually reducing proteotoxic (ER) stress [436]. Additional target genes of XBP1 include XBP1 and ATF6A as well as several other genes with a diverse range of functions [435] (Fig. 11).

ATF6 is also activated by proteotoxic stress and initiates the transcription of chaperones and ERAD-associated genes. These chaperone genes include HSPA5 (HSP70A5), $H S P 90 B 1$, and CRT (calreticulin, CRT). ATF6 additionally triggers the expression of ERAD-stimulating genes such as $X B P 1, P D I$, yeast Der1-like protein (DERL1), homocysteine-induced ER-protein $(H E R P)$, synovial apoptosis inhibitor 1 ( $S Y V N 1)$, and suppressor of Lin-12-like (SEL1L) [437]. ATF6 also upregulates C/EBP homologous protein (CHOP, encoded by DDIT3) to promote apoptosis [436, 438] (Fig. 11).

Activated PERK phosphorylates and activates NRF2 (Section 3.1) and EIF2 $\alpha$, resulting in activation of the antioxidant stress response and general inhibition of translation yet the selective translation of ATF4 mRNA. In turn, ATF4 stimulates both apoptosis and survival. It upregulates the expression of proapoptotic proteins such as CHOP, p53-upregulated modulator of apoptosis (PUMA, or BCL2-binding component 3 $(B B C 3)$ ), GADD34 (or protein phosphatase 1, regulatory subunit 15a (PPP1R15A), tribbles-related protein 3 (TRIB3), and BIM (BCL2L11) [437]. Survival is promoted via stimulation of amino acid metabolism, protein (re)folding, and restoration of redox homeostasis [439, 440] (Fig. 11). The latter function is achieved via HO-1 upregulation by complex formation with NRF2 [441]. Interestingly, ATF4 is activated by hypoxia and plays an important role in resistance to cancer therapy in a similar fashion to HIF-1 [440]. Interested readers are referred to more elaborate reviews on ER stress and the UPR [420, 425].

\subsubsection{Role of the proteotoxic stress response in PDT}

PDT was found to activate HSF $[442,443]$ and stimulate the production of HSP70, HSP47, HSP60, and HSP27 [442, 444-450]. Furthermore, high levels of HSP27, HSP60, HSP70, and HSP90 were linked to reduced susceptibility of tumor cells to PDT in vitro and in vivo [250, 444, 448, 450, 451]. The cytoprotective properties of HSPs after PDT likely arise from the alleviation of proteotoxic stress that ensues protein oxidation. The induction of ER stress by PDT was studied by Szokalska et al., who showed that porfimer sodium-PDT leads to extensive protein carbonylation, polyubiquitination, and widening of the ER lumen [27]. Moreover, PDT induced XBP1 activation and upregulation of HSP70A5 and calnexin, whereby ERAD was crucial for the survival of EMT-6 and HeLa cells after PDT in vitro and in vivo [27]. A microarray looking at the transcriptional response of T24 bladder cancer cells to hypericin-PDT showed significant mRNA upregulation of HSP70A5, 40, 47, 60, 90, 110, CHOP, GADD34, XBP1, PERK, ATF3, and ATF4, providing compelling evidence for the involvement of HSF1 and the UPR in response to PDT [124]. A follow-up study by the same group suggested that PERK-upregulated NOXA was the main instigator of tumor cell death after hypericin-PDTinduced ER stress [452].

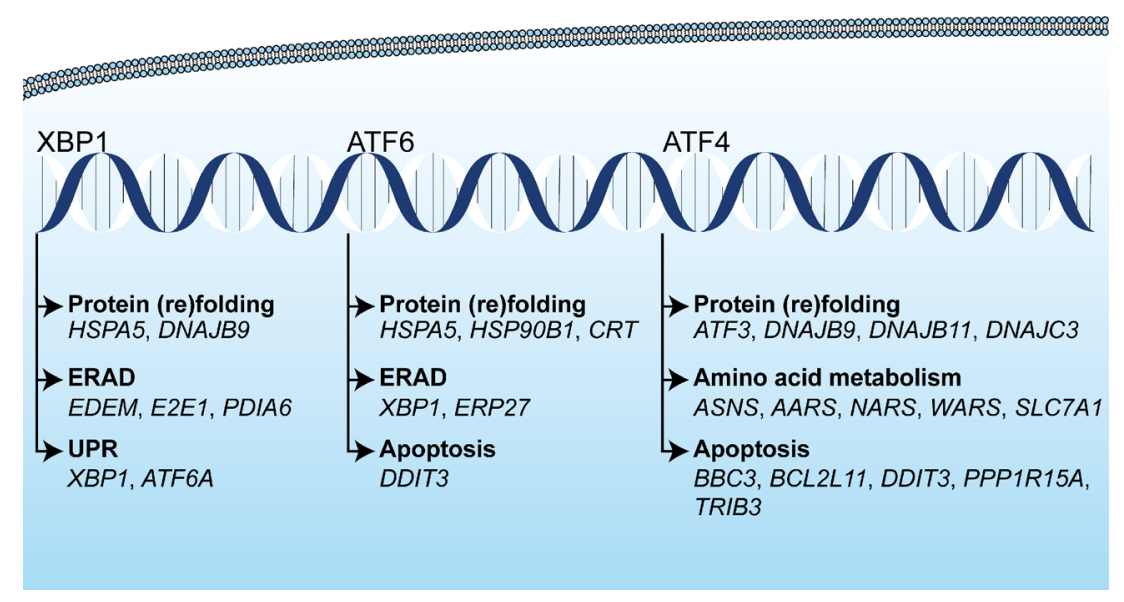

Fig. 11 Transcriptional regulation of genes induced by XBP1, ATF6, and ATF4 in response to proteotoxic stress. XBP1 stimulates protein (re)folding, ERAD, and amplifies the UPR. ATF6 also promotes protein (re)folding and ERAD, but also stimulates apoptosis by upregulating DDIT3 (CHOP). ATF4 ameliorates proteotoxic stress by upregulating $A T F 3$ and a plethora of DNAJ genes (encoding various isoforms of
HSP40). ATF4 additionally upregulates genes involved in amino acid metabolism that include, but are not limited to, asparagine synthetase $(A S N S)$, alanyl-tRNA synthetase (AARS), asparagyl-tRNA synthetase (NARS), tryptophanyl-tRNA synthetase (WARS), and the cationic amino acid transporter $S L C 7 A 1$. ATF4 additionally upregulates proapoptotic genes BBC3, BCL2L11, DDIT3, PPPIR15A, and TRIB3 
With respect to the antitumor immune response, HSP70 in particular has been implicated in immune cell modulation after PDT [199, 422]. Apoptotic cells expressed HSP70 on their plasma membrane after PDT [445], most likely in an attempt to stabilize the plasma membrane or chaperone integral membrane proteins [422]. Moreover, HSP70 can bind protein fragments derived from tumor-specific antigens such as mutated, truncated, or misfolded proteins. When expressed on the plasma membrane or released from necrotic cells, these HSP70/ tumor antigen complexes can be taken up by dendritic cells to induce their maturation, activation, and migration to lymph nodes, where they can initiate cross-presentation to naive Tcells and stimulate the formation of tumor-specific $\mathrm{CD}^{+}$cytotoxic T cells (reviewed in [199]). A similar mechanism in favor of dendritic cell activation has been ascribed to CRT (a downstream gene product of ATF6), which aids in ER protein folding. When CRT is expressed on the outer leaflet of the plasma membrane (ecto-CRT), it is associated with the induction of immunogenic cell death that stimulates antigen presentation and the activation of immune cells. Following hypericin-PDT (hypericin localizes to the ER), T24 cells expressed ecto-CRT in a PERK-dependent manner. Moreover, PERK was essential for the immunogenicity of CT26 cells treated with hypericin-PDT in vivo [453]. Thus, the proteotoxic stress response seems to play a key role in mediating an antitumor immune response.

\subsubsection{Inhibition strategies for the proteotoxic stress response and its downstream targets}

It may be postulated that inhibition of HSPs would be beneficial when beneficial for PDT outcome given the role of HSPs in tumor cell survival [422]. However, their role in promoting the antitumor immune response also suggests that HSP inhibition may be detrimental [199]. Ferrario and Gomer used the geldanamycin derivative 17-AAG [155] to inhibit the function of HSP90 (Table 1) during PDT and found a reduction in protein levels of survivin, VEGF, phospho-AKT, and BCL2. A higher cure rate and long-term survival were observed in BA mammary tumor-bearing mice treated with PDT combined with 17-AAG $[250,252]$. HSP70 inhibition with the bacterial cytotoxin SubA fused to EGF [160], (Table 1) was recently shown to augment the efficacy of porfimer sodiumPDT in human SW-900 lung cancer cells and DU-145 prostate cancer cells as a result of increased ER stress [454]. Taken together, these results point toward the beneficial effect of HSP inhibition in the enhancement of PDT efficacy. Besides 17-AAG, other HSP90 inhibitors are available and include different geldanamycin derivatives, although these may be associated with liver toxicity [455], as well as the synthetic small molecules CNF-2024/BIIB-021, NVP-AUY922, SNX5422, and STA-9090 (Table 1), which are undergoing clinical trials [156-159, 456]. However, inhibition of HSP90 typically exacerbates proteotoxic stress that induces HSP70 proteins [457] and may therefore alleviate any beneficial effects of these agents in terms of tumor cell death.

Alternatively or in addition to HSP90 inhibition, HSP70 inhibitors are also available. Schlecht et al. recently demonstrated the inhibition of HSP70 and HSC70 (a constantly expressed isozyme of HSP70) using VER-155008, a compound that binds the nucleotide binding domain of these proteins and reduces their ATPase activity (Table 1). In RNAi knockdown experiments, it was shown that concomitant inhibition of HSP70 and HSC70 was necessary to induce tumor cell death [161]. A more effective approach to completely abolish the heat shock response is to block HSF1 activity. KRIBB11 $\left(N^{2}-\left(1 \mathrm{H}\right.\right.$-indazole-5-yl)- $N^{6}$ methyl-3-nitropyridine-2,6-diamine) is an HSF1 inhibitor that blocks the association between HSF1 and positive elongation factor $b$, which is required for HSF1 transcriptional activity (Table 1). Accordingly, KRIBB11 was very effective in preventing HCT-116 tumor growth in nude mice [458]. Based on these results, inhibitors of the HSF pathway could be used to elucidate the role of this pathway in PDT and may provide promising approaches to improve PDT efficacy.

During ER stress, cells deal with the accumulation and aggregation of carbonylated proteins by polyubiquitination and proteasomal degradation. Therefore, Szokalska et al. investigated whether inhibition of the proteasome could exacerbate ER stress and increase the extent of cell death after PDT. Indeed, porfimer sodium-PDT on EMT-6 and HeLa cells pretreated with $4 \mathrm{ng} / \mathrm{mL}$ bortezomib (binds and inhibits the catalytic center of the 26S proteasome [162], Table 1) for $24 \mathrm{~h}$ increased the accumulation of carbonylated proteins and disrupted ERAD, leading to an increased sensitivity of cells to PDT [27]. Similar results were obtained for verteporfinPDT in combination with bortezomib $(2 \mathrm{mg} / \mathrm{kg})$ in a PC-3 mouse xenograft model [459]. Thus, these results attest to the utility of pharmacological interventions in proteasome function as a means to augment ER stress and improve the therapeutic efficacy of PDT. Pharmacological inhibition of IRE1 and ATF6 (but not PERK) is possible with 4phenylbutyric acid analogs (Table 1), although the exact mechanism has not been elucidated [163]. With respect to PERK, inhibition is possible with the synthetic compound GSK2656157 (Table 1), which competes with ATP to bind PERK specifically, and thus inhibits its kinase activity [164]. However, none of these UPR-inhibiting compounds have been investigated in combination with PDT.

\subsubsection{Concluding remarks}

Proteotoxic stress appears to be a primary response to PDT regardless of cell type and PDT strategy. The degree to which this response is triggered depends somewhat on the photosensitizer localization insofar as ER-localizing photosensitizers such as hypericin are more effective in inducing the UPR than 
photosensitizers that accumulate in other intracellular venues. While the functional outcome of this pathway may be both protective and destructive in tumor cells, the protective effects of the proteotoxic stress response can be pharmacologically blocked to promote tumor cell death. Inhibition of HSP70 and HSP90 was shown to increase the efficacy of PDT, as did inhibition of the proteasome by exacerbating ER stress. The HSF pathway is an essential component of the UPR in response after PDT. Given its reported induction by hypoxia and its constitutive activation in tumor cells [460], the UPR may protect tumors against anticancer therapies [424] such as PDT. Disrupting the cytoprotective effects of the UPR or interfering with the function of chaperones has been shown to enhance proteotoxic stress and stimulate cellular demise after PDT. Thus, the proteotoxic stress pathway is an important and feasible target for pharmacological interventions to enhance the therapeutic efficacy of PDT.

\section{Concluding remarks}

Tumor cells have the intrinsic ability to adapt to potentially harmful situations, such as those induced by chemotherapy, radiotherapy, and PDT. With respect to PDT, the activation of NRF2, NF-KB, HIF1, ASK1, HSF1, IRE1, PERK, and ATF6 and the effects of their downstream protein and gene targets have been reviewed. Together, these transcription factors and kinases facilitate the survival of tumor cells that suffer from a disrupted redox balance, low oxygen availability, apoptotic signaling, and oxidative damage to proteins.

The pathways that have the highest potential for pharmacological inhibition with the aim to improve the therapeutic efficacy of PDT are those from which no proapoptotic stimuli emerge. In that respect, blocking the NRF2, HIF1, and HSF1 pathways holds the highest potential to reduce the extent of tumor cell survival post-PDT. This is reflected by the substantial amount of evidence in which the inhibition of one or more of the downstream protein products (e.g., HO-1, COX-2, HSP70) from these pathways has led to increased efficacy of PDT. Unfortunately, the conclusion is not that straightforward regarding the ASK1 pathway. The ASK1 signaling axis mainly promotes survival via transient JNK1 and $\mathrm{p} 38^{\mathrm{MAPK}}$ activity and their induction of the AP-1 transcription factors. However, upon prolonged oxidative stress and corollary TNF- $\alpha$ signaling, JNK1 has potent proapoptotic activity. Thus, selective inhibition of $\mathrm{p} 38 \alpha / \beta$, but not the complete ASK1 signaling cascade, may be therapeutically beneficial for PDT, as is evidenced by the available literature on this topic (Table 1). The transcriptional events emanating from the activated UPR transcription factors IRE1, ATF6, and PERK are also challenging with respect to designing a pharmacological inhibition strategy. Whereas no proapoptotic signaling appears to arise from IRE1, both ATF6 and PERK promote apoptosis via the induction of, e.g., CHOP. Moreover, the multitude of potential target genes and effects make it arduous to predict the results of an inhibition strategy in conjunction with PDT. Thus, there is an explicit need for further investigations regarding the importance of these particular pathways in the cellular response to PDT. Inhibition of the NF-KB pathway appears unwise given its strong proinflammatory function and its potential to induce programmed cell death. It is probable that some downstream targets of this pathway are very strong inducers of tumor cell survival (i.e., COX-2 and survivin), yet completely abolishing this pathway has not produced convincing evidence that pharmacological inhibition is feasible in combination with PDT. Thus, the ambiguous downstream effects of the AP-1, UPR, and NF- $\mathrm{KB}$ pathways illustrate an obvious pitfall in applying a pharmacological inhibition strategy for these signaling cascades, since blocking a particular pathway also diminishes any proapoptotic effects of that pathway. A less obvious risk is the use of a compound that is capable of scavenging ROS that are produced during the photoexcitation of the intratumoral photosensitizers. This reduces the effective amount of PDTproduced ROS required to induce cell death. Therefore, an extensive photochemical characterization of the compound of interest should be performed prior to further experimentation regarding pathway inhibition and PDT efficacy. Finally, when a suitable compound has been selected and has yielded favorable outcomes, a careful investigation of the prolonged antitumor immune response should be conducted. Many of the pathways discussed in this review induce immune-modulating and angiogenic factors that may negatively affect the antitumor immune response, which is required to facilitate effective removal of the tumor.

Many of the key signaling proteins discussed in this review are constitutively active in tumors and may therefore contribute to a natural resistance to PDT. Therefore, tumors that typically respond poorly to PDT such as nasopharyngeal carcinomas, bladder tumors, and extrahepatic cholangiocarcinomas may be rendered substantially more susceptible to PDT when these adaptive pathways are inhibited. Investigations regarding the constitutive activation of these pathways in the abovementioned tumor types are highly valuable in selecting a suitable pharmacological inhibition strategy.

In conclusion, the promising investigations in which survival pathway inhibitors are used as (neo)adjuvant agents in PDT are of high importance to cancer patients. A higher PDT efficacy will lead to better disease management, lower morbidity, and prolonged patient survival.

Open Access This article is distributed under the terms of the Creative Commons Attribution 4.0 International License (http:// creativecommons.org/licenses/by/4.0/), which permits unrestricted use, distribution, and reproduction in any medium, provided you give appropriate credit to the original author(s) and the source, provide a link to the Creative Commons license, and indicate if changes were made. 


\section{References}

1. Triesscheijn, M., Baas, P., Schellens, J. H. M., \& Stewart, F. A. (2006). Photodynamic therapy in oncology. The Oncologist, 11, 1034-1044.

2. Dolmans, D. E. J. G. J., Fukumura, D., \& Jain, R. K. (2003). Photodynamic therapy for cancer. Nature Reviews Cancer, 3, 380-387.

3. Grant, W. E., Speight, P. M., Hopper, C., \& B, S. G. (1997). Photodynamic therapy: an effective, but non-selective treatment for superficial cancers of the oral cavity. International Journal of Cancer, 71, 937-942.

4. Kübler, A., Niziol, C., Sidhu, M., Dünne, A., \& Werner, J. A. (2005). Analysis of cost effectiveness of photodynamic therapy with Foscan (Foscan PDT) in comparison to palliative chemotherapy in patients with advanced head-and-neck tumors in Germany. Laryngo- Rhino- Otologie, 84, 725-732.

5. Ris, H. B., Altermatt, H. J., Inderbitzi, R., Hess, R., Nachbur, B., Stewart, J. C., Wang, Q., Lim, C. K., Bonnett, R., \& B, M. C. (1991). Photodynamic therapy with chlorins for diffuse malignant mesothelioma: initial clinical results. British Journal of Cancer, $48,1273-1283$.

6. Lou, P. J., Jager, H. R., Jones, L., Theodossy, T., Bown, S. G., \& Hopper, C. (2004). Interstitial photodynamic therapy as salvage treatment for recurrent head and neck cancer. British Journal of Cancer, 91, 441-446.

7. Hopper, C., Niziol, C., \& Sidhu, M. (2004). The cost-effectiveness of Foscan mediated photodynamic therapy (Foscan-PDT) compared with extensive palliative surgery and palliative chemotherapy for patients with advanced head and neck cancer in the UK. Oral Oncology, 40, 372-382.

8. Hur, C., Nishioka, N. S., \& Gazelle, G. S. (2003). Costeffectiveness of photodynamic therapy for treatment of Barrett's esophagus with high grade dysplasia. Digestive Diseases and Sciences, 48, 1273-1283.

9. Szeimies, R. M. M. C., Sidoroff, A., \& Braathen, L. R. (2005). Photodynamic therapy for non-melanoma skin cancer. Acta Dermato-Venereologica, 85, 483-490.

10. Qumseya, B. J. D. W., \& Wolfsen, H. C. (2013). Photodynamic therapy for Barrett's esophagus and esophageal carcinoma. Clinical Endoscrophy, 46, 30-37.

11. Moghissi, K., \& Dixon, K. (2008). Update on the current indications, practice and results of photodynamic therapy (PDT) in early central lung cancer (ECLC). Photodiagnosis and Photodynamic Therapy, 5, 10-18.

12. Lee, T. Y. C. Y., \& Shim, C. S. (2013). Current status of photodynamic therapy for bile duct cancer. Clinical Endoscrophy, 46, 38 44.

13. Gomer, C.J., \& Biel, M,. Photodynamic therapy of head and neck cancers, in Photodynamic Therapy pp 281-293, Humana Press.

14. Pinthus, J. H., Bogaards, A., Weersink, R., Wilson, B. C., \& Trachtenberg, J. (2006). Photodynamic therapy for urological malignancies: past to current approaches. Journal of Urology, 175, 1201-1207.

15. Sun, Z. Q. (1992). Photodynamic therapy of nasopharyngeal carcinoma by argon or dye laser-an analysis of 137 cases. Zhonghua Zhong Liu Za Zhi, 14, 290-292.

16. Wildeman, M., Nyst, H., Karakullukcu, B., \& Tan, B. (2009). Photodynamic therapy in the therapy for recurrent/persistent nasopharyngeal cancer. Head \& Neck Oncology, 1, 40.

17. Gomer, C. J. (2012). Induction of prosurvival molecules during treatment: rethinking therapy options for photodynamic therapy. Journal of the National Comprehensive Cancer Network, 10, S-35-S-39.
18. Plaetzer, K., Krammer, B., Berlanda, J., Berr, F., \& Kiesslich, T. (2009). Photophysics and photochemistry of photodynamic therapy: fundamental aspects. Lasers in Medical Science, 24, 259-268.

19. Foote, C. S. (1991). Definition of type I and type II photosensitized oxidation. Photochemistry and Photobiology, 54, 659-659.

20. Ochsner, M. (1997). Photophysical and photobiological processes in the photodynamic therapy of tumours. Journal of Photochemistry and Photobiology B, 39, 1-18.

21. Georgiou, C. D., Papapostolou, I., Patsoukis, N., Tsegenidis, T., \& Sideris, T. (2005). An ultrasensitive fluorescent assay for the in vivo quantification of superoxide radical in organisms. Analytical Biochemistry, 347, 144-151.

22. van Golen, R. F., van Gulik, T. M., \& Heger, M. (2012). Mechanistic overview of reactive species-induced degradation of the endothelial glycocalyx during hepatic ischemia/ reperfusion injury. Free Radical Biological Medicine, 52, 1382-1402.

23. Karotki, A., Kruk, M., Drobizhev, M., Rebane, A., Nickel, E., \& Spangler, C. W. (2001). Efficient singlet oxygen generation upon two-photon excitation of new porphyrin with enhanced nonlinear absorption. IEEE Journal on Selected Topics in Quantum Electronics, 7, 971-975.

24. O'Connor, A. E., Gallagher, W. M., \& Byrne, A. T. (2009). Porphyrin and nonporphyrin photosensitizers in oncology: preclinical and clinical advances in photodynamic therapy. Photochemistry and Photobiology, 85, 1053-1074.

25. Mroz, P., Pawlak, A., Satti, M., Lee, H., Wharton, T., Gali, H., Sarna, T., \& Hamblin, M. R. (2007). Functionalized fullerenes mediate photodynamic killing of cancer cells: type I versus type II photochemical mechanism. Free Radical Biological Medicine, 43, 711-719.

26. Sakharov, D. V., Elstak, E. D. R., Chernyak, B., \& Wirtz, K. W. A. (2005). Prolonged lipid oxidation after photodynamic treatment. Study with oxidation-sensitive probe C11-BODIPY581/591. FEBS Letters, 579, 1255-1260.

27. Szokalska, A., Makowski, M., Nowis, D., Wilczynski, G. M., Kujawa, M., Wójcik, C., Mlynarczuk-Bialy, I., Salwa, P., Bil, J., Janowska, S., Agostinis, P., Verfaillie, T., Bugajski, M., Gietka, J., Issat, T., Glodkowska, E., Mrówka, P., Stoklosa, T., Hamblin, M. R., Mróz, P., Jakóbisiak, M., \& Golab, J. (2009). Proteasome inhibition potentiates antitumor effects of photodynamic therapy in mice through induction of endoplasmic reticulum stress and unfolded protein response. Cancer Research, 69, 4235-4243.

28. Chiou, J. F., Wang, Y. H., Jou, M. J., Liu, T. Z., \& Shiau, C. Y. (2009). Verteporfin-photoinduced apoptosis in HepG2 cells mediated by reactive oxygen and nitrogen species intermediates. Free Radical Research, 44, 155-170.

29. Cadet, J., Douki, T., \& Ravanat, J.-L. (2008). Oxidatively generated damage to the guanine moiety of DNA: mechanistic aspects and formation in cells. Accounts of Chemical Research, 41, 1075-1083.

30. Blazek, E. R., \& Hariharan, P. V. (1984). Alkaline elution studies of hematoporphyrin-derivative photosensitized DNA damage and repair in Chinese hamster ovary cells. Photochemistry and Photobiology, 40, 5-13.

31. Boegheim, J. P. J., Scholte, H., Dubbelman, T. M. A. R., Beems, E., Raap, A. K., \& Van Steveninck, J. (1987). Photodynamic effects of hematoporphyrin-derivative on enzyme activities of murine L929 fibroblasts. Journal of Photochemistry and Photobiology B, 1, 61-73.

32. Fiel, R. J., Datta-Gupta, N., Mark, E. H., \& Howard, J. C. (1981). Induction of DNA damage by porphyrin photosensitizers. Cancer Research, 41, 3543-3545. 
33. Kessel, D., \& Sun, H. H. (1999). Enhanced responsiveness to photodynamic therapy-induced apoptosis after mitochondrial DNA depletion. Photochemistry and Photobiology, 70, 937-940.

34. Kvam, E., \& Moan, J. (1990). A comparison of three photosensitizers with respect to efficiency of cell inactivation, fluorescence quantum yield and DNA strand breaks. Photochemistry and Photobiology, 52, 769-773.

35. McNair, F. I., Marples, B., West, C. M., \& Moore, J. V. (1997). A comet assay of DNA damage and repair in K562 cells after photodynamic therapy using haematoporphyrin derivative, methylene blue and meso-tetrahydroxyphenylchlorin. British Journal of Cancer, 75, 1721-1729.

36. Penning, L. C., Lagerberg, J. W. M., VanDierendonck, J. H., Cornelisse, C. J., Dubbelman, T. M. A. R., \& VanSteveninck, J. (1994). The role of DNA damage and inhibition of poly(ADPribosyl)ation in loss of clonogenicity of murine L929 fibroblasts, caused by photodynamically induced oxidative stress. Cancer Research, 54, 5561-5567.

37. Penning, L. C., Tijssen, K., Boegheim, J. P. J., van Steveninck, J., \& Dubbelman, T. M. A. R. (1994). Relationship between photodynamically induced damage to various cellular parameters and loss of clonogenicity in different cell types with hematoporphyrin derivative as sensitizer. Biochimica et Biophysica Acta, 1221, 250-258.

38. Rousset, N., Kerninon, E., Eléouet, S., Le Néel, T., Auget, J.-L., Vonarx, V., Carré, J., Lajat, Y., \& Patrice, T. (2000). Use of alkaline Comet assay to assess DNA repair after mTHPC-PDT. Journal of Photochemistry and Photobiology $B, 56,118-131$.

39. Nunomura, A., Honda, K., Takeda, A., Hirai, K, Zhu, X.W., Smith, M.A., \& Perry, G. (2006). Oxidative damage to RNA in neurodegenerative diseases. Journal of Biomedicine Biotechnology: 6.

40. Shan, X., Chang, Y., \& Lin, C. G. (2007). Messenger RNA oxidation is an early event preceding cell death and causes reduced protein expression. FASEB Journal, 21, 2753-2764.

41. Niki, E. (2009). Lipid peroxidation: physiological levels and dual biological effects. Free Radical Biological Medicine, 47, 469-484.

42. Minami, Y., Yokoyama, K., Bando, N., Kawai, Y., \& Terao, J. (2009). Occurrence of singlet oxygen oxygenation of oleic acid and linoleic acid in the skin of live mice. Free Radical Research, 42, 197-204.

43. Murphy, R. C., \& Johnson, K. M. (2008). Cholesterol, reactive oxygen species, and the formation of biologically active mediators. Journal of Biological Chemistry, 283, 15521-15525.

44. Ouchi, A., Ishikura, M., Konishi, K., Nagaoka, S., \& Mukai, K. (2009). Kinetic study of the prooxidant effect of $\alpha$-tocopherol. Hydrogen abstraction from lipids by $\alpha$-tocopheroxyl radical. Lipids, 44, 935-943.

45. van Reyk, D. M., Brown, A. J., Hult'en, L. M., Dean, R. T., \& Jessup, W. (2006). Oxysterols in biological systems: sources, metabolism and pathophysiological relevance. Redox Report, 11, 255-262.

46. Vejux, A., Malvitte, L., \& Lizard, G. (2008). Side effects of oxysterols: cytotoxicity, oxidation, inflammation, and phospholipidosis. Brazilian Journal of Medical and Biological Research, 41, 545-556.

47. Borchman, D., Lamba, O., Salmassi, S., Lou, M., \& Yappert, M. C. (1992). The dual effect of oxidation on lipid bilayer structure. Lipids, 27, 261-265.

48. Catalá, A. (2009). Lipid peroxidation of membrane phospholipids generates hydroxy-alkenals and oxidized phospholipids active in physiological and/or pathological conditions. Chemistry and Physics of Lipids, 157, 1-11.
49. Chatterjee, S. N., \& Agarwal, S. (1988). Liposomes as membrane model for study of lipid peroxidation. Free Radical Biological Medicine, 4, 51-72.

50. Dinis, T. C. P., Almeida, L. M., \& Madeira, V. M. C. (1993). Lipid peroxidation in sarcoplasmic reticulum membranes: effect on functional and biophysical properties. Archives of Biochemistry and Biophysics, 301, 256-264.

51. Goel, R., Mishra, O. P., Razdan, B., \& DelivoriaPapadopoulos, M. (1993). Modification of NMDA receptor by in vitro lipid peroxidation in fetal guinea pig brain. Neuroscience Letters, 151, 219-223.

52. Goldstein, I. M., \& Weissmann, G. (1977). Effects of the generation of superoxide anion on permeability of liposomes. Biochemical and Biophysical Research Communications, 75, 604-609.

53. Kourie, J. I. (1998). Interaction of reactive oxygen species with ion transport mechanisms. American Journal of Physiology, 275, C124.

54. Kunimoto, M., Inoue, K., \& Nojima, S. (1981). Effect of ferrous ion and ascorbate-induced lipid peroxidation on liposomal membranes. Biochimica et Biophysica Acta, 646, 169-178.

55. Mattson, M. P. (1998). Modification of ion homeostasis by lipid peroxidation: roles in neuronal degeneration and adaptive plasticity. Trends in Neurosciences, 21, 53-57.

56. Mattson, M. P., Pedersen, W. A., Duan, W., Culmsee, C., \& Camandola, S. (1999). Cellular and molecular mechanisms underlying perturbed energy metabolism and neuronal degeneration in Alzheimer's and Parkinson's diseases. Annals of the New York Academy of Sciences, 893, 154-175.

57. Petrescu, A. D., Gallegos, A. M., Okamura, Y., Strauss, J. F., \& Schroeder, F. (2001). Steroidogenic acute regulatory protein binds cholesterol and modulates mitochondrial membrane sterol domain dynamics. Journal of Biological Chemistry, 276, 36970-36982.

58. Sevanian, A., \& Ursini, F. (2000). Lipid peroxidation in membranes and low-density lipoproteins: similarities and differences. Free Radical Biological Medicine, 29, 306-311.

59. Van Duijn, G., Verkleij, A. J., \& De Kruijff, B. (1984). Influence of phospholipid peroxidation on the phase behavior of phosphatidylcholine and phosphatidylethanolamine in aqueous dispersions. Biochemistry, 23, 4969-4977.

60. Yukawa, O., Nagatsuka, S., \& Nakazawa, T. (2009). Reconstitution studies on the involvement of radiation-induced lipid peroxidation in damage to membrane enzymes. International Journal of Radiation Biology, 43, 391-398.

61. Stadtman, E. R. (2009). Protein oxidation and aging. Free Radical Research, 40, 1250-1258.

62. Tsaytler, P. A., O'Flaherty, M. C., Sakharov, D. V., Krijgsveld, J., \& Egmond, M. R. (2008). Immediate protein targets of photodynamic treatment in carcinoma cells. Journal of Proteome Research, 7, 3868-3878.

63. Castano, A. P., Demidova, T. N., \& Hamblin, M. R. (2005). Mechanisms in photodynamic therapy: part three-photosensitizer pharmacokinetics, biodistribution, tumor localization and modes of tumor destruction. Photodiagnosis and Photodynamic Therapy, 2, 91-106.

64. Coupienne, I., Fettweis, G., Rubio, N., Agostinis, P., \& Piette, J. (2011). 5-ALA-PDT induces RIP3-dependent necrosis in glioblastoma. Photochemistry \& Photobiological Sciences, 10, 1868-1878.

65. Dougherty, T. J., Gomer, C. J., Henderson, B. W., Jori, G., Kessel, D., Korbelik, M., Moan, J., \& Peng, Q. (1998). Photodynamic therapy. Journal of the National Cancer Institute, 90, 889-905.

66. Reiners, J. J., Agostinis, P., Berg, K., Oleinick, N. L., \& Kessel, D. H. (2010). Assessing autophagy in the context of photodynamic therapy. Autophagy, 6, 7-18. 
67. Weston, M. A., \& Patterson, M. S. (2014). Measurement of intracellular oxygen concentration during photodynamic therapy in vitro. Photochemistry and Photobiology, 90, 878-888.

68. Fingar, V. H. (1996). Vascular effects of photodynamic therapy. Journal of Clinical Laser Medicine and Surgery, 14, 323-328.

69. Chen, B., Pogue, B. W., Hoopes, P. J., \& Hasan, T. (2005). Combining vascular and cellular targeting regimens enhances the efficacy of photodynamic therapy. International Journal of Radiation Oncology Biology Physics, 61, 1216-1226.

70. Triesscheijn, M. R. M., Aalders, M., Baas, P., \& Stewart, F. A. (2005). Outcome of mTHPC mediated photodynamic therapy is primarily determined by the vascular response. Photochemistry and Photobiology, 81, 1161-1167.

71. Fingar, V. H., Kik, P. K., Haydon, P. S., Cerrito, P. B., Tseng, M., Abang, E., \& Wieman, T. J. (1999). Analysis of acute vascular damage after photodynamic therapy using benzoporphyrin derivative (BPD). British Journal of Cancer, 79, 1702-1708.

72. Fingar, V. H., Wieman, T. J., Karavolos, P. S., Doak, K. W., Ouellet, R., \& van Lier, J. E. (1993). The effects of photodynamic therapy using differently substituted zinc phthalocyanines on vessel constriction, vessel leakage and tumor response. Photochemistry and Photobiology, 58, 251-258.

73. Fingar, V. H., Wieman, T. J., Wiehle, S. A., \& Cerrito, P. B. (1992). The role of microvascular damage in photodynamic therapy: the effect of treatment on vessel constriction, permeability, and leukocyte adhesion. Cancer Research, 52, 4914-4921.

74. Roberts, D. J. H., Cairnduff, F., Driver, I., Dixon, B., \& Brown, S. B. (1994). Tumor vascular shutdown following photodynamic therapy based on polyhematoporphyrin or 5-aminolevulinic acid. International Journal of Oncology, 5, 763-768.

75. Hilf, R. (2007). Mitochondria are targets of photodynamic therapy. Journal of Bioenergetics and Biomembranes, 39, 85-89.

76. Korbelik, M., \& Cecic, I. (1999). Contribution of myeloid and lymphoid host cells to the curative outcome of mouse sarcoma treatment by photodynamic therapy. Cancer Letters, 137, 91-98.

77. Korbelik, M., Krosl, G., Krosl, J., \& Dougherty, G. J. (1996). The role of host lymphoid populations in the response of mouse EMT6 tumor to photodynamic therapy. Cancer Research, 56, 56475652.

78. Broekgaarden, M., de Kroon, A. I. P. M., van Gulik, T. M., \& Heger, M. (2013). Development and in vitro proof-of-concept of interstitially targeted zinc-phthalocyanine liposomes for photodynamic therapy. Current Medicinal Chemistry, 21, 377-391.

79. Garg, A.D., Krysko, D.V., Vandenabeele P \& Agostinis, P. (2011). DAMPs and PDT-mediated photo-oxidative stress: exploring the unknown. Photochemical \& Photobiological Sciences 10:670-680.

80. Chen, G. Y., \& Nuñez, G. (2010). Sterile inflammation: sensing and reacting to damage. Nature Reviews Immunology, 10, 826837.

81. Mroz, P., Hashmi, J. T., Huang, Y.-Y., Lange, N., \& Hamblin, M. R. (2011). Stimulation of anti-tumor immunity by photodynamic therapy. Experimental Review on Clinical Immunology, 7, 75-91.

82. Krysko, D. V., Garg, A. D., Kaczmarek, A., Krysko, O., Agostinis, P., \& Vandenabeele, P. (2012). Immunogenic cell death and DAMPs in cancer therapy. Nature Reviews Cancer, 12, 860-875.

83. Kabingu, E., Vaughan, L., Owczarczak, B., Ramsey, K. D., \& Gollnick, S. O. (2007). CD8+ T cell-mediated control of distant tumours following local photodynamic therapy is independent of CD4+ T cells and dependent on natural killer cells. British Journal of Cancer, 96, 1839-1848.

84. Thong, P. S.-P., Ong, K. W., Goh, N. S. G., Kho, K. W., Manivasager, V., Bhuvaneswari, R., Olivo, M., \& Soo, K. C. (2007). Photodynamic-therapy-activated immune response against distant untreated tumours in recurrent angiosarcoma. Lancet Oncology, 8, 950-952.

85. Kocanova, S., Buytaert, E., Matroule, J.-Y., Piette, J., Golab, J., de Witte, P., \& Agostinis, P. (2007). Induction of heme-oxygenase 1 requires the p38MAPK and PI3K pathways and suppresses apoptotic cell death following hypericin-mediated photodynamic therapy. Apoptosis, 12, 731-741.

86. Li, W., \& Kong, A. N. (2009). Molecular mechanisms of Nrf2mediated antioxidant response. Molecular Carcinogenesis, 48, 91-104.

87. Motohashi, H., \& Yamamoto, M. (2004). Nrf2-Keap1 defines a physiologically important stress response mechanism. Trends in Molecular Medicine, 10, 549-557.

88. Hayes, J. D. M., Chowdhry, S. M., \& Dinkova-Kostova, A. T. (2010). Cancer chemoprevention mechanisms mediated through the Keap1-Nrf2 pathway. Antioxidants and Redox Signaling, 13, 1713-1748.

89. Na, H. K., \& Surh, Y. J. (2014). Oncogenic potential of Nrf2 and its principal target protein heme oxygenase-1. Free Radical Biological Medicine, 67, 353-365.

90. Gañán-Gómez, I., Wei, Y., Yang, H., Boyano-Adánez, M. C., \& García-Manero, G. (2013). Oncogenic functions of the transcription factor Nrf2. Free Radical Biological Medicine, 65, 750-764.

91. Chan, J. Y., Han, X. L., \& Kan, Y. W. (1993). Isolation of cDNAencoding the human NF-E2 protein. Proceedings of National Academy of Sciences U S A, 90, 11366-11370.

92. McMahon, M., Itoh, K., Yamamoto, M., Chanas, S. A., Henderson, C. J., McLellan, L. I., Wolf, C. R., Cavin, C., \& Hayes, J. D. (2001). The cap 'n' collar basic leucine zipper transcription factor Nrf2 (NF-E2 p45-related factor 2) controls both constitutive and inducible expression of intestinal detoxification and glutathione biosynthetic enzymes. Cancer Research, 61, 3299-3307.

93. Moi, P., Chan, K., Asunis, I., Cao, A., \& Kan, Y. W. (1994). Isolation of NF-E2-related factor-2 (NRF2), and NF-E2-like basic leucine-zipper transcriptional activator that binds to the tandem NF-E2/AP1 repeat of the beta-globin locus control region. Proceedings of National Academy of Sciences U S A, 91, 9926 9930.

94. Itoh, K., Wakabayashi, N., Katoh, Y., Ishii, T., Igarashi, K., Engel, J. D., \& Yamamoto, M. (1999). Keap1 represses nuclear activation of antioxidant responsive elements by $\mathrm{Nrf} 2$ through binding to the amino-terminal Neh2 domain. Genes and Development, 13, 76-86.

95. Zipper, L. M., \& Mulcahy, R. T. (2002). The keap1 BTB/POZ dimerization function is required to sequester $\mathrm{Nrf} 2$ in cytoplasm. Journal of Biological Chemistry, 277, 36544-36552.

96. Zhang, D. D., \& Hannink, M. (2003). Distinct cysteine residues in keap1 are required for keap1-dependent ubiquitination of Nrf2 and for stabilization of Nrf2 by chemopreventive agents and oxidative stress. Molecular and Cellular Biology, 23, 8137-8151.

97. Bloom, D. A., \& Jaiswal, A. K. (2003). Phosphorylation of Nrf2 at Ser40 by protein kinase $\mathrm{C}$ in response to antioxidants leads to the release of $\mathrm{Nrf2}$ from INrf2, but is not required for $\mathrm{Nrf2}$ stabilization/accumulation in the nucleus and transcriptional activation of antioxidant response element-mediated NAD(P)H:quinone oxidoreductase-1 gene expression. Journal of Biological Chemistry, 278, 44675-44682.

98. Copple, I. M., Goldring, C. E., Kitteringham, N. R., \& Park, B. K. (2008). The Nrf2-Keap1 defence pathway: role in protection against drug-induced toxicity. Toxicology, 246, 24-33.

99. Huang, H. C., Nguyen, T., \& Pickett, C. B. (2002). Phosphorylation of Nrf2 at Ser-40 by protein kinase C regulates antioxidant response element-mediated transcription. Journal of Biological Chemistry, 277, 42769-42774. 
100. Itoh, K., Chiba, T., Takahashi, S., Ishii, T., Igarashi, K., Katoh, Y., Oyake, T., Hayashi, N., Satoh, K., Hatayama, I., Yamamoto, M., \& Nabeshima, Y. (1997). An Nrf2 small Maf heterodimer mediates the induction of phase II detoxifying enzyme genes through antioxidant response elements. Biochemical and Biophysical Research Communications, 236, 313-322.

101. Venugopal, R., \& Jaiswal, A. K. (1998). Nrf2 and Nrf1 in association with Jun proteins regulate antioxidant response elementmediated expression and coordinated induction of genes encoding detoxifying enzymes. Oncogene, 17, 3145-3156.

102. Klaassen, C. D., \& Reisman, S. A. (2010). Nrf2 the rescue: effects of the antioxidative/electrophilic response on the liver. Toxicology and Applied Pharmacology, 244, 57-65.

103. Ross, D. (2004). Quinone reductases multitasking in the metabolic world. Drug Metabolism Reviews, 36, 639-654.

104. Widersten, M., Gurell, A., \& Lindberg, D. (2010). Structurefunction relationships of epoxide hydrolases and their potential use in biocatalysis. Biochimica et Biophysica Acta, 1800, 316326.

105. Satoh, T., \& Hosokawa, M. (1998). The mammalian carboxylesterases: from molecules to functions. Annual Review Pharmacological Toxicology, 38, 257-288.

106. Gozzelino, R., Jeney, V., \& Soares, M. P. (2010). Mechanisms of cell protection by heme oxygenase-1. Annual Review on Pharmacological Toxicology, 50, 323-354.

107. Kloek, J. J., Marechal, X., Roelofsen, J., Houtkooper, R. H., van Kuilenburg, A. B., Kulik, W., Bezemer, R., Neviere, R., van Gulik, T. M., \& Heger, M. (2012). Cholestasis is associated with hepatic microvascular dysfunction and aberrant energy metabolism before and during ischemia-reperfusion. Antioxidants and Redox Signaling, 17, 1109-1123.

108. Jeong, W., Park, S. J., Chang, T. S., Lee, D. Y., \& Rhee, S. G. (2006). Molecular mechanism of the reduction of cysteine sulfinic acid of peroxiredoxin to cysteine by mammalian sulfiredoxin. Journal of Biological Chemistry, 281, 14400-14407.

109. Lu, S. C. (2009). Regulation of glutathione synthesis. Molecular Aspects of Medicine, 30, 42-59.

110. Borst, P., Evers, R., Kool, M., \& Wijnholds, J. (2000). A family of drug transporters: the multidrug resistance-associated proteins. Journal of the National Cancer Institute, 92, 1295-1302.

111. Hagiya, Y., Adachi, T., Ogura, S., An, R., Tamura, A., Nakagawa, H., Okura, I., Mochizuki, T., \& Ishikawa, T. (2008). NRF2dependent induction of human $\mathrm{ABC}$ transporter $\mathrm{ABCG} 2$ and heme oxygenase-1 in HepG2 cells by photoactivation of porphyrins: biochemical implications for cancer cell response to photodynamic therapy. Journal of Experimental Therapeutics and Oncology, 7, 153-167.

112. Singh, A., Wu, H., Zhang, P., Happel, C., Ma, J., \& Biswal, S. (2010). Expression of ABCG2 (BCRP) is regulated by Nrf2 in cancer cells that confers side population and chemoresistance phenotype. Molecular Cancer Therapeutics, 9, 2365-2376.

113. Ockaili, R., Natarajan, R., Salloum, F., Fisher, B. J., Jones, D., Fowler, A. A., \& Kukreja, R. C. (2005). HIF-1 activation attenuates postischemic myocardial injury: role for heme oxygenase- 1 in modulating microvascular chemokine generation. American Journal of Physiology, 289, H542-H548.

114. Kapitulnik, J., \& Maines, M. D. (2009). Pleiotropic functions of biliverdin reductase: cellular signaling and generation of cytoprotective and cytotoxic bilirubin. Trends in Pharmacological Sciences, 30, 129-137.

115. Dudnik, L. B., \& Khrapova, N. G. (1998). Characterization of bilirubin inhibitory properties in free radical oxidation reactions. Membrane Cell Biology, 12, 233-240.

116. Kapitulnik, J. (2004). Bilirubin: an endogenous product of heme degradation with both cytotoxic and cytoprotective properties. Molecular Pharmacology, 66, 773-779.
117. Dulak, J., \& Józkowicz, A. (2003). Carbon monoxide-a "new" gaseous modulator of gene expression. Acta Biochimica Polonica, 50, 31-47.

118. Ryter, S. W., \& Otterbein, L. E. (2004). Carbon monoxide in biology and medicine. Bioessays, 26, 270-280.

119. Bauer, M., \& Bauer, A. (2002). Heme oxygenase-1: redox regulation and role in the hepatic response to oxidative stress. Antioxidants and Redox Signaling, 4, 749-758.

120. Arosio, P., \& Levi, S. (2002). Ferritin, iron homeostasis, and oxidative damage. Free Radiation Biological Medicine, 33, $457-$ 463.

121. Circu, M. L., \& Yee Aw, T. (2008). Glutathione and apoptosis. Free Radical Research, 42, 689-706.

122. Tew, K. D., \& Townsend, D. M. (2012). Glutathione-Stransferases as determinants of cell survival and death. Antioxidants and Redox Signaling, 17, 1728-1737.

123. Nowis, D., Legat, M., Grzela, T., Niderla, J., Wilczek, E., Wilczynski, G. M., Glodkowska, E., Mrowka, P., Issat, T., \& Dulak, J. (2006). Heme oxygenase-1 protects tumor cells against photodynamic therapy-mediated cytotoxicity. Oncogene, 25, 3365-3374.

124. Buytaert, E., Matroule, J. Y., Durinck, S., Close, P., Kocanova, S., Vandenheede, J. R., de Witte, P. A., Piette, J., \& Agostinis, P. (2007). Molecular effectors and modulators of hypericinmediated cell death in bladder cancer cells. Oncogene, 27, 1916-1929.

125. Lamberti, M. J., Rumie Vittar, N. B., de Carvalho da Silva, F., Ferreira, V. F., \& Rivarola, V. A. (2013). Synergistic enhancement of antitumor effect of $\beta$-Lapachone by photodynamic induction of quinone oxidoreductase (NQO1). Phytomedicine, 20, 1007-1012.

126. Frank, J., Lornejad-Schäfer, M. R., Schöffl, H., Flaccus, A., Lambert, C., \& Biesalski, H. K. (2007). Inhibition of heme oxygenase-1 increases responsiveness of melanoma cells to ALA-based photodynamic therapy. International Journal of Oncology, 31, 1539-1545.

127. Yoon, J.-H., Yoon, H.-E., Kim, O., Kim, S. K., Ahn, S.-G., \& Kang, K. W. (2012). The enhanced anti-cancer effect of hexenyl ester of 5-aminolaevulinic acid photodynamic therapy in adriamycin-resistant compared to non-resistant breast cancer cells. Lasers in Surgery and Medicine, 44, 76-86.

128. Miyake, M., Ishii, M., Kawashima, K., Kodama, T., Sugano, K., Fujimoto, K., \& Hirao, Y. (2009). siRNA-mediated knockdown of the heme synthesis and degradation pathways: modulation of treatment effect of 5-aminolevulinic acid-based photodynamic therapy in urothelial cancer cell lines. Photochemistry and Photobiology, 85, 1020-1027.

129. Liu, W., Baer, M. R., Bowman, M. J., Pera, P., Zheng, X., Morgan, J., Pandey, R. A., \& Oseroff, A. R. (2007). The tyrosine kinase inhibitor imatinib mesylate enhances the efficacy of photodynamic therapy by inhibiting ABCG2. Clinical Cancer Research, 13, 2463-2470.

130. Robey, R. W., Steadman, K., Polgar, O., \& Bates, S. E. (2005). ABCG2-mediated transport of photosensitizers: potential impact on photodynamic therapy. Cancer Biology and Therapy, 4, 195 202.

131. Wang, X. J., Hayes, J. D., Henderson, C. J., \& Wolf, C. R. (2007). Identification of retinoic acid as an inhibitor of transcription factor Nrf2 through activation of retinoic acid receptor alpha. Proceedings of National Academy of Sciences U S A, 104, 19589-19594.

132. Labbé, R. F., Vreman, H. J., \& Stevenson, D. K. (1999). Zinc protoporphyrin: a metabolite with a mission. Clinical Chemist, 45, 2060-2072.

133. Drummond, G. S., \& Kappas, A. (1981). Prevention of neonatal hyperbilirubinemia by tin protoporphyrin IX, a potent competitive 
inhibitor of heme oxidation. Proceedings of the National Academy of Sciences of the United States of America, 78, 6466-6470.

134. Kachadourian, R., Liochev, S. I., Cabelli, D. E., Patel, M. N., Fridovich, I., \& Day, B. J. (2001). 2-Methoxyestradiol does not inhibit superoxide dismutase. Archives of Biochemistry and Biophysics, 392, 349-353.

135. Griffith, O. W. (1982). Mechanism of action, metabolism, and toxicity of buthionine sulfoximine and its higher homologs, potent inhibitors of glutathione synthesis. Journal of Biological Chemistry, 257, 13704-13712.

136. Ueda, M., Kinoshita, H., Yoshida, T., Kamasawa, N., Osumi, M., \& Tanaka, A. (2003). Effect of catalase-specific inhibitor 3-amino1,2,4-triazole on yeast peroxisomal catalase in vivo. FEMS Microbiology Letters, 219, 93-98.

137. García-Piñeres, A. J., Castro, V., Mora, G., Schmidt, T. J., Strunck, E., Pahl, H. L., \& Merfort, I. (2001). Cysteine 38 in p65/NFkappaB plays a crucial role in DNA binding inhibition by sesquiterpene lactones. Journal of Biological Chemistry, 276, 3971339720.

138. Erkel, G., Anke, T., \& Sterner, O. (1996). Inhibition of NF-kB activation by panepoxydone. Biochemical and Biophysical Research Communications, 226, 214-221.

139. Lee, J., Rhee, M. H., Kim, E., \& Cho, J. Y. (2012). BAY 11-7082 is a broad-spectrum inhibitor with anti-inflammatory activity against multiple targets. Mediators of Inflammation, 2012, 11.

140. Nishimura, D., Ishikawa, H., Matsumoto, K., Shibata, H., Motoyoshi, Y., Fukuta, M., Kawashimo, H., Goto, T., Taura, N., Ichikawa, T., Hamasaki, K., Nakao, K., Umezawa, K., \& Eguchi, K. (2006). DHMEQ, a novel NF-kB inhibitor, induces apoptosis and cell-cycle arrest in human hepatoma cells. International Journal of Oncology, 29, 713-719.

141. MacKenzie, E. D., Selak, M. A., Tennant, D. A., Payne, L. J., Crosby, S., Frederiksen, C. M., Watson, D. G., \& Gottlieb, E. (2007). Cell-permeating $\alpha$-ketoglutarate derivatives alleviate pseudohypoxia in succinate dehydrogenase-deficient cells. Molecular and Cellular Biology, 27, 3282-3289.

142. Hawkey, C. J. (1999). COX-2 inhibitors. Lancet, 353, 307-314.

143. Song, H., Wang, R., Wang, S., \& Lin, J. (2005). A low-molecularweight compound discovered through virtual database screening inhibits Stat3 function in breast cancer cells. Proceedings of the National Academy of Sciences of the United States of America, $102,4700-4705$.

144. Iwamaru, A., Szymanski, S., Iwado, E., Aoki, H., Yokoyama, T., Fokt, I., Hess, K., Conrad, C., Madden, T., Sawaya, R., Kondo, S., Priebe, W., \& Kondo, Y. (2007). A novel inhibitor of the STAT3 pathway induces apoptosis in malignant glioma cells both in vitro and in vivo. Oncogene, 26, 2435-2444.

145. Altieri, D. C. (2008). Survivin, cancer networks and pathwaydirected drug discovery. Nature Reviews Cancer, 8, 61-70.

146. Rose-John, S., Waetzig, G. H., Scheller, J., Grötzinger, J., \& Seegert, D. (2007). The IL-6/sIL-6R complex as a novel target for therapeutic approaches. Expert Opinion on Therapeutic Targets, 11, 613-624.

147. Brown, P. (1997). Matrix metalloproteinase inhibitors in the treatment of cancer. Medical Oncology, 14, 1-10.

148. Yeo, E. J., Ryu, J. H., Cho, Y. S., Chun, Y. S., Huang, L. E., Kim, M. S., \& Park, J. W. (2006). Amphotericin B blunts erythropoietin response to hypoxia by reinforcing $\mathrm{FIH}$-mediated repression of HIF-1. Blood, 107, 916-923.

149. Kong, D., Park, E. J., Stephen, A. G., Calvani, M., Cardellina, J. H., Monks, A., Fisher, R. J., Shoemaker, R. H., \& Melillo, G. (2005). Echinomycin, a small-molecule inhibitor of hypoxiainducible factor-1 DNA-binding activity. Cancer Research, 65, 9047-9055.

150. Choi, H., Chun, Y. S., Kim, S. W., Kim, M. S., \& Park, J. W. (2006). Curcumin inhibits hypoxia-inducible factor-1 by degrading aryl hydrocarbon receptor nuclear translocator: a mechanism of tumor growth inhibition. Molecular Pharmacology, 70, 1664-1671.

151. Lee, K., Zhang, H., Qian, D. Z., Rey, S., Liu, J. O., \& Semenza, G. L. (2009). Acriflavine inhibits HIF-1 dimerization, tumor growth, and vascularization. Proceedings of the National Academy of Sciences of the United States of America, 106, 17910-17915.

152. Huang, C., Ma, W. Y., Dawson, M. I., Rincon, M., Flavell, R. A., \& Dong, Z. (1997). Blocking activator protein-1 activity, but not activating retinoic acid response element, is required for the antitumor promotion effect of retinoic-acid. Proceedings of National Academy of Sciences U S A, 94, 5826-5830.

153. Bennett, B. L., Sasaki, D. T., Murray, B. W., O’Leary, E. C., Sakata, S. T., Xu, W., Leisten, J. C., Motiwala, A., Pierce, S., Satoh, Y., Bhagwat, S. S., Manning, A. M., \& Anderson, D. W. (2001). SP600125, an anthrapyrazolone inhibitor of Jun Nterminal kinase. Proceedings of the National Academy of Sciences of the United States of America, 98, 13681-13686.

154. Gallagher, T. F., Seibel, G. L., Kassis, S., Laydon, J. T., Blumenthal, M. J., Lee, J. C., Lee, D., Boehm, J. C., FierThompson, S. M., Abt, J. W., Soreson, M. E., Smietana, J. M., Hall, R. F., Garigipati, R. S., Bender, P. E., Erhard, K. F., Krog, A. J., Hofmann, G. A., Sheldrake, P. L., McDonnell, P. C., Kumar, S., Young, P. R., \& Adams, J. L. (1997). Regulation of stress-induced cytokine production by pyridinylimidazoles; inhibition of CSBP kinase. Bioorganic and Medicinal Chemistry, 5, 49-64.

155. Hadden, K. M., Lubbers, D. J., \& Blagg, B. S. J. (2006). Geldanamycin, radicicol, and chimeric inhibitors of the Hsp90 $\mathrm{N}$-terminal ATP binding site. Current Topics in Medicinal Chemistry, 6, 1173-1182.

156. Lundgren, K., Zhang, H., Brekken, J., Huser, N., Powell, R. E., Timple, N., Busch, D. J., Neely, L., Sensintaffar, J. L., Yang, Y.-c., McKenzie, A., Friedman, J., Scannevin, R., Kamal, A., Hong, K., Kasibhatla, S. R., Boehm, M. F., \& Burrows, F. J. (2009). BIIB021, an orally available, fully synthetic small-molecule inhibitor of the heat shock protein Hsp90. Molecular Cancer Therapeutics, 8, 921-929.

157. Lee, K.-H., Lee, J.-H., Han, S.-W., Im, S.-A., Kim, T.-Y., Oh, D.Y., \& Bang, Y.-J. (2011). Antitumor activity of NVP-AUY922, a novel heat shock protein 90 inhibitor, in human gastric cancer cells is mediated through proteasomal degradation of client proteins. Cancer Science, 102, 1388-1395.

158. Chandarlapaty, S., Sawai, A., Ye, Q., Scott, A., Silinski, M., Huang, K., Fadden, P., Partdrige, J., Hall, S., Steed, P., Norton, L., Rosen, N., \& Solit, D. B. (2008). SNX2112, a synthetic heat shock protein 90 inhibitor, has potent antitumor activity against HER kinase-dependent cancers. Clinical Cancer Research, 14, 240-248.

159. Wang, Y., Trepel, J. B., Neckers, L. M., \& Giaccone, G. (2010). STA-9090, a small-molecule Hsp90 inhibitor for the potential treatment of cancer. Current Opinion in Investigational Drugs, $11,1466-1476$.

160. Backer, J., Krivoshein, A. V., Hamby, C. V., Pizzonia, J., GK, S., Ray, Y. S., Brand, H., Paton, A. W., Paton, J. C., \& Backer, M. V. (2009). Chaperone-targeting cytotoxin and endoplasmic reticulum stress-inducing drug synergize to kill cancer cells. Neoplasia, 11, $1165-1173$.

161. Schlecht, R., Scholz, S. R., Dahmen, H., Wegener, A., Sirrenberg, C., Musil, D., Bomke, J., Eggenweiler, H.-M., Mayer, M. P., \& Bukau, B. (2013). Functional analysis of Hsp70 inhibitors. PLoS ONE, 8, e78443.

162. Bonvini, P., Zorzi, E., Basso, G., \& Rosolen, A. (2007). Bortezomib-mediated 26S proteasome inhibition causes cellcycle arrest and induces apoptosis in CD-30+ anaplastic large cell lymphoma. Leukemia, 21, 838-842. 
163. Zhang, H., Nakajima, S., Kato, H., Gu, L., Yoshitomi, T., Nagai, K., Shinmori, H., Kokubo, S., \& Kitamura, M. (2013). Selective, potent blockade of the IRE1 and ATF6 pathways by 4phenylbutyric acid analogues. British Journal of Pharmacology, 170, 822-834.

164. Atkins, C., Liu, Q., Minthorn, E., Zhang, S.-Y., Figueroa, D. J., Moss, K., Stanley, T. B., Sanders, B., Goetz, A., Gaul, N., Choudhry, A. E., Alsaid, H., Jucker, B. M., Axten, J. M., \& Kumar, R. (2013). Characterization of a novel PERK kinase inhibitor with antitumor and antiangiogenic activity. Cancer Research, 73, 1993-2002.

165. Maines, M. D. (1981). Zinc-protoporphyrin is a selective inhibitor of heme oxygenase activity in the neonatal rat. Biochimica et Biophysica Acta, 673, 339-350.

166. Kimani, S. G., Phillips, J. B., Bruce, J. I., MacRobert, A. J., \& Golding, J. P. (2012). Antioxidant inhibitors potentiate the cytotoxicity of photodynamic therapy. Photochemistry and Photobiology, 88, 175-187.

167. Misra, H. P. (1979). Reaction of copper-zinc superoxide dismutase with diethyldithiocarbamate. Journal of Biological Chemistry, 254, 11623-11628.

168. Taylor, C. T., \& Cummins, E. P. (2009). The role of NF-kappa B in hypoxia-induced gene expression. Annals of the New York Academy of Sciences, 1177, 178-184.

169. Matroule, J.-Y., Volanti, C., \& Piette, J. (2006). NF-kB in photodynamic therapy: discrepancies of a master regulator. Photochemistry and Photobiology, 82, 1241-1246.

170. Song, J., Wei, Y., Chen, Q., \& Xing, D. (2014). Cyclooxygenase 2-mediated apoptotic and inflammatory responses in photodynamic therapy treated breast adenocarcinoma cells and xenografts. Journal of Photochemistry and Photobiology B, 134, 27-36.

171. Ghosh, S., May, M. J., \& Kopp, E. B. (2003). NF-kB and REL proteins: evolutionarily conserved mediators of immune responses. Annual Review on Immunology, 16, 225-260.

172. Karin, M., Cao, Y., Greten, F. R., \& Li, Z.-W. (2002). NF-kappaB in cancer: from innocent bystander to major culprit. Nature Reviews Cancer, 2, 301-310.

173. Gloire, G., Charlier, E., Rahmouni, S., Volanti, C., Chariot, A., Erneux, C., \& Piette, J. (2006). Restoration of SHIP-1 activity in human leukemic cells modifies NF-kappaB activation pathway and cellular survival upon oxidative stress. Oncogene, 25, 54855494.

174. Storz, P., Döppler, H., \& Toker, A. (2004). Protein kinase C $\delta$ selectively regulates protein kinase D-dependent activation of $\mathrm{NF}-\mathrm{kB}$ in oxidative stress signaling. Molecular and Cellular Biology, 24, 2614-2626.

175. Storz, P., \& Toker, A. (2003). Protein kinase D mediates a stressinduced NF-kappaB activation and survival pathway. EMBO Journal, 22, 109-120.

176. Fan, C., Li, Q., Ross, D., \& Engelhardt, J. F. (2003). Tyrosine phosphorylation of $\mathrm{I} \kappa \mathrm{B} \alpha$ activates $\mathrm{NF} \kappa \mathrm{B}$ through a redoxregulated and c-Src-dependent mechanism following hypoxia/reoxygenation. Journal of Biological Chemistry, 278, 2072-2080.

177. Zhao, Q., \& Lee, F. S. (1999). Mitogen-activated protein kinase/ ERK kinase kinases 2 and 3 activate nuclear factor- $\mathrm{KB}$ through I $\mathrm{B}$ kinase- $\alpha$ and IKB kinase- $\beta$. Journal of Biological Chemistry, 274, 8355-8358.

178. Li, Q., \& Engelhardt, J. F. (2006). Interleukin-1 $\beta$ induction of $\mathrm{NF} K \mathrm{~B}$ is partially regulated by $\mathrm{H}_{2} \mathrm{O}_{2}$-mediated activation of NFkB-inducing kinase. Journal of Biological Chemistry, 281, 1495-1505.

179. Herscovitch, M., Comb, W., Ennis, T., Coleman, K., Yong, S., Armstead, B., Kalaitzidis, D., Chandani, S., \& Gilmore, T. D. (2008). Intermolecular disulfide bond formation in the NEMO dimer requires Cys54 and Cys347. Biochemical and Biophysical Research Communications, 367, 103-108.
180. Nakajima, S., \& Kitamura, M. (2013). Bidirectional regulation of NF- $\mathrm{kB}$ by reactive oxygen species: a role of unfolded protein response. Free Radical Biological Medicine, 65, 162-174.

181. Scholz, C. C., \& Taylor, C. T. (2013). Hydroxylase-dependent regulation of the NF-kB pathway. Biological Chemistry, 394, 479.

182. Cummins, E. P., Berra, E., Comerford, K. M., Ginouves, A., Fitzgerald, K. T., Seeballuck, F., Godson, C., Nielsen, J. E., Moynagh, P., Pouyssegur, J., \& Taylor, C. T. (2006). Prolyl hydroxylase- 1 negatively regulates IKB kinase- $\beta$, giving insight into hypoxia-induced NFKB activity. Proceedings of National Academy of Sciences U S A, 103, 18154-18159.

183. Cummins, E. P., Comerford, K. M., Scholz, C., Bruning, U., Taylor, C. T., Helmut, S., \& Bernhard, B. (2007). Hypoxic regulation of NF-kappaB signaling. Methods in Enzymology, 435, 479-492.

184. Chu, W. M. (2012). Tumor necrosis factor. Cancer Letters, 328, 222-225.

185. Han, D., Ybanez, M. D., Ahmadi, S., Yeh, K., \& K, N. (2009). Redox regulation of tumor necrosis factor signaling. Antioxidants and Redox Signaling, 11, 2245-2263.

186. Klatt, P., \& Lamas, S. (2000). Regulation of protein function by Sglutathiolation in response to oxidative and nitrosative stress. European Journal of Biochemistry, 267, 4928-4944.

187. Nishi, T., Shimizu, N., Hiramoto, M., Sato, I., Yamaguchi, Y., Hasegawa, M., Aizawa, S., Tanaka, H., Kataoka, K., Watanabe, H., \& Handa, H. (2002). Spatial redox regulation of a critical cysteine residue of NF- $\mathrm{KB}$ in vivo. Journal of Biological Chemistry, 277, 44548-44556.

188. Korn, S. H., Wouters, E. F. M., Vos, N., \& Janssen-Heininger, Y. M. W. (2001). Cytokine-induced activation of nuclear factor- $\mathrm{kB}$ is inhibited by hydrogen peroxide through oxidative inactivation of IкB kinase. Journal of Biological Chemistry, 276, 35693-35700.

189. Sakon, S., Xue, X., Takekawa, M., Sasazuki, T., Okazaki, T., Kojima, Y., Piao, J. H., Yagita, H., Okumura, K., Doi, T., \& Nakano, H. (2003). NF-kB inhibits TNF-induced accumulation of ROS that mediate prolonged MAPK activation and necrotic cell death. EMBO Journal, 22, 3898-3909.

190. Heger, M., Beek, J. F., Moldovan, N. I., van der Horst, C. M. A. M., \& van Gemert, M. J. C. (2005). Towards optimization of selective photothermolysis: prothrombotic pharmaceutical agents as potential adjuvants in laser treatment of port wine stains. A theoretical study. Thrombosis and Haemostasis, 93, 242-256.

191. Guttridge, D. C., Albanese, C., Reuther, J. Y., Pestell, R. G., \& Baldwin, A. S., Jr. (1999). NF-kappa B controls cell growth and differentiation through transcriptional regulation of cyclin D1. Molecular and Cellular Biology, 19, 5785-5799.

192. Hayden, M. S., \& Ghosh, S. (2008). Shared principles in NF-kB signaling. Cell, 132, 344-362.

193. Hinz, M., Krappmann, D., Eichten, A., Heder, A., Scheidereit, C., \& Strauss, M. (1999). NF-kappa B function in growth control: regulation of cyclin D1 expression and G0/G1-to-S-phase transition. Molecular and Cellular Biology, 19, 2690-2698.

194. Buytaert, E., Dewaele, M., \& Agostinis, P. (2007). Molecular effectors of multiple cell death pathways initiated by photodynamic therapy. Biochimica et Biophysica Acta, 1776, 86-107.

195. Volanti, C., Gloire, G., Vanderplasschen, A., Jacobs, N., Habraken, Y., \& Piette, J. (2004). Downregulation of ICAM-1 and VCAM-1 expression in endothelial cells treated by photodynamic therapy. Oncogene, 23, 8649-8658.

196. Karin, M., \& Lin, A. (2002). NF-kappa B at the crossroads of life and death. Nature Immunology, 3, 221-227.

197. van Uden, P., Kenneth, N. S., \& Rocha, S. (2008). Regulation of hypoxia-inducible factor- $1 \alpha$ by NF-kB. Biochemistry Journal, $412,477-484$. 
198. Hymowitz, S. G., \& Wertz, I. E. (2010). A20: from ubiquitin editing to tumour suppression. Nature Reviews Cancer, 10, 332 341.

199. Castano, A. P., Mroz, P., \& Hamblin, M. R. (2006). Photodynamic therapy and anti-tumour immunity. Nature Reviews Cancer, 6, $535-545$.

200. de Groot, D. J. A., de Vries, E. G. E., Groen, H. J. M., \& de Jong, S. (2007). Non-steroidal anti-inflammatory drugs to potentiate chemotherapy effects: from lab to clinic. Critical Reviews in Oncology/Hematology, 61, 52-69.

201. Kaidi, A., Qualtrough, D., Williams, A. C., \& Paraskeva, C. (2006). Direct transcriptional up-regulation of cyclooxygenase-2 by hypoxia-inducible factor (HIF)-1 promotes colorectal tumor cell survival and enhances HIF-1 transcriptional activity during hypoxia. Cancer Research, 66, 6683-6691.

202. Luna, M., Wong, S., Ferrario, A., \& Gomer, C. J. (2008). Cyclooxygenase- 2 expression induced by photofrin photodynamic therapy involves the $\mathrm{p} 38$ MAPK pathway. Photochemistry and Photobiology, 84, 509-514.

203. Tanabe, T., \& Tohnai, N. (2002). Cyclooxygenase isozymes and their gene structures and expression. Prostaglandins Other Lipid Medicine, 68-69, 95-114.

204. Wang, D., \& DuBois, R. N. (2010). Eicosanoids and cancer. Nature Reviews Cancer, 10, 181-193.

205. Buchanan, F. G., Gorden, D. L., Matta, P., Shi, Q., Matrisian, L. M., \& DuBois, R. N. (2006). Role of $\beta$-arrestin 1 in the metastatic progression of colorectal cancer. Proceedings of the National Academy of Sciences of the United States of America, 103, 1492-1497.

206. Buchanan, F. G., Wang, D., Bargiacchi, F., \& DuBois, R. N. (2003). Prostaglandin E2 regulates cell migration via the intracellular activation of the epidermal growth factor receptor. Journal of Biological Chemistry, 278, 35451-35457.

207. Wang, D. \& DuBois, R.N. (2010) Eicosanoids and cancer. National Review Cancer 10:181-193.

208. Han, C., Michalopoulos, G. K., \& Wu, T. (2006). Prostaglandin E2 receptor EP1 transactivates EGFR/MET receptor tyrosine kinases and enhances invasiveness in human hepatocellular carcinoma cells. Journal of Cellular Physiology, 207, 261-270.

209. Ito, H., Duxbury, M., Benoit, E., Clancy, T. E., Zinner, M. J., Ashley, S. W., \& Whang, E. E. (2004). Prostaglandin E2 enhances pancreatic cancer invasiveness through an Ets-1-dependent induction of matrix metalloproteinase-2. Cancer Research, 64, 74397446.

210. Pan, M. R., Hou, M. F., Chang, H. C., \& Hung, W. C. (2008). Cyclooxygenase-2 up-regulates CCR7 via EP2/EP4 receptor signaling pathways to enhance lymphatic invasion of breast cancer cells. Journal of Biological Chemistry, 283, 11155-11163.

211. Abdel-Majid, R. M., \& Marshall, J. S. (2004). Prostaglandin E2 induces degranulation-independent production of vascular endothelial growth factor by human mast cells. Journal of Immunology, $172,1227-1236$

212. Dormond, O., Bezzi, M., Mariotti, A., \& RÃ̄1/4egg, C. (2002). Prostaglandin E2 promotes integrin $\alpha \mathrm{V} \beta 3$-dependent endothelial cell adhesion, Rac-activation, and spreading through cAMP/PKAdependent signaling. Journal of Biological Chemistry, 277, 45838-45846.

213. Salcedo, R., Zhang, X., Young, H. A., Michael, N., Wasserman, K., Ma, W.-H., Martins-Green, M., Murphy, W. J., \& Oppenheim, J. J. (2003). Angiogenic effects of prostaglandin E2 are mediated by up-regulation of CXCR4 on human microvascular endothelial cells. Blood, 102, 1966-1977.

214. Cheung, C. H., Huang, C. C., Tsai, F. Y., Lee, J. Y., Cheng, S. M., Chang, Y. C., Huang, Y. C., Chen, S. H., \& JY, C. (2013). Survivin - biology and potential as a therapeutic target in oncology. Oncology Targets Thermometer, 6, 1453-1462.
215. Boidot, R., Végran, F., \& Lizard-Nacol, S. (2014). Transcriptional regulation of the survivin gene. Molecular Biology Reports, 41 , 233-240.

216. Marusawa, H., Matsuzawa, S., Welsh, K., Zou, H., Armstrong, R., Tamm, I., \& Reed, J. C. (2003). HBXIP functions as a cofactor of survivin in apoptosis suppression. EMBO Journal, 22, 27292740.

217. Dohi, T., Beltrami, E., Wall, N. R., Plescia, J., \& Altieri, D. C. (2004). Mitochondrial survivin inhibits apoptosis and promotes tumorigenesis. Journal of Clinical Investigation, 114, 1117-1127.

218. Wang, H. W., Sharp, T. V., Koumi, A., Koentges, G., \& Boshoff, C. (2002). Characterization of an anti-apoptotic glycoprotein encoded by Kaposis sarcoma-associated herpesvirus which resembles a spliced variant of human survivin. EMBO Journal, 21, 2602-2615.

219. Wang, Z., Jiang, H., Chen, S., Du, F.\& Wang. X. (2012) The mitochondrial phosphatase PGAM5 functions at the convergence point of multiple necrotic death pathways. Cell 148:228-243.

220. de Almagro, M. C., \& Vucic, D. (2012). The inhibitor of apoptosis (IAP) proteins are critical regulators of signaling pathways and targets for anti-cancer therapy. Experimental Oncology, 34, 200 211.

221. Dubrez, L., Berthelet, J., \& Glorian, V. (2013). IAP proteins as targets for drug development in oncology. Oncology Targeted Therapy, 9, 1285-1304.

222. Vallabhapurapu, S., Matsuzawa, A., Zhang, W., Tseng, P.-H., Keats, J. J., Wang, H., Vignali, D. A. A., Bergsagel, P. L., \& Karin, M. (2008). Nonredundant and complementary functions of TRAF2 and TRAF3 in a ubiquitination cascade that activates NIK-dependent alternative NF-[kappa]B signaling. Nature Immunology, 9, 1364-1370.

223. Varfolomeev, E., Goncharov, T., Maecker, H., Zobel, K., Komuves, L. G., Deshayes, K., \& Vucic, D. (2012). Cellular inhibitors of apoptosis are global regulators of NF- $\mathrm{KB}$ and MAPK activation by members of the TNF family of receptors. Science Signaling, 5, ra22.

224. Tanabe, O., Akira, S., Kamiya, T., Wong, G. G., Hirano, T., \& Kishimoto, T. (1988). Genomic structure of the murine IL-6 gene. High degree conservation of potential regulatory sequences between mouse and human. Journal of Immunology, 141, 38753881.

225. Xu, S., \& Neamati, N. (2013). Gp130: a promising drug target for cancer therapy. Expert Opinion on Therapeutic Targets, 17, 13031328.

226. Guo, Y., Xu, F., Lu, T., Duan, Z., \& Zhang, Z. (2012). Interleukin6 signaling pathway in targeted therapy for cancer. Cancer Treatment Reviews, 38, 904-910.

227. Rokavec, M., Öner, M. G., Li, H., Jackstadt, R., Jiang, L., Lodygin, D., Kaller, M., Horst, D., Ziegler, P. K., Schwitalla, S., Slotta-Huspenina, J., Bader, F. G., Greten, F. R., \& Hermeking, H. (2014). IL-6R/STAT3/miR-34a feedback loop promotes EMTmediated colorectal cancer invasion and metastasis. Journal of Clinical Investigation, 124, 1853-1867.

228. Sullivan, N. J., Sasser, A. K., Axel, A. E., Vesuna, F., Raman, V., Ramirez, N., Oberyszyn, T. M., \& Hall, B. M. (2009). Interleukin6 induces an epithelial-mesenchymal transition phenotype in human breast cancer cells. Oncogene, 28, 2940-2947.

229. Yadav, A., Kumar, B., Datta, J., Teknos, T. N., \& Kumar, P. (2011). IL-6 promotes head and neck tumor metastasis by inducing epithelial-mesenchymal transition via the JAK-STAT3-SNAIL signaling pathway. Molecular Cancer Research, 9, 1658-1667.

230. Yu, H., Pardoll, D., \& Jove, R. (2009). STATs in cancer inflammation and immunity: a leading role for STAT3. Nature Reviews Cancer, 9, 798-809. 
231. Grivennikov, S. I., \& Karin, M. (2010). Dangerous liaisons: STAT3 and NF- $\mathrm{kB}$ collaboration and crosstalk in cancer. Cytokine and Growth Factor Reviews, 21, 11-19.

232. Overall, C. M., \& Kleifeld, O. (2006). Validating matrix metalloproteinases as drug targets and anti-targets for cancer therapy. Nature Reviews Cancer, 6, 227-239.

233. Kessenbrock, K., Plaks, V., \& Werb, Z. (2010). Matrix metalloproteinases: regulators of the tumor microenvironment. Cell, 141, 52-67.

234. Bergers, G., Javaherian, K., Lo, K.-M., Folkman, J., \& Hanahan, D. (1999). Effects of angiogenesis inhibitors on multistage carcinogenesis in mice. Science, 284, 808-812.

235. Granville, D. J., Carthy, C. M., Jiang, H., Levy, J. G., McManus, B. M., Matroule, J. Y., Piette, J., \& Hunt, D. W. C. (2000). Nuclear factor-kappa B activation by the photochemotherapeutic agent verteporfin. Blood, 95, 256-262.

236. Korbelik, M. (2009). Complement upregulation in photodynamic therapy-treated tumors: role of Toll-like receptor pathway and NFkappaB. Cancer Letters, 281, 232-238.

237. Matroule, J. Y., Hellin, A. C., Morliere, P., Fabiano, A. S., Santus, R., Merville, M. P., \& Piette, J. (1999). Role of nuclear factor$\mathrm{KB}$ in colon cancer cell apoptosis mediated by aminopyropheophorbide photosensitization. Photochemistry and Photobiology, 70, 540-548.

238. Ryter, S. W. G. C. (1993). Nuclear factor kappa B binding activity in mouse L1210 cells following photofrin II-mediated photosensitization. Photochemistry and Photobiology, 58, 753-756.

239. Volanti, C., Hendrickx, N., Van Lint, J., Matroule, J.-Y., Agostinis, P., \& Piette, J. (2005). Distinct transduction mechanisms of cyclooxygenase 2 gene activation in tumour cells after photodynamic therapy. Oncogene, 24, 2981-2991.

240. Du, H., Olivo, M., Mahendran, R., Huang, Q., Shen, H., Ong, C., \& Bay, B. (2007). Hypericin photoactivation triggers downregulation of matrix metalloproteinase-9 expression in welldifferentiated human nasopharyngeal cancer cells. Cellular and Molecular Life Sciences, 64, 979-988.

241. Li, B., Chu, X. M., Gao, M. H., \& Li, W. X. (2010). Apoptotic mechanism of MCF-7 breast cells in vivo and in vitro induced by photodynamic therapy with C-phycocyanin. Acta Biochimica et Biophysica Sinica, 42, 80-89.

242. Ferrario, A., von Tiehl, K., Wong, S., Luna, M., \& Gomer, C. J. (2002). Cyclooxygenase-2 inhibitor treatment enhances photodynamic therapy-mediated tumor response. Cancer Research, 62, 3956-3961.

243. Hendrickx, N., Dewaele, M., Buytaert, E., Marsboom, G., Janssens, S., Boven, M. V., Vandenheede, J. R., de Witte, P., \& Agostinis, P. (2005). Targeted inhibition of p38 $\alpha$ MAPK suppresses tumor-associated endothelial cell migration in response to hypericin-based photodynamic therapy. Biochemical and Biophysical Research Communications, 337, 928-935.

244. Hendrickx, N., Volanti, C., Moens, U., Seternes, O. M., de Witte, P., Vandenheede, J. R., Piette, J., \& Agostinis, P. (2003). Upregulation of cyclooxygenase- 2 and apoptosis resistance by $\mathrm{p} 38$ MAPK in hypericin-mediated photodynamic therapy of human cancer cells. Journal of Biological Chemistry, 278, 52231-52239.

245. Makowski, M., Grzela, T., Niderla, J., ŁAzarczyk, M., Mróz, P., Kopeé, M., Legat, M., Strusińska, K., Koziak, K., Nowis, D., Mrówka, P., Wasik, M., Jakóbisiak, M., \& Golab, J. (2003). Inhibition of cyclooxygenase- 2 indirectly potentiates antitumor effects of photodynamic therapy in mice. Clinical Cancer Research, 9, 5417-5422.

246. Zhou, Q., Olivo, M., Lye, K., Moore, S., Sharma, A., \& Chowbay, B. (2005). Enhancing the therapeutic responsiveness of photodynamic therapy with the antiangiogenic agents SU5416 and SU6668 in murine nasopharyngeal carcinoma models. Cancer Chemotherapy and Pharmacology, 56, 569-577.
247. Jee, S. H., Shen, S. C., Chiu, H. C., Tsai, W. L., \& Kuo, M. L. (2001). Overexpression of interleukin-6 in human basal cell carcinoma cell lines increases anti-apoptotic activity and tumorigenic potency. Oncogene, 20, 198.

248. Gomer, C. J., Luna, M., Ferrario, A., Wong, S., \& Gomer, C. (2010). Identification of MAP kinase pathways involved in COX-2 expression following photofrin photodynamic therapy. Methods in Molecular Biology, 635, 47-63.

249. Cogno, I. S., Vittar, N. B. R., Lamberti, M. J., \& Rivarola, V. A. (2011). Optimization of photodynamic therapy response by survivin gene knockdown in human metastatic breast cancer T47D cells. Journal of Photochemistry and Photobiology B, 104, 434-443.

250. Ferrario, A., \& Gomer, C. J. (2010). Targeting the $90 \mathrm{kDa}$ heat shock protein improves photodynamic therapy. Cancer Letters, 289, 188-194.

251. Ferrario, A., Lim, S., Xu, F., Luna, M., Gaffney, K. J., Petasis, N. A., Schönthal, A. H., \& Gomer, C. J. (2011). Enhancement of photodynamic therapy by 2,5-dimethyl celecoxib, a noncyclooxygenase-2 inhibitor analog of celecoxib. Cancer Letters, 304, 33-40.

252. Ferrario, A., Rucker, N., Wong, S., Luna, M., \& Gomer, C. J. (2007). Survivin, a member of the inhibitor of apoptosis family, is induced by photodynamic therapy and is a target for improving treatment response. Cancer Research, 67, 4989-4995.

253. He, G. F., Bian, M. L., Zhao, Y. W., Xiang, Q., Li, H. Y., \& Xiao, C. (2009). A study on the mechanism of 5-aminolevulinic acid photodynamic therapy in vitro and in vivo in cervical cancer. Oncology Reports, 21, 861-868.

254. Zhang, M., Yang, J., \& Li, F. (2006). Transcriptional and posttranscriptional controls of survivin in cancer cells: novel approaches for cancer treatment. Journal of Experimental \& Clinical Cancer Research : CR, 25, 391-402.

255. Du, H., Bay, B.-H., Mahendran, R., \& Olivo, M. (2006). Hypericin-mediated photodynamic therapy elicits differential interleukin-6 response in nasopharyngeal cancer. Cancer Letters, 235, 202-208.

256. Brackett, C. M., Owczarczak, B., Ramsey, K., Maier, P. G., \& Gollnick, S. O. (2011). IL-6 potentiates tumor resistance to photodynamic therapy (PDT). Lasers in Surgery and Medicine, 43, 676-685.

257. Wang, Q., Horiatis, D., \& Pinski, J. (2004). Interleukin-6 inhibits the growth of prostate cancer xenografts in mice by the process of neuroendocrine differentiation. International Journal of Cancer, $111,508-513$.

258. Hatzi, E., Murphy, C., Zoephel, A., Rasmussen, H., Morbidelli, L., Ahorn, H., Kunisada, K., Tontsch, U., Klenk, M., YamauchiTakihara, K., Ziche, M., Rofstad, E. K., Schweigerer, L., \& Fotsis, L. (2002). N-myc oncogene overexpression downregulates IL-6; evidence that IL-6 inhibits angiogenesis and suppresses neuroblastoma tumor growth. Oncogene, 21, 3552-3561.

259. Usuda, J., Okunaka, T., Furukawa, K., Tsuchida, T., Kuroiwa, Y., Ohe, Y., Saijo, N., Nishio, K., Konaka, C., \& Kato, H. (2001). Increased cytotoxic effects of photodynamic therapy in IL-6 gene transfected cells via enhanced apoptosis. International Journal of Cancer, 93, 475-480.

260. Cheon, Y. K., Cho, Y. D., Moon, J. H., Jang, J. Y., Kim, Y. S., Kim, Y. S., Lee, M. S., Lee, J. S., \& Shim, C. S. (2007). Diagnostic utility of interleukin-6 (IL-6) for primary bile duct cancer and changes in serum IL-6 levels following photodynamic therapy. American Journal of Gastroenterology, 102, 2164-2170.

261. Agelopoulos, M., \& Thanos, D. (2006). Epigenetic determination of a cell-specific gene expression program by ATF-2 and the histone variant macroH2A. EMBO Journal, 25, 4843-4853.

262. Eferl, R., \& Wagner, E. F. (2003). AP-1: a double-edged sword in tumorigenesis. Nature Reviews Cancer, 3, 859-868. 
263. Bond, M., Fabunmi, R. P., Baker, A. H., \& Newby, A. C. (1998). Synergistic upregulation of metalloproteinase- 9 by growth factors and inflammatory cytokines: an absolute requirement for transcription factor NF-kB. FEBS Letters, 435, 29-34.

264. Filip, A., Clichici, S., Muresan, A., Daicoviciu, D., Tatomir, C., Login, C., Dreve, S., \& Gherman, C. (2008). Effects of PDT with 5 -aminolevulinic acid and chitosan on Walker carcinosarcoma. Experimental Oncology, 30, 212-219.

265. Karrer, S., Bosserhoff, A. K., Weiderer, P., Landthaler, M., \& Szeimies, R. M. (2004). Keratinocyte-derived cytokines after photodynamic therapy and their paracrine induction of matrix metalloproteinases in fibroblasts. British Journal of Dermatology, 151, 776-783.

266. Osiecka, B., Jurczyszyn, K., Symonowicz, K., Bronowicz, A., Ostasiewicz, P., Czapińska, E., Hotowy, K., Krzystek-Korpacka, M., Gebarowska, E., Izykowska, I., Dziegiel, P., Terlecki, G., \& Ziółkowski, P. (2010). In vitro and in vivo matrix metalloproteinase expression after photodynamic therapy with a liposomal formulation of aminolevulinic acid and its methyl ester. Cellular \& Molecular Biology Letters, 15, 630-650.

267. Almeida Issa, M. C., Piñeiro-Maceira, J., Farias, R. E., Pureza, M., Raggio Luiz, R., \& Manela-Azulay, M. (2009). Immunohistochemical expression of matrix metalloproteinases in photodamaged skin by photodynamic therapy. British Journal of Dermatology, 161, 647-653.

268. Au, C. M., Luk, S. K., Jackson, C. J., Ng, H. K., Yow, C. M. N., \& To, S. S. T. (2006). Differential effects of photofrin, 5aminolevulinic acid and calphostin $\mathrm{C}$ on glioma cells. Journal of Photochemistry and Photobiology B, 85, 92-101

269. Chu, E. S., Wong, T. K., \& Yow, C. M. (2008). Photodynamic effect in medulloblastoma: downregulation of matrix metalloproteinases and human telomerase reverse transcriptase expressions. Photochemistry \& Photobiological Sciences, 7, 76-83.

270. Sharwani, A., Jerjes, W., Hopper, C., Lewis, M. P., El-Maaytah, M., Khalil, H. S. M., MacRobert, A. J., Upile, T., \& Salih, V. (2006). Photodynamic therapy down-regulates the invasion promoting factors in human oral cancer. Archives of Oral Biology, 51, 1104-1111.

271. Etminan, N., Peters, C., Ficnar, J., Anlasik, S., Bünemann, E., Slotty, P. J., Hänggi, D., Steiger, H.-J., Sorg, R. V., \& Stummer, W. (2011). Modulation of migratory activity and invasiveness of human glioma spheroids following 5-aminolevulinic acid-based photodynamic treatment. Journal of Neurosurgery, 115, 281-288.

272. Salminen, A., Lehtonen, M., Suuronen, T., Kaarniranta, K., \& Huuskonen, J. (2008). Terpenoids: natural inhibitors of NF-kB signaling with anti-inflammatory and anticancer potential. Cellular and Molecular Life Sciences, 65, 2979-2999.

273. Coupienne, I., Sb, B., Dewaele, M., Rubio, N., Habraken, Y., Fulda, S., Agostinis, P., \& Piette, J. (2011). NF-kappaB inhibition improves the sensitivity of human glioblastoma cells to 5aminolevulinic acid-based photodynamic therapy. Biochemical Pharmacology, 81, 606-616.

274. Broekgaarden, M., Kos, M., Jurg, F. A., Van Beek, A. A., Van Gulik, T. M., \& Heger, M. (2015). Inhibition of NF-kB in tumor cells exacerbates immune cell activation following photodynamic therapy. International Journal of Molecular Sciences, 16, 1996019977.

275. Chen, H. M., Liu, C. M., Yang, H., Chou, H. Y., Chiang, C. P., \& Kuo, M. Y. (2011). 5-Aminolevulinic acid induce apoptosis via $\mathrm{NF}-\mathrm{kB} / \mathrm{JNK}$ pathway in human oral cancer Ca9-22 cells. Journal of Oral Pathology \& Medicine, 40, 483-489.

276. Rapozzi, V., Umezawa, K., \& Xodo, L. E. (2011). Role of NF-kB/ Snail/RKIP loop in the response of tumor cells to photodynamic therapy. Lasers in Surgery and Medicine, 43, 575-585.

277. Tennant, D. A., Frezza, C., MacKenzie, E. D., Nguyen, Q. D., Zheng, L., Selak, M. A., Roberts, D. L., Dive, C., Watson, D.
G., Aboagye, E. O., \& Gottlieb, E. (2009). Reactivating HIF prolyl hydroxylases under hypoxia results in metabolic catastrophe and cell death. Oncogene, 28, 4009-4021.

278. Gomer, C. J., Ferrario, A., Luna, M., Rucker, N., \& Wong, S. (2006). Photodynamic therapy: combined modality approaches targeting the tumor microenvironment. Lasers in Surgery and Medicine, 38, 516-521.

279. Akita, Y., Kozaki, K., Nakagawa, A., Saito, T., Ito, S., Tamada, Y., Fujiwara, S., Nishikawa, N., Uchida, K., Yoshikawa, K., Noguchi, T., Miyaishi, O., Shimozato, K., Saga, S., \& Matsumoto, Y. (2004). Cyclooxygenase-2 is a possible target of treatment approach in conjunction with photodynamic therapy for various disorders in skin and oral cavity. British Journal of Dermatology, $151,472-480$.

280. Ferrario, A., Fisher, A. M., Rucker, N., \& Gomer, C. J. (2005). Celecoxib and NS-398 enhance photodynamic therapy by increasing in vitro apoptosis and decreasing in vivo inflammatory and angiogenic factors. Cancer Research, 65, 9473-9478.

281. Harvey, E. H., Webber, J., Kessel, D., \& Fromm, D. (2005). Killing tumor cells: the effect of photodynamic therapy using mono-l-aspartyl chlorine and NS-398. American Journal of Surgery, 189, 302-305.

282. Lanzo, C. A., Beechem, J. M., Talley, J., \& Marnett, L. J. (1998). Investigation of the binding of isoform-selective inhibitors to prostaglandin endoperoxide synthases using fluorescence spectroscopy. Biochemistry, 37, 217-226.

283. Luong, C., Miller, A., Barnett, J., Chow, J., Ramesha, C., \& B, M. F. (1996). Flexibility of the NSAID binding site in the structure of human cyclooxygenase-2. Natural Structural Biology, 3, 927933.

284. Ulrich, C. M., Bigler, J., \& Potter, J. D. (2006). Nonsteroidal anti-inflammatory drugs for cancer prevention: promise, perils and pharmacogenetics. Nature Reviews Cancer, 6, 130-140.

285. Futaki, N., Takahashi, S., Yokoyama, M., Arai, I., Higuchi, S., \& Otomo, S. (1994). NS-398, a new anti-inflammatory agent, selectively inhibits prostaglandin $\mathrm{G} / \mathrm{H}$ synthase/cyclooxygenase (COX-2) activity in vitro. Prostaglandins, 47, 55-59.

286. Kleban, J., Mikeš, J., Szilárdiová, B., Koval', J., Sačková, V., Solár, P., Horváth, V., Hofmanová, J., Kozubík, A., \& Fedoročko, P. (2007). Modulation of hypericin photodynamic therapy by pretreatment with 12 various inhibitors of arachidonic acid metabolism in colon adenocarcinoma HT-29 cells. Photochemistry and Photobiology, 83, 1174-1185.

287. Chang, C. C., Heller, J. D., Kuo, J., \& Huang, R. C. C. (2004). Tetra-O-methyl nordihydroguaiaretic acid induces growth arrest and cellular apoptosis by inhibiting $\mathrm{Cdc} 2$ and survivin expression. Proceedings of the National Academy of Sciences of the United States of America, 101, 13239-13244.

288. Scheller, J., Garbers, C., \& Rose-John, S. (2014). Interleukin-6: from basic biology to selective blockade of pro-inflammatory activities. Seminars in Immunology, 26, 2-12.

289. Ferrario, A., Chantrain, C. F., von Tiehl, K., Buckley, S., Rucker, N., Shalinsky, D. R., Shimada, H., DeClerck, Y. A., \& Gomer, C. J. (2004). The matrix metalloproteinase inhibitor prinomastat enhances photodynamic therapy responsiveness in a mouse tumor model. Cancer Research, 64, 2328-2332.

290. Semenza, G. L. (2007). Evaluation of HIF-1 inhibitors as anticancer agents. Drug Discovery Today, 12, 853-859.

291. Ferrario, A., von Tiehl, K. F., Rucker, N., Schwarz, M. A., Gill, P. S., \& Gomer, C. J. (2000). Antiangiogenic treatment enhances photodynamic therapy responsiveness in a mouse mammary carcinoma. Cancer Research, 60, 4066-4069.

292. Krieg, R. C., Raupach, K., Ren, Q., Schwamborn, K., \& Knuechel, R. (2007). Analyzing effects of photodynamic therapy with 5-aminolevulinic acid (ALA) induced protoporphyrin IX 
(PPIX) in urothelial cells using reverse phase protein arrays. Photochemistry \& Photobiological Sciences, 6, 1296-1305.

293. Mitra, S., Cassar, S. E., Niles, D. J., Puskas, J. A., Frelinger, J. G., \& Foster, T. H. (2006). Photodynamic therapy mediates the oxygen-independent activation of hypoxia-inducible factor $1 \alpha$. Molecular Cancer Therapeutics, 5, 3268-3274.

294. Moon, J. W., Kim, Y. J., Khwarg, S. I., Chung, H., \& Yu, H. G. (2010). Chorioretinal ischemia and angiogenic milieu following photodynamic therapy. Current Eye Research, 35, 314-321.

295. Jiang, B. H., Semenza, G. L., Bauer, C., \& Marti, H. H. (1996). Hypoxia-inducible factor 1 levels vary exponentially over a physiologically relevant range of $\mathrm{O}_{2}$ tension. American Journal of Physiology, 271, C1172-1180.

296. Bracken, C. P., Whitelaw, M. L., \& Peet, D. J. (2003). The hypoxia-inducible factors: key transcriptional regulators of hypoxic responses. Cellular and Molecular Life Sciences, 60, 13761393.

297. Denko, N. C. (2008). Hypoxia, HIF1 and glucose metabolism in the solid tumour. Nature Reviews Cancer, 8, 705-713.

298. Schödel, J., Oikonomopoulos, S., Ragoussis, J., Pugh, C. W., Ratcliffe, P. J., \& Mole, D. R. (2011). High-resolution genomewide mapping of HIF-binding sites by ChIP-seq. Blood, 117, e207-e217.

299. Semenza, G. L. (2012). Hypoxia-inducible factors: mediators of cancer progression and targets for cancer therapy. Trends in Pharmacological Sciences, 33, 207-214.

300. Wu, M. Z., Tsai, Y. P., Yang, M. H., Huang, C. H., Chang, S. Y., Chang, C. C., Teng, S. C., \& Wu, K. J. (2011). Interplay between HDAC3 and WDR5 is essential for hypoxia-induced epithelialmesenchymal transition. Molecular Cell, 43, 811-822.

301. Xia, X., Lemieux, M. E., Li, W., Carroll, J. S., Brown, M., Liu, X. S., \& Kung, A. L. (2009). Integrative analysis of HIF binding and transactivation reveals its role in maintaining histone methylation homeostasis. Proceedings of the National Academy of Sciences of the United States of America, 106, 4260-4265.

302. Bruick, R. K., \& McKnight, S. L. (2001). A conserved family of prolyl-4-hydroxylases that modify HIF. Science, 294, 1337-1340.

303. Epstein, A. C. R., Gleadle, J. M., McNeill, L. A., Hewitson, K. S., O’Rourke, J., Mole, D. R., Mukherji, M., Metzen, E., Wilson, M. I., Dhanda, A., Tian, Y.-M., Masson, N., Hamilton, D. L., Jaakkola, P., Barstead, R., Hodgkin, J., Maxwell, P. H., Pugh, C. W., Schofield, C. J., \& Ratcliffe, P. J. (2001). C. elegans EGL-9 and mammalian homologs define a family of dioxygenases that regulate HIF by prolyl hydroxylation. Cell, 107, 43-54.

304. Ivan, M., Kondo, K., Yang, H., Kim, W., Valiando, J., Ohh, M., Salic, A., Asara, J. M., Lane, W. S., \& Kaelin, W. G., Jr. (2001). HIFalpha targeted for VHL-mediated destruction by proline hydroxylation: implications for $\mathrm{O}_{2}$ sensing. Science, 292, 464-468.

305. Jaakkola, P., Mole, D. R., Tian, Y.-M., Wilson, M. I., Gielbert, J., Gaskell, S. J., Kriegsheim, A., Hebestreit, H. F., Mukherji, M., Schofield, C. J., Maxwell, P. H., Pugh, C. W., \& Ratcliffe, P. J. (2001). Targeting of HIF-alpha to the von Hippel-Lindau ubiquitylation complex by $\mathrm{O}_{2}$-regulated prolyl hydroxylation. Science, 292, 468-472.

306. Maxwell, P. H., Wiesener, M. S., Chang, G.-W., Clifford, S. C., Vaux, E. C., Cockman, M. E., Wykoff, C. C., Pugh, C. W., Maher, E. R., \& Ratcliffe, P. J. (1999). The tumour suppressor protein VHL targets hypoxia-inducible factors for oxygen-dependent proteolysis. Nature, 399, 271-275.

307. Salceda, S., \& Caro, J. (1997). Hypoxia-inducible factor $1 \alpha$ (HIF$1 \alpha$ protein is rapidly degraded by the ubiquitin-proteasome system under normoxic conditions. Journal of Biological Chemistry, 272, 22642-22647.

308. Stiehl, D. P., Wirthner, R., Köditz, J., Spielmann, P., Camenisch, G., \& Wenger, R. H. (2006). Increased prolyl 4-hydroxylase domain proteins compensate for decreased oxygen levels. Journal of Biological Chemistry, 281, 23482-23491.

309. Chandel, N. S., Maltepe, E., Goldwasser, E., Mathieu, C. E., Simon, M. C., \& Schumacker, P. T. (1998). Mitochondrial reactive oxygen species trigger hypoxia-induced transcription. Proceedings of National Academy of Sciences U S A, 95, 11715-11720.

310. Comito, G., Calvani, M., Giannoni, E., Bianchini, F., Calorini, L., Torre, E., Migliore, C., Giordano, S., \& Chiarugi, P. (2011). HIF$1 \alpha$ stabilization by mitochondrial ROS promotes Met-dependent invasive growth and vasculogenic mimicry in melanoma cells. Free Radical Biology Medicine, 51, 893-904.

311. Patten, D. A., Lafleur, V. N., Robitaille, G. A., Chan, D. A., Giaccia, A. J., \& Richard, D. E. (2010). Hypoxia-inducible factor-1 activation in nonhypoxic conditions: the essential role of mitochondrial-derived reactive oxygen species. Molecular Biology of the Cell, 21, 3247-3257.

312. Schofield, C. J., \& Ratcliffe, P. J. (2004). Oxygen sensing by HIF hydroxylases. Nature Reviews Molecular Cell Biology, 5, 343354.

313. Lu, H., Dalgard, C. L., Mohyeldin, A., McFate, T., Tait, A. S., \& Verma, A. (2005). Reversible inactivation of HIF-1 prolyl hydroxylases allows cell metabolism to control basal HIF-1. Journal of Biological Chemistry, 280, 41928-41939.

314. Calvani, M., Comito, G., Giannoni, E., \& Chiarugi, P. (2012). Time-dependent stabilization of hypoxia inducible factor- $1 \alpha$ by different intracellular sources of reactive oxygen species. PloS One, 7, e38388.

315. Bonello, S., Záhringer, C., BelAiba, R. S., Djordjevic, T., Hess, J., Michiels, C., Kietzmann, T., \& Görlach, A. (2007). Reactive oxygen species activate the HIF-1 $\alpha$ promoter via a functional NFKB site. Arteriosclerosis, Thrombosis, and Vascular Biology, 27, 755761.

316. Harris, A. L. (2002). Hypoxia - a key regulatory factor in tumour growth. Nature Reviews Cancer, 2, 38-47.

317. Hirota, K., \& Semenza, G. L. (2006). Regulation of angiogenesis by hypoxia-inducible factor 1. Oncological Hematology, 59, 1526.

318. Weinhouse, S., Warburg, O., Burk, D., \& Schade, A. L. (1956). On respiratory impairment in cancer cells. Science, 124, 267-272.

319. Semenza, G. L. (2003). Targeting HIF-1 for cancer therapy. Nature Reviews Cancer, 3, 721-732.

320. Rohwer, N., \& Cramer, T. (2011). Hypoxia-mediated drug resistance: novel insights on the functional interaction of HIFs and cell death pathways. Drug Resistance Updates, 14, 191-201.

321. Bellot, G., Garcia-Medina, R., Gounon, P., Chiche, J., Roux, D., Pouysségur, J., \& Mazure, N. M. (2009). Hypoxia-induced autophagy is mediated through hypoxia-inducible factor induction of BNIP3 and BNIP3L via their BH3 domains. Molecular and Cellular Biology, 29, 2570-2581.

322. An, W. G., Kanekal, M., Simon, M. C., Maltepe, E., Blagoskonny, M.V., Neckers, L. M. (1998). Stabilization of wild-type p53 by hypoxia-inducible factor $1 \alpha$. Nature, 392, 405-408.

323. Ravi, R., Mookerjee, B., Bhujwalla, Z. M., Sutter, C. H., Artemov, D., Zeng, Q., Dillehay, L. E., Madan, A., Semenza, G. L., Bedi, A. (2000). Regulation of tumor angiogenesis by p53-induced degradation of hypoxia-inducible factor $1 \alpha$. Genes and Devevelopment, 14, 34- 44 .

324. Ji, Z., Yang, G., Shahzidi, S., Tkacz-Stachowska, K., Suo, Z., Nesland, J. M., \& Peng, Q. (2006). Induction of hypoxiainducible factor- $1 \alpha$ overexpression by cobalt chloride enhances cellular resistance to photodynamic therapy. Cancer Letters, 244 , 182-189.

325. Berra, E., Roux, D., Richard, D. E., \& Pouysségur, J. (2001). Hypoxia inducible factor $1 \alpha(\mathrm{HIF}-1 \alpha)$ escapes O2-driven 
proteasomal degradation irrespective of its subcellular localization: nucleus or cytoplasm. EMBO Reports, 2, 615-620.

326. Ferrario, A., \& Gomer, C. (2006). Avastin enhances photodynamic therapy treatment of Kaposi's sarcoma in a mouse tumor model. Journal of Environmental Pathology, Toxicology and Oncology, $25,251-260$.

327. Koukourakis, M. I., Giatromanolaki, A., Skarlatos, J., Corti, L., Blandamura, S., Piazza, M., Gatter, K. C., \& Harris, A. L. (2001). Hypoxia inducible factor (HIF-1a and HIF-2a) expression in early esophageal cancer and response to photodynamic therapy and radiotherapy. Cancer Research, 61, 1830-1832.

328. Mayerhofer, M., Valent, P., Sperr, W. R., Griffin, J. D., \& Sillaber, C. (2002). BCR/ABL induces expression of vascular endothelial growth factor and its transcriptional activator, hypoxia inducible factor- $1 \alpha$, through a pathway involving phosphoinositide 3-kinase and the mammalian target of rapamycin. Blood, 100, 3767-3775.

329. Luwor, R. B., Lu, Y., Li, X., Mendelsohn, J., \& Fan, Z. (2005). The antiepidermal growth factor receptor monoclonal antibody cetuximab/C225 reduces hypoxia-inducible factor-1 alpha, leading to transcriptional inhibition of vascular endothelial growth factor expression. Oncogene, 24, 4433-4441.

330. Majumder, P. K., Febbo, P. G., Bikoff, R., Berger, R., Xue, Q., McMahon, L. M., Manola, J., Brugarolas, J., McDonnell, T. J., Golub, T. R., Loda, M., Lane, H. A., \& Sellers, W. R. (2004). mTOR inhibition reverses Akt-dependent prostate intraepithelial neoplasia through regulation of apoptotic and HIF-1-dependent pathways. Nature Medicine, 10, 594-601.

331. Liu, Y. V., Baek, J. H., Zhang, H., Diez, R., Cole, R. N., \& Semenza, G. L. (2007). RACK1 competes with HSP90 for binding to HIF- $1 \alpha$ and is required for $\mathrm{O}_{2}$-independent and HSP90 inhibitor-induced degradation of HIF-1 $\alpha$. Molecular Cell, 25, 207-217.

332. Kaelin, W. G. J., \& Ratcliffe, P. J. (2008). Oxygen sensing by metazoans: the central role of the HIF hydroxylase pathway. Molecular Cell, 30, 393-402.

333. Tennant, D., \& Gottlieb, E. (2010). HIF prolyl hydroxylase-3 mediates alpha-ketoglutarate-induced apoptosis and tumor suppression. Journal of Molecular Medicine (Berlin), 88, 839-849.

334. Bae, M. K., Kim, S. H., Jeong, J. W., Lee, Y. M., Kim, H. S., Kim, S. R., Yun, I., Bae, S. K., \& Kim, K. W. (2006). Curcumin inhibits hypoxia-induced angiogenesis via down-regulation of HIF-1. Oncology Report, 15, 1557-1562.

335. Ströfer, M., Jelkmann, W., \& Depping, R. (2011). Curcumin decreases survival of Hep3B liver and MCF-7 breast cancer cells: the role of HIF. Strahlentherapie und Onkologie, 187, 393-400.

336. Heger, M., van Golen, R. F., Broekgaarden, M., \& Michel, M. C. (2013). The molecular basis for the pharmacokinetics and pharmacodynamics of curcumin and its metabolites in relation to cancer. Pharmacological Reviews, 66, 222-307.

337. Ahn, J. C., Kang, J. W., Shin, J. I., \& Chung, P. S. (2012). Combination treatment with photodynamic therapy and curcumin induces mitochondria-dependent apoptosis in AMC-HN3 cells. International Journal of Oncology, 41, 2184-2190.

338. Chan, W. H., \& Wu, H. J. (2004). Anti-apoptotic effects of curcumin on photosensitized human epidermal carcinoma A431 cells. Journal of Biological Chemistry, 92, 200-212.

339. Hattori, K., Naguro, I., Runchel, C., \& Ichijo, H. (2009). The roles of ASK family proteins in stress responses and diseases. Cell Communication Signal, 7, 9 .

340. Noguchi, T., Takeda, K., Matsuzawa, A., Saegusa, K., Nakano, H., Gohda, J., Inoue, J., \& Ichijo, H. (2005). Recruitment of tumor necrosis factor receptor-associated factor family proteins to apoptosis signal-regulating kinase 1 signalosome is essential for oxidative stress-induced cell death. Journal of Biological Chemistry, 280, 37033-37040
341. Saitoh, M., Nishitoh, H., Fujii, M., Takeda, K., Tobiume, K., Sawada, Y., Kawabata, M., Miyazono, K., \& Ichijo, H. (1998). Mammalian thioredoxin is a direct inhibitor of apoptosis signalregulating kinase (ASK) 1. EMBO Journal, 17, 2596-2606.

342. Miki, H., \& Funato, Y. (2012). Regulation of intracellular signalling through cysteine oxidation by reactive oxygen species. Journal of Biochemistry, 151, 255-261.

343. Fujino, G., Noguchi, T., Matsuzawa, A., Yamauchi, S., Saitoh, M., Takeda, K., \& Ichijo, H. (2007). Thioredoxin and TRAF family proteins regulate reactive oxygen species-dependent activation of ASK1 through reciprocal modulation of the N-terminal homophilic interaction of ASK1. Molecular and Cellular Biology, 27, 8152-8163.

344. Ichijo, H. (1999). From receptors to stress-activated MAP kinases. Oncogene, 18, 6087-6093.

345. Minden, A., \& Karin, M. (1997). Regulation and function of the JNK subgroup of MAP kinases. Biochimica et Biophysica Acta, 1333, F85-F104.

346. Cuenda, A., \& Rousseau, S. (2007). p38 MAP-kinases pathway regulation, function and role in human diseases. Biochimica et Biophysica Acta, 1773, 1358-1375.

347. Mittelstadt, P. R., Salvador, J. M., Fornace, A. J., \& Ashwell, J. D. (2005). Activating p38 MAPK: new tricks for an old kinase. Cell Cycle, 4, 1189-1192.

348. Goossens, V., Grooten, J., De Vos, K., \& Fiers, W. (1995). Direct evidence for tumor necrosis factor-induced mitochondrial reactive oxygen intermediates and their involvement in cytotoxicity. Proceedings of the National Academy of Sciences of the United States of America, 92, 8115-8119.

349. Kim, Y. S., Morgan, M. J., Choksi, S., \& Liu, Z. G. (2007). TNFinduced activation of the Nox1 NADPH oxidase and its role in the induction of necrotic cell death. Molecular Cell, 26, 675-687.

350. Lin, Y., Choksi, S., Shen, H. M., Yang, Q. F., Hur, G. M., Kim, Y. S., Tran, J. H., Nedospasov, S. A., \& Liu, Z. G. (2004). Tumor necrosis factor-induced nonapoptotic cell death requires receptorinteracting protein-mediated cellular reactive oxygen species accumulation. Journal of Biological Chemistry, 279, 10822-10828.

351. Roulston, A., Reinhard, C., Amiri, P., \& Williams, L. T. (1998). Early activation of c-Jun N-terminal kinase and p38 kinase regulate cell survival in response to tumor necrosis factor $\alpha$. Journal of Biological Chemistry, 273, 10232-10239.

352. Matsuzawa, A., \& Ichijo, H. (2008). Redox control of cell fate by MAP kinase: physiological roles of ASK1-MAP kinase pathway in stress signaling. Biochimica et Biophysica Acta, 1780, 13251336.

353. Gupta, S., Barrett, T., Whitmarsh, A. J., Cavanagh, J., Sluss, H. K., Dérijard, B., \& D, R. J. (1996). Selective interaction of JNK protein kinase isoforms with transcription factors. EMBO Journal, 15, 2660-2670.

354. Wagner, E. F., \& Nebreda, A. R. (2009). Signal integration by JNK and p38 MAPK pathways in cancer development. Nature Reviews Cancer, 9, 537-549.

355. Fuchs, S. Y., Dolan, L., Davis, R. J., \& R, Z. (1996). Phosphorylation-dependent targeting of c-Jun ubiquitination by Jun N-kinase. Oncogene, 13, 1531-1535.

356. Jaeschke, A., Karasarides, M., Ventura, J.-J., Ehrhardt, A., Zhang, C., Flavell, R. A., Shokat, K. M., \& Davis, R. J. (2006). JNK2 is a positive regulator of the cJun transcription factor. Molecular Cell, 23, 899-911.

357. Yu, R., Chen, C., Mo, Y.-Y., Hebbar, V., Owuor, E. D., Tan, T.-H., \& Kong, A. N. T. (2000). Activation of mitogen-activated protein kinase pathways induces antioxidant response element-mediated gene expression via a Nrf2-dependent mechanism. Journal of Biological Chemistry, 275, 39907-39913.

358. Bakiri, L., Lallemand, D., Bossy-Wetzel, E., \& Yaniv, M. (2000). Cell cycle-dependent variations in c-Jun and JunB 
phosphorylation: a role in the control of cyclin D1 expression. EMBO Journal, 19, 2056-2068.

359. Beier, F., Taylor, A. C., \& LuValle, P. (2000). Activating transcription factor 2 is necessary for maximal activity and serum induction of the cyclin A promoter in chondrocytes. Journal of Biological Chemistry, 275, 12948-12953.

360. Katabami, M., Donninger, H., Hommura, F., Leaner, V. D., Kinoshita, I., Chick, J. F. B., \& Birrer, M. J. (2005). Cyclin A is a c-Jun target gene and is necessary for c-Jun-induced anchorageindependent growth in RAT1a cells. Journal of Biological Chemistry, 280, 16728-16738.

361. Lewis, J. S., Vijayanathan, V., Thomas, T. J., Pestell, R. G., Albanese, C., Gallo, M. A., \& Thomas, T. (2005). Activation of cyclin D1 by estradiol and spermine in MCF-7 breast cancer cells: a mechanism involving the $\mathrm{p} 38 \mathrm{MAP}$ kinase and phosphorylation of ATF-2. Oncology Research, 15, 113-128.

362. Ameyar-Zazoua, M., Wisniewska, M. B., Bakiri, L., Wagner, E. F., Yaniv, M., \& Weitzman, J. B. (2005). AP-1 dimers regulate transcription of the $\mathrm{p} 14 / \mathrm{p} 19 \mathrm{ARF}$ tumor suppressor gene. Oncogene, 24, 2298-2306.

363. Schreiber, M., Kolbus, A., Piu, F., Szabowski, A., MühleSteinlein, U., Tian, J., Karin, M., Angel, P., \& Wagner, E. F. (1999). Control of cell cycle progression by c-Jun is p53 dependent. Genes and Development, 13, 607-619.

364. Shaulian, E., \& Karin, M. (2001). AP-1 in cell proliferation and survival. Oncogene, 20, 2390-2400.

365. Weitzman, J. B., Fiette, L., Matsuo, K., \& Yaniv, M. (2000). JunD protects cells from p53-dependent senescence and apoptosis. Molecular Cell, 6, 1109-1119.

366. Maekawa, T., Sano, Y., Shinagawa, T., Rahman, Z., Sakuma, T., Nomura, S., Licht, J. D., \& Ishii, S. (2007). ATF-2 controls transcription of Maspin and GADD45 $\alpha$ genes independently from p53 to suppress mammary tumors. Oncogene, 27, 1045-1054.

367. Vale-Cruz, D. S., Ma, Q., Syme, J., \& LuValle, P. A. (2008). Activating transcription factor-2 affects skeletal growth by modulating $\mathrm{pRb}$ gene expression. Mechanisms of Development, 125, $843-856$

368. Lopez-Bergami, P., Lau, E., \& Ronai, Z. (2010). Emerging roles of ATF2 and the dynamic AP1 network in cancer. Nature Reviews Cancer, 10, 65-76.

369. Maekawa, T., Bernier, F., Sato, M., Nomura, S., Singh, M., Inoue, Y., Tokunaga, T., Imai, H., Yokoyama, M., Reimold, A., Glimcher, L. H., \& Ishii, S. (1999). Mouse ATF-2 null mutants display features of a severe type of meconium aspiration syndrome. Journal of Biological Chemistry, 274, 17813-17819.

370. Song, H., Ki, S. H., Kim, S. G., \& Moon, A. (2006). Activating transcription factor 2 mediates matrix metalloproteinase-2 transcriptional activation induced by $\mathrm{p} 38$ in breast epithelial cells. Cancer Research, 66, 10487-10496.

371. Herr, I., Posovszky, C., Di Marzio, L., Cifone, M. G., Boehler, T., \& Debatin, K. M. (2000). Autoamplification of apoptosis following ligation of CD95-L, TRAIL and TNF-alpha. Oncogene, 19, 4255-4262.

372. Penix, L. A., Sweetser, M. T., Weaver, W. M., Hoeffler, J. P., Kerppola, T. K., \& Wilson, C. B. (1996). The proximal regulatory element of the interferon- $\gamma$ promoter mediates selective expression in T cells. Journal of Biological Chemistry, 271, 31964 31972.

373. Chen, K. D., Hung, J. J., Huang, H. L., Chang, M. D., \& Lai, Y. K. (1997). Rapid induction of the Grp78 gene by cooperative actions of okadaic acid and heat-shock in 9L rat brain tumor cells. European Journal of Biochemistry, 248, 120-129.

374. Wen, A. Y., Sakamoto, K. M., \& Miller, L. S. (2010). The role of the transcription factor CREB in immune function. Journal of Immunology, 185, 6413-6419.
375. Kasibhatla, S., Brunner, T., Genestier, L., Echeverri, F., Mahboubi, A., \& Green, D. R. (1998). DNA damaging agents induce expression of Fas ligand and subsequent apoptosis in T lymphocytes via the activation of NF-kB and AP-1. Molecular Cell, 1, 543-551.

376. Dunn, K. L., Espino, P. S., Drobic, B., He, S., \& Davie, J. R. (2005). The Ras-MAPK signal transduction pathway, cancer and chromatin remodeling. Biochemical Cell Biology, 83, 1-14.

377. Sonenberg, N., \& Gingras, A. C. (1998). The mRNA 5' capbinding protein eIF4E and control of cell growth. Current Opinion in Cell Biology, 10, 268-275.

378. Liu, G., Zhang, Y., Bode, A. M., Ma, W. Y., \& Dong, Z. (2002). Phosphorylation of 4E-BP1 is mediated by the p38/MSK1 pathway in response to UVB irradiation. Journal of Biological Chemistry, 277, 8810-8816.

379. Wiggin, G. R., Soloaga, A., Foster, J. M., Murray-Tait, V., Cohen, P., \& Arthur, J. S. C. (2002). MSK1 and MSK2 are required for the mitogen- and stress-induced phosphorylation of CREB and ATF1 in fibroblasts. Molecular and Cellular Biology, 22, 2871-2881.

380. Kyriakis, J. M., \& Avruch, J. (2001). Mammalian mitogenactivated protein kinase signal transduction pathways activated by stress and inflammation. Physiological Reviews, 81, 807-869.

381. Huot, J., Houle, F., Marceau, F., \& Landry, J. (1997). Oxidative stress-induced actin reorganization mediated by the p38 mitogenactivated protein kinase/heat shock protein 27 pathway in vascular endothelial cells. Circulation Research, 80, 383-392.

382. Moens, U., \& Kostenko, S. (2013). Structure and function of MK5/PRAK: the loner among the mitogen-activated protein kinase-activated protein kinases. Biological Chemistry, 394, 1115.

383. Gaestel, M. (2006). MAPKAP kinases-MKs - two's company, three's a crowd. Nature Reviews Molecular Cell Biology, 7, 120 130.

384. Emerling, B. M., Platanias, L. C., Black, E., Nebreda, A. R., Davis, R. J., \& Chandel, N. S. (2005). Mitochondrial reactive oxygen species activation of $\mathrm{p} 38$ mitogen-activated protein kinase is required for hypoxia signaling. Molecular and Cellular Biology, $25,4853-4862$.

385. Timoshenko, A. V., Chakraborty, C., Wagner, G. F., \& Lala, P. K. (2006). COX-2-mediated stimulation of the lymphangiogenic factor VEGF-C in human breast cancer. British Journal of Cancer, 94, 1154-1163.

386. Rubio, N., Verrax, J., Dewaele, M., Verfaillie, T., Johansen, T., Piette, J., \& Agostinis, P. (2014). p38MAPK-regulated induction of p62 and NBR1 after photodynamic therapy promotes autophagic clearance of ubiquitin aggregates and reduces reactive oxygen species levels by supporting Nrf2-antioxidant signaling. Free Radical Biological Medicine, 67, 292-303.

387. Chang, L., Kamata, H., Solinas, G., Luo, J.-L., Maeda, S., Venuprasad, K., Liu, Y.-C., \& Karin, M. (2006). The E3 ubiquitin ligase itch couples JNK activation to TNF $\alpha$-induced cell death by inducing c-FLIPL turnover. Cell, 124, 601-613.

388. Ventura, J. J., Hübner, A., Zhang, C., Flavell, R. A., Shokat, K. M., \& Davis, R. J. (2006). Chemical genetic analysis of the time course of signal transduction by JNK. Molecular Cell, 21, 701710.

389. Davis, R. J. (2000). Signal transduction by the JNK group of MAP kinases. Cell, 103, 239-252.

390. Inoshita, S., Takeda, K., Hatai, T., Terada, Y., Sano, M., Hata, J., Umezawa, A., \& Ichijo, H. (2002). Phosphorylation and inactivation of myeloid cell leukemia 1 by JNK in response to oxidative stress. Journal of Biological Chemistry, 277, 43730-43734.

391. Lei, K., \& Davis, R. J. (2003). JNK phosphorylation of Bimrelated members of the Bcl2 family induces Bax-dependent apoptosis. Proceedings of the National Academy of Sciences of the United States of America, 100, 2432-2437.

392. Lei, K., Nimnual, A., Zong, W. X., Kennedy, N. J., Flavell, R. A., Thompson, C. B., Bar-Sagi, D., \& Davis, R. J. (2002). The Bax 
subfamily of Bcl2-related proteins is essential for apoptotic signal transduction by c-Jun NH2-terminal kinase. Molecular and Cellular Biology, 22, 4929-4942.

393. Liu, J., \& Lin, A. (2005). Role of JNK activation in apoptosis: a double-edged sword. Cell Research, 15, 36-42.

394. Buschmann, T., Yin, Z., Bhoumik, A., \& Ronai, Z. (2000). Amino-terminal-derived JNK fragment alters expression and activity of c-Jun, ATF2, and p53 and increases $\mathrm{H} 2 \mathrm{O} 2$-induced cell death. Journal of Biological Chemistry, 275, 16590-16596.

395. Chen, Y. R., Wang, X., Templeton, D., Davis, R. J., \& Tan, T. H. (1996). The role of c-Jun N-terminal kinase (JNK) in apoptosis induced by ultraviolet $\mathrm{C}$ and $\gamma$ radiation: duration of JNK activation may determine cell death and proliferation. Journal of Biological Chemistry, 271, 31929-31936.

396. Guo, J. S., Cho, C. H., Wang, W. P., Shen, X. Z., Cheng, C. L., \& Koo, M. W. (2003). Expression and activities of three inducible enzymes in the healing of gastric ulcers in rats. World Journal of Gastroenterology, 9, 1767-1771.

397. Shen, H. M., Lin, Y., Choksi, S., Tran, J., Jin, T., Chang, L., Karin, M., Zhang, J., \& Liu, Z. G. (2004). Essential roles of receptorinteracting protein and TRAF2 in oxidative stress-induced cell death. Molecular and Cellular Biology, 24, 5914-5922.

398. Adler, V., Yin, Z., Fuchs, S. Y., Benezra, M., Rosario, L., Tew, K. D., Pincus, M. R., Sardana, M., Henderson, C. J., Wolf, C. R., Davis, R. J., \& Ronai, Z. (1999). Regulation of JNK signaling by GSTp. EMBO Journal, 18, 1321-1334.

399. Elsby, R., Kitteringham, N. R., Goldring, C. E., Lovatt, C. A., Chamberlain, M., Henderson, C. J., Wolf, C. R., \& Park, B. K. (2003). Increased constitutive c-Jun N-terminal kinase signaling in mice lacking glutathione S-transferase Pi. Journal of Biological Chemistry, 278, 22243-22249.

400. Thévenin, A. F., Zony, C. L., Bahnson, B. J., \& Colman, R. F. (2011). GSTpi modulates JNK activity through a direct interaction with JNK substrate, ATF2. Protein Sciences, 20, 834-848.

401. Wang, T., Arifoglu, P., Ronai, Z., \& Tew, K. D. (2001). Glutathione S-transferase P1-1 (GSTP1-1) inhibits c-Jun N-terminal kinase (JNK1) signaling through interaction with the $\mathrm{C}$ terminus. Journal of Biological Chemistry, 276, 20999-21003.

402. Yin, Z., Ivanov, V. N., Habelhah, H., Tew, K., \& Ronai, Z. (2000). Glutathione S-transferase $p$ elicits protection against $\mathrm{H}_{2} \mathrm{O}_{2}$-induced cell death via coordinated regulation of stress kinases. Cancer Research, 60, 4053-4057.

403. Gupta, M., Gupta, S. K., Hoffman, B., \& Liebermann, D. A. (2006). Gadd45a and Gadd45b protect hematopoietic cells from UV-induced apoptosis via distinct signaling pathways, including p38 activation and JNK inhibition. Journal of Biological Chemistry, 281, 17552-17558.

404. Papa, S., Monti, S. M., Vitale, R. M., Bubici, C., Jayawardena, S., Alvarez, K., De Smaele, E., Dathan, N., Pedone, C., Ruvo, M., \& Franzoso, G. (2007). Insights into the structural basis of the GADD $45 \beta$-mediated Inactivation of the JNK kinase, MKK7/JNKK2. Journal of Biological Chemistry, 282, 19029_ 19041.

405. Papa, S., Bubici, C., Zazzeroni, F., Pham, C. G., Kuntzen, C., Knabb, J. R., Dean, K., \& Franzoso, G. (2006). The NF[kappa]B-mediated control of the JNK cascade in the antagonism of programmed cell death in health and disease. Cell Death and Differentiation, 13, 712-729.

406. Luna, M. C., Wong, S., \& Gomer, C. J. (1994). Photodynamic therapy mediated induction of early response genes. Cancer Research, 54, 1374-1380.

407. Tao, J. S., Sanghera, J. S., Pelech, S. L., Wong, G., \& Levy, J. G. (1996). Stimulation of stress-activated protein kinase and p38 HOG1 kinase in murine keratinocytes following photodynamic therapy with benzoporphyrin derivative. Journal of Biological Chemistry, 271, 27107-27115.
408. Huang, Y., Minigh, J., Miles, S., \& Niles, R. (2008). Retinoic acid decreases ATF-2 phosphorylation and sensitizes melanoma cells to taxol-mediated growth inhibition. Journal of Molecular Signaling, 3, 3 .

409. Fisher, G. J., Talwar, H. S., Lin, J., Lin, P., McPhillips, F., Wang, Z., Li, X., Wan, Y., Kang, S., \& Voorhees, J. J. (1998). Retinoic acid inhibits induction of c-Jun protein by ultraviolet radiation that occurs subsequent to activation of mitogen-activated protein kinase pathways in human skin in vivo. Journal of Clinical Investigation, 101, 1432-1440.

410. Li, J. J., Dong, Z., Dawson, M. I., \& Colburn, N. H. (1996). Inhibition of tumor promoter-induced transformation by retinoids that transrepress AP-1 without transactivating retinoic acid response element. Cancer Research, 56, 483-489.

411. Assefa, Z., Vantieghem, A., Declercq, W., Vandenabeele, P., Vandenheede, J. R., Merlevede, W., de Witte, P., Agostinis, P., The activation of the c-Jun N-terminal kinase and p38 mitogenactivated protein kinase signaling pathways protects HeLa cells from apoptosis following photodynamic therapy with hypericin. Journal of Biological Chemistry, 274, 8788-8796.

412. Xue, L., He, J., Oleinick, N. L. (1999), Promotion of photodynamic therapy-induced apoptosis by stress kinases. Cell Death and Differentiation, 6, 855-864.

413. Tong, Z., Singh, G., Valerie, K., Rainbow, A. J. (2003). Activation of the stress-activated JNK and p38 MAP kinases in human cells by Photofrin-mediated photodynamic therapy. Journal of Photochemistry and Photobiology B, 71, 77-85.

414. Buytaert, E., Matroule, J. Y., Durinck, S., Close, O., Kocanova, S., Vandenheede, J. R., de Witte, P. A., Piette, J., Agostinis, P. (2008). Molecular effectors and modulators of hypericinmediated cell death in bladder cancer cells. Oncogene, 27, 1916-1929.

415. Weyergang, A., Kaalhus, O., Berg, K. (2008). Photodynamic therapy with an endocytically located photosensitizer cause a rapid activation of the mitogen-activated protein kinases extracellular signal-regulated kinase, p38, and c-Jun NH2 terminal kinase with opposing effects on cell survival. Molecular Cancer Therapy, 7, $1740-1750$.

416. Chan, P. S., Koon, H. K., Wu, Z. G., Wong, R. N. S., Lung, M. L., Chang, C. K., Mak, N. K. (2009). Role of p38 MAPKs in hypericin photodynamic therapy-induced apoptosis of nasopharyngeal carcinoma cells. Photochemistry and Photobiology, 85, 1207-1217.

417. Tang, P., Zhang, D. M., Xuan, N. H., Tsui, S., Waye, M., Kong, S.K., Fong, W. P., Fung, K. P. (2009). Photodynamic therapy inhibits p-glycoprotein mediated multidrug resistance via JNK activation in human hepatocellular carcinoma using the photosensitizer pheophorbide a. Molecular Cancer, 8, 56.

418. Koon, H. K.; Chan, P. S.; Wu, Z. G.; Wong, R. N. S.; Lung, M. L.; Chang, C. K.; Mak, N. K. (2010). Role of mitogen-activated protein kinase in Zn-BC-AM PDT-induced apoptosis in nasopharyngeal carcinoma cells. Cell Biochemistry and Function, 28, 239248.

419. Liu, Y., \& Chang, A. (2008). Heat shock response relieves ER stress. EMBO Journal, 27, 1049-1059.

420. Hetz, C. (2012). The unfolded protein response: controlling cell fate decisions under ER stress and beyond. Nature Reviews Molecular Cell Biology, 13, 89-102.

421. Dai, C., Dai, S., \& Cao, J. (2012). Proteotoxic stress of cancer: implication of the heat-shock response in oncogenesis. Journal of Cellular Physiology, 227, 2982-2987.

422. Helbig, D., Simon, J. C., \& Paasch, U. (2011). Photodynamic therapy and the role of heat shock protein 70 . International Journal of Hyperthermia, 27, 802-810.

423. Ciocca, D. R., \& Calderwood, S. K. (2005). Heat shock proteins in cancer: diagnostic, prognostic, predictive, and treatment implications. Cell Stress \& Chaperones, 10, 86-103. 
424. Mann, M. J., \& Hendershot, L. M. (2006). UPR activation alters chemosensitivity of tumor cells. Cancer Biology and Therapy, 5, 736-740.

425. Åkerfelt, M., Morimoto, R. I., \& Sistonen, L. (2010). Heat shock factors: integrators of cell stress, development and lifespan. Nature Reviews Molecular Cell Biology, 11, 545-555.

426. Park, J., \& Liu, A. Y. C. (2001). JNK phosphorylates the HSF1 transcriptional activation domain: role of JNK in the regulation of the heat shock response. Journal of Biological Chemistry, 82, 326-338.

427. Trinklein, N. D., Chen, W. C., Kingston, R. E., \& M, R. M. (2004). Transcriptional regulation and binding of heat shock factor 1 and heat shock factor 2 to 32 human heat shock genes during thermal stress and differentiation. Cell Stress \& Chaperones, 9, 21-28.

428. Martindale, J. L., \& Holbrook, N. J. (2002). Cellular response to oxidative stress: signaling for suicide and survival. Journal of Cellular Physiology, 192, 1-15.

429. Garrido, C., Brunet, M., Didelot, C., Zermati, Y., Schmitt, E., \& Kroemer, G. (2006). Heat shock proteins 27 and 70: anti-apoptotic proteins with tumorigenic properties. Cell Cycle, 5, 2592-2601.

430. Wang, X., Chen, M., Zhou, J., \& Z, X. (2014). HSP27, 70 and 90, anti-apoptotic proteins, in clinical cancer therapy. International Journal of Oncology, 45, 18-30.

431. Ishikawa, T., Igarashi, T., Hata, K., \& Fujita, T. (1999). C-fos induction by heat, arsenite, and cadmium is mediated by a heat shock element in its promoter. Biochemical and Biophysical Research Communications, 254, 566-571.

432. Sawai, M., Ishikawa, Y., Ota, A., \& Sakurai, H. (2013). The protooncogene JUN is a target of the heat shock transcription factor HSF1. FEBS Journal, 280, 6672-6680.

433. Malhotra, V., \& Wong, H.R. (2002). Interactions between the heat shock response and the nuclear factor- $\mathrm{kB}$ signaling pathway. Critical Care Medicine 30.

434. Saibil, H. (2013). Chaperone machines for protein folding, unfolding and disaggregation. Nature Reviews Molecular Cell Biology, 14, 630-642.

435. Lee, A. H., Iwakoshi, N. N., \& Glimcher, L. H. (2003). XBP-1 regulates a subset of endoplasmic reticulum resident chaperone genes in the unfolded protein response. Molecular and Cellular Biology, 23, 7448-7459.

436. Kincaid, M. M., \& Cooper, A. A. (2007). ERADicate ER stress or die trying. Antioxidants and Redox Signaling, 9, 2373-2387.

437. Kadowaki, H., \& Nishitoh, H. (2013). Signaling pathways from the endoplasmic reticulum and their roles in disease. Genes, 4, 306-333.

438. Yom, S. S., Busch, T. M., Friedberg, J. S., Wileyto, E. P., Smith, D., Glatstein, E., \& Hahn, S. M. (2003). Elevated serum cytokine levels in mesothelioma patients who have undergone pleurectomy or extrapleural pneumonectomy and adjuvant intraoperative photodynamic therapy. Photochemistry and Photobiology, 78, 75-81.

439. Harding, H. P., Zhang, Y., Zeng, H., Novoa, I., Lu, P. D., Calfon, M., Sadri, N., Yun, C., Popko, B., Paules, R., Stojdl, D. F., Bell, J. C., Hettmann, T., Leiden, J. M., \& Ron, D. (2003). An integrated stress response regulates amino acid metabolism and resistance to oxidative stress. Molecular Cell, 11, 619-633.

440. Ye, J., \& Koumenis, C. (2009). ATF4, an ER stress and hypoxiainducible transcription factor and its potential role in hypoxia tolerance and tumorigenesis. Current Molecular Medicine, 9, 411416.

441. He, C. H., Gong, P., Hu, B., Stewart, D., Choi, M. E., Choi, A. M. K., \& Alam, J. (2001). Identification of activating transcription factor 4 (ATF4) as an Nrf2-interacting protein: implication for heme oxygenase-1 gene regulation. Journal of Biological Chemistry, 276, 20858-20865.

442. Gomer, C. J., Ryter, S. W., Ferrario, A., Rucker, N., Wong, S., \& Fisher, A. M. R. (1996). Photodynamic therapy-mediated oxidative stress can induce expression of heat shock proteins. Cancer Research, 56, 2355-2360.

443. Luna, M. C., Ferrario, A., Wong, S., Fisher, A. M. R., \& Gomer, C. J. (2000). Photodynamic therapy-mediated oxidative stress as a molecular switch for the temporal expression of genes ligated to the human heat shock promoter. Cancer Research, 60, 16371644.

444. Hanlon, J. G., Adams, K., Rainbow, A. J., Gupta, R. S., \& Singh, G. (2001). Induction of Hsp60 by photofrin-mediated photodynamic therapy. Journal of Photochemistry and Photobiology B, 64, 55-61.

445. Korbelik, M., Sun, J., \& Cecic, I. (2005). Photodynamic therapy-induced cell surface expression and release of heat shock proteins: relevance for tumor response. Cancer Research, 65, 1018-1026.

446. Mitra, S., Goren, E. M., Frelinger, J. G., \& Foster, T. H. (2003). Activation of heat shock protein 70 promoter with meso-tetrahydroxyphenyl chlorin photodynamic therapy reported by green fluorescent protein in vitro and in vivo. Photochemistry and Photobiology, 78, 615-622.

447. Verrico, A. K., \& Moore, J. V. (1997). Expression of the collagenrelated heat shock protein HSP47 in fibroblasts treated with hyperthermia or photodynamic therapy. British Journal of Cancer, 76, 716-724.

448. Nonaka, M., Ikeda, H., \& Inokuchi, T. (2004). Inhibitory effect of heat shock protein 70 on apoptosis induced by photodynamic therapy in vitro. Photochemistry and Photobiology, 79, 94-98.

449. Verrico, A. K., Haylett, A. K., \& Moore, J. V. (2001). In vivo expression of the collagen-related heat shock protein HSP47, following hyperthermia or photodynamic therapy. Lasers in Medical Science, 16, 192-198.

450. Wang, H. P., Hanlon, J. G., Rainbow, A. J., Espiritu, M., \& Singh, G. (2002). Up-regulation of Hsp27 plays a role in the resistance of human colon carcinoma HT29 cells to photooxidative stress. Photochemistry and Photobiology, 76, 98-104.

451. Ziegler, S. A., Loucks, C., Madsen, S. J., \& Carper, S. W. (2007). Heat shock protein 27 protects against aminolevulinic acidmediated photodynamic therapy-induced apoptosis and necrosis in human breast cancer cells. Journal of Environmental Pathology, Toxicology and Oncology, 26, 173-183.

452. Verfaillie, T., van Vliet, A., Garg, A. D., Dewaele, M., Rubio, N., Gupta, S., de Witte, P., Samali, A., \& Agostinis, P. (2013). Proapoptotic signaling induced by photo-oxidative ER stress is amplified by Noxa, not Bim. Biochemical and Biophysical Research Communications, 438, 500-506.

453. Garg, A. D., Krysko, D. V., Verfaillie, T., Kaczmarek, A., Ferreira, G. B., Marysael, T., Rubio, N., Firczuk, M., Mathieu, C., Roebroek, A. J. M., Annaert, W., Golab, J., de Witte, P., Vandenabeele, P., \& Agostinis, P. (2012). A novel pathway combining calreticulin exposure and ATP secretion in immunogenic cancer cell death. EMBO Journal, 31, 1062-1079.

454. Firczuk, M., Gabrysiak, M., Barankiewicz, J., Domagala, A., Nowis, D., Kujawa, M., Jankowska-Steifer, E., Wachowska, M., Glodkowska-Mrowka, E., Korsak, B., Winiarska, M., \& Golab, J. (2013). GRP78-targeting subtilase cytotoxin sensitizes cancer cells to photodynamic therapy. Cell Death Diseases, 4, e741.

455. Miyata, Y. (2005). Hsp90 inhibitor geldanamycin and its derivatives as novel cancer chemotherapeutic agents. Current Pharmaceutical Design, 11, 1131-1138.

456. Taldone, T., Gozman, A., Maharaj, R., \& Chiosis, G. (2008). Targeting Hsp90: small-molecule inhibitors and their clinical development. Current Opinion in Pharmacology, 8, 370-374.

457. Jego, G., Hazoumé, A., Seigneuric, R., \& Garrido, C. (2013). Targeting heat shock proteins in cancer. Cancer Letters, 332, $275-285$. 
458. Yoon, Y. J., Kim, J. A., Shin, K. D., Shin, D.-S., Han, Y. M., Lee, Y. J., Lee, J. S., Kwon, B.-M., \& Han, D. C. (2011). KRIBB11 inhibits HSP70 synthesis through inhibition of heat shock factor 1 function by impairing the recruitment of positive transcription elongation factor $b$ to the hsp70 promoter. Journal of Biological Chemistry, 286, 1737-1747.
459. Li, Z., Agharkar, P., \& Chen, B. (2013). Therapeutic enhancement of vascular-targeted photodynamic therapy by inhibiting proteasomal function. Cancer Letters, 339, 128-134.

460. Koumenis, C. (2006). ER stress, hypoxia tolerance and tumor progression. Current Molecular Medicine, 6, 55-69. 